\title{
Synthesis and Characterization of ZnO/Graphene Nanostructures for Electronics and Photocatalysis
}

\section{Ebrahim Chalangar}





\title{
Synthesis and Characterization of $\mathrm{ZnO} /$ Graphene Nanostructures for Electronics and Photocatalysis
}

\author{
Ebrahim Chalangar
}

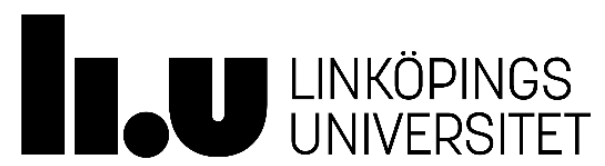

Department of Science and Technology

Division of Physics, Electronics and Mathematics

Linköping University, Sweden

Norrköping 2021 
Cover image:

The cover image shows a cross-sectional SEM image of ZnO nanorods on a CLpatterned rGO/ZnO:Al seed layer on a Si substrate. The different layers are artificially colored for a better perspective.

(C) Ebrahim Chalangar, 2021 (unless otherwise stated)

(c) (i) $($ This work is licensed under the Creative Commons AttributionNonCommercial 4.0 International License. To view a copy of this license, visit http://creativecommons.org/licenses/by-nc/4.0/.

Printed in Sweden by LiU-Tryck, 2021

ISSN: 0345-7524

ISBN: 978-91-7929-682-7 


\section{اين بحر وجود آمده بيرون ز نهفت كس نيست كه اين گَوهر تحقيق بسفت هر كس سخنى از سر سودا گَفتند زآن روى كه هست، كس نمى داند گَفت}

The universe has emerged from hidden depths How? that's a pearl of wisdom no one has pierced

Everyone has conjectured idly,

But none can describe what it really is

Omar Khayyam - 1131 CE 



\section{Abstract}

Recent rapid development of electronics and electro-optical devices demands affordable and reliable materials with enhanced performance. Forming nanocomposites of already well-known materials is one possible route towards novel functional materials with desirable synergistic enhanced properties. Incompatible chemical properties, mismatched crystal structures and weak bonding interactions between the substances, however, often limit the number of possible nanocomposites. Moreover, using an inexpensive, facile, large-area and flexible fabrication technique is crucial to employ the new composites in industrially viable applications.

This thesis focuses on the synthesis and characterization of different zinc oxide/graphene ( $\mathrm{ZnO} / \mathrm{GR}$ ) nanocomposites, well suited for optoelectronics and photocatalysis applications. Two different approaches of i) substrate-free random synthesis, and ii) template-assisted selective area synthesis were studied in detail. In the first approach, $\mathrm{ZnO}$ nanoparticles/rods were grown on GR. The obtained nanocomposites were investigated for better GR dispersity, electrical conductivity and optical properties. Besides, by adding silver iodide to the nanocomposite, an enhanced plasmonic solar-driven photocatalyst was synthesized and analyzed. In the second approach, arrays of single, vertically aligned $\mathrm{ZnO}$ nanorods were synthesized using a colloidal lithography-patterned sol-gel ZnO seed layer. Our demonstrated nanofabrication technique with simple, substrate independent, and large wafer-scale area compatibility improved the alignment and surface density of $\mathrm{ZnO}$ nanorods over large selective growth areas. Eventually, we found a novel method to further enhance the vertical alignment of the $\mathrm{ZnO}$ nanorods by introducing a GR buffer layer between the Si substrate and the $\mathrm{ZnO}$ seed layer, together with the mentioned patterning technique.

The synthesized nanocomposites were analyzed using a large variety of experimental techniques including electron microscopy, photoelectron spectroscopy, $\mathrm{x}$-ray diffraction, photoluminescence and cathodoluminescence spectroscopy for in-depth studies of their morphology, chemical and optical properties. 
Our findings show that the designed $\mathrm{ZnO} / \mathrm{GR}$ nanocomposites with vertically aligned $\mathrm{ZnO}$ nanorods of high crystalline quality, synthesized with the developed low-cost nanofabrication technique, can lead to novel devices offering higher performance at a significantly lower fabrication cost.

Keywords: zinc oxide, graphene, nanostructure, nanocomposite, conjugated electronics, photocatalysis, nanofabrication, colloidal lithography, chemical bath deposition, sol-gel 


\section{Populärvetenskaplig sammanfattning}

I dag introduceras ny teknik i våra liv i en allt snabbare takt. Nya elektroniska produkter förväntas underlätta vår vardag genom att göra saker snabbare, mer exakt, säkrare och billigare. Utvecklingen av nya inbäddade teknologier som Internet-of-things (IoT), 5G-kommunikation, artificiell intelligens och maskininlärning vilar tungt på utvecklingen av ny hårdvara, som i sin tur förutsätter tillgång till nya avancerade material med förbättrade elektroniska egenskaper och ett konkurrensmässigt pris.

Att utveckla nya funktionella material med önskvärda egenskaper är en komplicerad process som involverar olika grenar inom naturvetenskapen, inklusive fysik, kemi och materialvetenskap. Att koppla samman etablerad halvledarindustri med nanoteknik är ett utmärkt exempel på detta partnerskap mellan olika vetenskapsområden för bättre materialinnovation. I denna process skapas ofta nya funktionella material med önskvärda förbättrade egenskaper genom att integrera konventionella halvledarmaterial med varandra på nya innovativa sätt. Även om detta kan tyckas enkelt i teorin, begränsas utvecklingen i praktiken ofta av utmaningar som till exempel oförenliga kemiska egenskaper, olika kristallstrukturer eller inkompatibla elektriska bindningar mellan ämnena antalet möjliga kombinationer. Dessutom är det viktigt att utveckla nya enkla, billiga och flexibla tillverkningsmetoder för storskalig produktion av dessa nya kompositmaterial.

Den här avhandlingen fokuserar på utveckling av nya funktionella nanokompositer, bestående av halvledarmaterialet zinkoxid ( $\mathrm{ZnO})$ och det två-dimensionella materialet grafen, för tillämpningar inom optoelektronik och fotokatalys. Zinkoxid kan odlas som tunna nålliknande strukturer som kallas nanostavar med en billig lösningsbaserad teknik. Tack vare sina utmärkta elektriska och optiska egenskaper har $\mathrm{ZnO}$ använts flitigt i många applikationer som till exempel lysdioder, elektrokemiska sensorer och gassensorer. Grafen har utmärkt sig som ett fantastiskt material med helt unika elektriska, optiska och mekaniska egenskaper som väsentligt förbättrar den optoelektroniska och fotokatalytiska effektiviteten hos $\mathrm{ZnO}$ /grafen nanokompositer. 
Jag har i mitt arbete studerat elektrisk ledningsförmåga, optiska egenskaper och fotokatalyseffektivitet hos våra designade $\mathrm{ZnO} /$ grafen nanokompositer under simulerad solstrålning. Dessutom har jag odlat vertikalt ordnade ZnO nanostavar i små nanohål på mönstrade substrat belagda med ett tunt lager av antingen $\mathrm{ZnO}$, eller med ett sandwichlager bestående av $\mathrm{ZnO}$ och grafen med hjälp av en ny billig metod som kallas kolloidal litografi. Denna tillverkningsmetod erbjuder enkel odling av högkvalitativa (kristallina) ZnO nanostavar med god kontroll över ytdensiteten på stora godtyckliga materialytor. Våra resultat visar att dessa nya högkvalitativa $\mathrm{ZnO} /$ grafen nanokompositer potentiellt erbjuder en ny materialplattform för tillverkning av exempelvis billig högpresterande optoelektronik. 


\section{Acknowledgment}

The past five years were the most challenging but exciting part of my life. Many people helped me during this journey, although only one name appears on the cover of this thesis. I would like to express my sincere gratitude to all of you.

My main supervisor Håkan Pettersson for giving me the opportunity and freedom to work on this project. Not only did I learn scientific matters from you, but also your precision, tolerance and respectful behavior will be my intentions. I always felt comfortable working with you during these years, oh wait...! maybe I should exclude the deadline times.

My co-supervisors, Magnus Willander and Omer Nour, for all your supports, encouragement, fruitful discussions, and always accessible communication. Also, thanks go to the lab crew, Lars Gustavsson, Thomas Karlsson and Meysam Karami, for keeping the lab running.

I also would like to thank my colleagues at Halmstad University. Emil Nilsson, who taught me the very basics of RF components and measurements, and also for assisting me on the first days after I arrived in Sweden. Pererik Andreasson for all his support and always pleasant discussions. Jessika Rosenberg and Stefan Gunnarsson for all their help with administrative issues. I especially thank Struan Gray for revising this thesis.

Also, I appreciate all my former and current friends at Halmstad University and Linköping University.

Being a PhD student not only shaped my life but also affected my beloveds' lives. With my deepest gratitude, I wish the best for my mother and my father, who endured the distance to us and their grandchildren during these years, and for my wife for her endless love and kind support.

Ebrahim Chalangar,

Linköping, March 2021 


\section{List of publications}

\section{Papers included in this thesis:}

Paper I.

Influence of Morphology on Electrical and Optical Properties of Graphene/Al-Doped ZnO-Nanorod Composites

Ebrahim Chalangar, Houssaine Machhadani, Seung-Hyuk Lim, K. Fredrik Karlsson, Omer Nur, Magnus Willander, and Håkan Pettersson.

Nanotechnology, 2018. https://doi.org/10.1088/1361-6528/aad3ec.

Contribution: I prepared and characterized the samples and wrote the first draft. I was actively involved in conceiving the research idea and analyzing the results.

Paper II.

\section{Graphene-Based Plasmonic Nanocomposites for Highly Enhanced Solar-Driven Photocatalytic Activities}

Rania E. Adam ${ }^{\ddagger}$, Ebrahim Chalangar ${ }^{\ddagger}$, Mahsa Pirhashemi, Galia Pozina, Xianjie Liu, Justinas Palisaitis, Håkan Pettersson, Magnus Willander, and Omer Nur. RSC Advances, 2019. https://doi.org/10.1039/C9RA06273D. ( Both co-first author)

Contribution: I took part in material synthesizing and performed the electron microscopy analysis. I contributed to analyzing the data, writing the first draft and revising the final manuscript.

Paper III.

Synthesis of Vertically Aligned ZnO Nanorods Using Sol-Gel Seeding and Colloidal Lithography Patterning

Ebrahim Chalangar, Omer Nur, Magnus Willander, Anders Gustafsson, and Håkan Pettersson.

Nanoscale Research Letters, 2021. https://doi.org/10.1186/s11671-02103500-7.

Contribution: I prepared and characterized the samples and wrote the first draft. I was actively involved in conceiving the research idea, analyzing the results, and revising the final manuscript. 
Paper IV.

Nanopatterned reduced graphene oxide/Al-doped $\mathrm{ZnO}$ seed layer for vertical growth of single $\mathrm{ZnO}$ nanorods on various substrates

Ebrahim Chalangar, Elfatih Mustafa, Omer Nur, Magnus Willander, Anders Gustafsson and Håkan Pettersson. In manuscript

Contribution: I prepared and characterized the samples and wrote the first draft. I was actively involved in conceiving the research idea, analyzing the results, and revising the final manuscript.

\section{Papers not included in this thesis:}

Efficient Photo Catalysts Based on Silver Doped ZnO Nanorods for the Photo Degradation of Methyl Orange

Muhammad Ali Bhatti, Aqeel Ahmed Shah, Khalida Faryal Almani, Aneela Tahira, Seyed Ebrahim Chalangar, Ali dad Chandio, Omer Nur, Magnus Willander, and Zafar Hussain Ibupoto.

Ceramics International, 2019. https://doi.org/10.1016/j.ceramint.2019.08.027.

Contribution: I was involved in sample preparation for electron microscopy, performed the SEM and EDS measurements and read the final manuscript.

Facile Synthesis of Copper Doped ZnO Nanorods for the Efficient Photo Degradation of Methylene Blue and Methyl Orange

Aqeel Ahmed Shah, Muhammad Ali Bhatti, Aneela Tahira, Ali Dad Chandio, Iftikhar A. Channa, Ali Ghulam Sahito, Ebrahim Chalangar, Magnus Willander, Omer Nur, and Zafar Hussain Ibupoto.

Ceramics International, 2020. https://doi.org/10.1016/j.ceramint.2019.12.024.

Contribution: I was involved in sample preparation for electron microscopy, performed the SEM and EDS measurements and read the final manuscript. 



\section{Table of Contents}

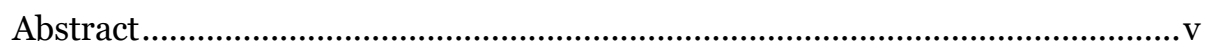

Populärvetenskaplig sammanfattning ...................................................... vii

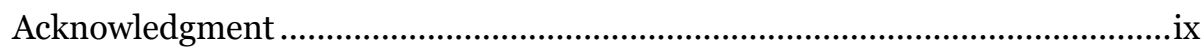

List of publications .........................................................................................

Table of Contents............................................................................................... xii

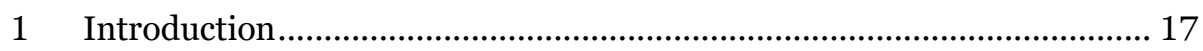

$1.1 \quad$ Zinc oxide/graphene nanocomposites ............................................. 18

1.2 Zinc oxide/graphene heterostructures............................................. 20

$1.3 \quad$ Thesis Aim and Outline ........................................................... 21

2 Materials background ............................................................................ 23

2.1 Semiconductors and electronic band structure ............................... 23

$2.2 \quad$ Zinc oxide.................................................................................. 24

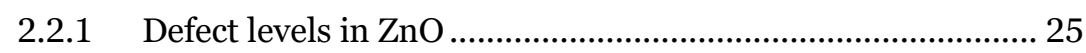

2.2.2 Doping of $\mathrm{ZnO}$.................................................................... 26

2.2.3 Polarity in $\mathrm{ZnO}$ nanorods ..................................................... 27

2.2.4 Surface defects and band bending in $\mathrm{ZnO}$ nanorods .............. 28

$2.3 \quad$ Graphene and graphene oxide......................................................... 29

$2.4 \quad \mathrm{ZnO}$-graphene heterojunctions ....................................................... 31

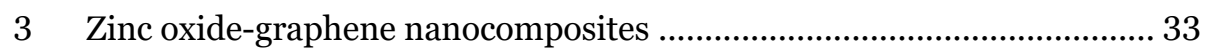

3.1 Synthesis method......................................................................... 33

3.1.1 Chemical bath deposition of $\mathrm{ZnO}$ nanorods............................ 34

3.1.2 ZnO nanoparticles growth on GR nanoplates ........................ 36

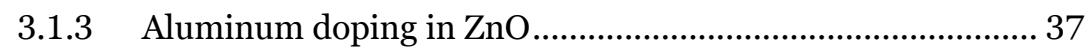

3.1.4 Adding silver iodide into $\mathrm{ZnO} / \mathrm{GR}$ nanocomposites ............... 38

$3.2 \quad$ Characterization methods ............................................................. 39 


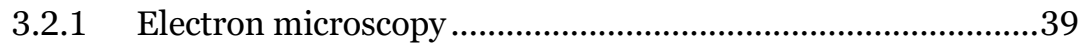

3.2.2 UV-Vis absorption spectroscopy ………………….................40

3.2.3 Photoluminescence spectroscopy (PL) ……………………......41

3.2.4 Cathodoluminescence spectroscopy ……………………........42

3.2.5 X-ray diffraction analysis (XRD)...........................................

3.2.6 X-ray photoelectron spectroscopy (XPS) ..................................42

3.2.7 Surface resistivity measurements ............................................43

3.2.8 Photoconductivity measurements.............................................4

3.2.9 Photodegradation efficiency measurements............................45

3.3 ZnO-NRs/GR for electronics applications .........................................46

3.3.1 Effects of $\mathrm{pH}$ on the morphology of ZnO-NRs/GR..................46

3.3.2 Improving the conductivity of ZnO-NRs/GR by Al-doping ...47

3.3.3 Bonding quality between GR and ZnO-NRs ...........................48

3.3.4 Optical properties of $\mathrm{ZnO} / \mathrm{GR}$ nanocomposites .....................49

3.4 ZnO-NPs/GR for photocatalytic applications....................................52

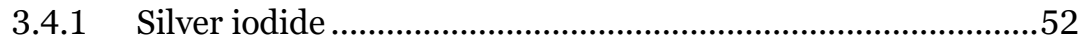

3.4.2 Characterization of $\mathrm{ZnO} / \mathrm{GR} / \mathrm{Ag} / \mathrm{AgI}$ nanocomposites ...........53

3.4.3 Photocatalytic performance of the nanocomposites.................55

4 Template-assisted nanofabrication of ZnO-NRs/GR ..................................57

4.1 Synthesis methods .........................................................................

4.1.1 Synthesis of GO using the improved Hummer's method ........58

4.1.2 ZnO sol-gel solutions ..............................................................60

4.1.3 CBD of ZnO-NRs on patterned substrates ...............................60

4.2 Nanofabrication of patterned substrates ..........................................61

4.2.1 Spray coating of GO buffer layers..............................................62

4.2.2 Dip-coating of the $\mathrm{ZnO}$ sol-gel solution ..................................64

4.2.3 Colloidal lithography (CL) patterning.....................................64

4.2.4 Thermal evaporation ..............................................................65

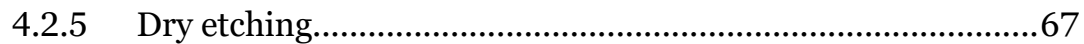

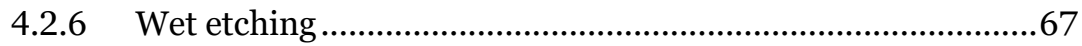

4.3 Characterization methods - Atomic force microscopy ....................68

4.4 Characterization of the CL-patterned substrates.............................70 
4.4.1 Why using $\mathrm{Si} / \mathrm{GO}$ substrates? .................................................. 70

4.4.2 Improved $\mathrm{ZnO}$ seed layers by dip-coating............................... 71

4.4.3 Reduced GO/Al-doped $\mathrm{ZnO}$ seed layers.................................. 73

4.4.4 Distribution of polystyrene nanobeads on surfaces................ 73

5 Conclusions and Outlook....................................................................... 77

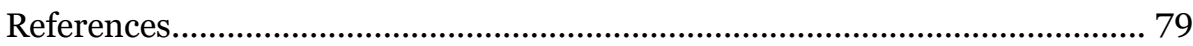

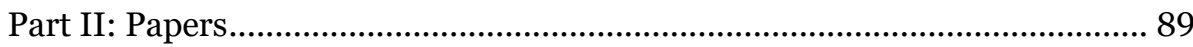





\section{Introduction}

Today, electronic devices are evolving very rapidly, with greater functionality and faster and more reliable operations. This progress has affected our daily lives and led to further integration of the latest technologies into various aspects of our lives. The Internet-of-things (IoT), comprised of novel embedded systems with a massive number of collective sensors, is now being marketed to assist with health, education, transportation, and many more. 5G-communication, with its remarkable predicted advantages, provides the faster, ultra-reliable, and massive wireless connection between devices that is demanded by other applications. In step with the expansion of these technologies, the need for more energy, and the consequent increase in environmental pollution, become more pronounced. So, the future of sustainable growth relies on developing more efficient hardware to balance requirements with resources. To improve devices' efficiency, additional new functional materials are required. The new devices should also be affordable and inexpensive to facilitate widespread adoption of new massively embedded applications.

Despite all efforts, the range of materials with the desired electrical and optical properties is still limited by their functionality or cost. One way to overcome these challenges is to develop new composites of already existing, well-known materials with desirable synergistic enhanced properties. In fact, recent advances in nanotechnology have provided more insights towards a better understanding and implementation of novel composite materials in various applications. This thesis particularly deals with semiconductor materials with nanometer-scale dimensions and their composition with other nano-electronic materials. 
Research into innovative nanocomposite materials and their new applications has attracted much recent interest and effort. A vast number of metal-semiconductor [1], multiple metal oxides [2], and organic-inorganic [3] nanocomposites with improved properties have been generated and used in broad fields of applications such as energy, hydrogen evolution, the environment, disinfection and purification. A combination of semiconductors and 2D materials such as graphene (GR) is another exciting class of composite materials with promising characteristics for photocatalysis, sensors, energy, and electronic applications [4]. To date, various such nanocomposites, including group IV (Si), group II-VI ( $\mathrm{ZnO}, \mathrm{ZnS}$ ), group III-V (GaAs, GaN), and metal oxide semiconductors ( $\left.\mathrm{TiO}_{2}, \mathrm{ITO}\right)$, have been realized [4].

The research presented in this thesis is mainly focused on the possible composites of zinc oxide $(\mathrm{ZnO})$ and $\mathrm{GR}$, exploring their fundamental properties and realizing their potential applications in electronics and photocatalysis. The materials were selected based on their suitable intrinsic optical and electrical properties for the mentioned applications. Individually, these two well-known materials have been thoroughly investigated in many previous studies. But the combination of the two has recently gained popularity due to the resulting synergistic enhanced properties, which are not available in each of the single materials.

\subsection{Zinc oxide/graphene nanocomposites}

Composites of $\mathrm{ZnO}$ and GR have been realized in several research reports [5-8] with strong evidence of a good crystal growth compatibility due to excellent matching of their crystal lattices [9-11]. Growth of various $\mathrm{ZnO}$ structures on a GR surface in the forms of nanoparticles (OD) and nanorods (1D) can be found in the literature, prepared by various higher-temperature, vacuum-required, or solution-based growth techniques. Also, a wide range of applications of these composite materials in electronics, optoelectronics, photovoltaics, and sensors have been demonstrated [4]. Despite these efforts, a research gap between the optimal functionality and the fabrication simplicity of $\mathrm{ZnO} / \mathrm{GR}$ is still observable. In other words, in the majority of the reports, production of high- 
performance $\mathrm{ZnO} / \mathrm{GR}$ nanostructures usually required sophisticated methods, leading to more expensive products. Further developments in the nanocomposite's growth procedure with significant control over the final structure are crucially needed to meet the criteria of optimal desired properties and low-cost production. This is what motived us to conduct this research work.

This PhD study, therefore, probes ways to fill this gap by combining a simple growth technique and precisely designing the $\mathrm{ZnO} / \mathrm{GR}$ structures suited for electronics or as a photocatalyst. Growth of different morphologies of $\mathrm{ZnO}$ on the GR nanoplates was achieved, and the nanocomposites were investigated for higher electrical conductivity, optical properties, and enhanced photocatalytic efficiency. Moreover, we pursued a method to fully control the final structures of the nanocomposites with respect to their density, alignment, ordering, and feature size, using colloidal lithography and optimized seed layers.

Our results show successful growth of Al-doped $\mathrm{ZnO}$ nanorods (NRs) on GR nanoplatelets with optimized porosity and electrical conductivity, suitable for electronic applications in which inexpensive large-volume of conductive materials are required, e.g., 3D printing. Also, highly efficient solar-driven photocatalysts of $\mathrm{ZnO}$, GR and silver iodide (AgI) for removing organic pollutants from water were achieved and exhibited. Additionally, a novel nanofabrication method of vertically aligned $\mathrm{ZnO}$ nanorods on different substrates, using a combination of colloidal lithography and sol-gel seeded layers, was demonstrated.

Our findings are important for further understanding the basic charge carrier transport mechanism in the semiconductor-graphene interfaces, photocarrier generation and recombination, and energy defect levels in the $\mathrm{ZnO} / \mathrm{GR}$ nanocomposites. The results can be used to tailor the conductivity of future 3Dprintable material with large volumes and inexpensive porous structures. Also, the solar-driven photocatalysts developed here are beneficial for environmental purification since they can remove organic pollutants from water using the energy in sunlight. Moreover, our nanofabrication method offers a simple and inexpensive solution for controlling the NRs' growth and enhancing their alignment with substrate-independent flexibility. Finally, our work can help to produce cheaper, porous, conjugated graphene-semiconductor composites to 
fabricate high-performance devices where vertically ordered $\mathrm{ZnO}$-NRs of high crystalline quality are essential.

\subsection{Zinc oxide/graphene heterostructures}

Similar to conventional metal-semiconductor junctions, GR-semiconductor junctions generally show rectifying characteristics [12]. When two materials are atomically close to each other, a junction is formed at their interface. The type of junction depends on the similarity of the crystal structure, e.g., bandgap difference, valence band matching, lattice mismatch, and the type and level of doping in the two materials. If the materials are the same, a homojunction is formed at the interface, for example, a p-n junction of a semiconductor with different donor and acceptor dopants.

A heterojunction forms at the interface of two dissimilar semiconductor materials, usually by heteroepitaxial growth of fairly lattice-matched semiconductors on top of each other. The crystal lattice mismatch is the limiting parameter for the epitaxial growth, inducing atomic displacements (strain) and dislocations at the interface unless the epitaxial layer is extremely thin. As a result of the difference in bandgap energy and the band alignments at the interface, such heterojunctions are the building-blocks for many interesting applications, including laser diodes, light-emitting diodes (LEDs), resonant tunneling diodes, photodetectors, and solar cells.

The lattice mismatch between the joint materials can be tolerated if lowerdimensional materials, e.g., nanoparticles (0D) or nanorods (1D), with a lower interfacial area, are used. In this case, the strain energy at the heterojunction is relieved via elastic relaxations, leading to more stable structures [13, 14]. The stability of the structure depends on the lattice mismatch factor and the size of the interfacial area, or equivalently, the diameter of the grown particles. Fig. 1-1 shows a typical stability diagram for a heterostructure nanorod system with two stable and unstable regions, depending on the NRs' diameter and the lattice mismatch factor. The nanoparticle and nanorod morphologies of $\mathrm{ZnO}$ in our developed nanocomposites were chosen based on this consideration. 


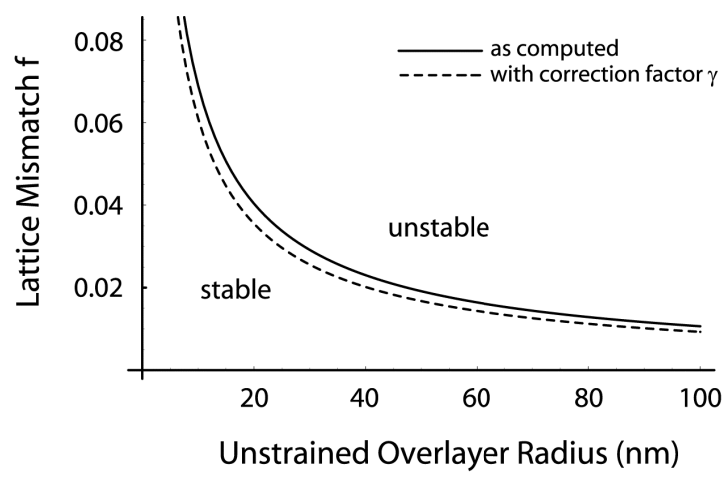

Fig. 1-1. Stability diagram for a heterostructure nanorod system with respect to the formation of misfit dislocations. Reprinted by permission from Springer Nature: MRS Online Proceedings Library [13].

\subsection{Thesis Aim and Outline}

This thesis mainly focuses on a two-material system of $\mathrm{ZnO}$ and GR nanocomposites. Two different approaches for synthesizing the $\mathrm{ZnO} / \mathrm{GR}$ nanocomposites were employed. In the first approach, substrate-free random $\mathrm{ZnO} / \mathrm{GR}$ structures were synthesized and optimized for electronics and photocatalysis applications. Paper I covers the most important results for ZnO-NRs/GR nanocomposites suited to electronics application, including the effect of the growth parameters on the NRs' morphologies; Al-doping impact on the final conductivity; optical properties; and photocarrier generation and recombination in the nanocomposites.

In Paper II, various composites of $\mathrm{ZnO}$ nanoparticles, GR and AgI, with a broader absorption peak in the visible range, were fabricated and tested for higher photocatalytic efficiency. The prepared nanocomposites were characterized using various experimental techniques and were employed to remove organic pollutants from water.

In the second approach, we attempted to improve the order and alignment of the ZnO-NRs, by first appropriately seeding a Si substrate and then patterning the selective area using colloidal lithography (CL). Paper III demonstrates 
the patterning technique and the seed layer preparation method used later in Paper IV to fabricate the GR/ZnO-NRs vertically aligned nanostructures.

The thesis places all the experimental achievements in a united context by providing background information in Chapter 2, along with more details about the experiments and the analytical techniques employed. Chapter III deals with Papers I and II, describing the use of $\mathrm{ZnO} /$ GR nanocomposites as a conductive, printable material and a photocatalyst. Chapter IV introduces the CL-patterning technique and summarizes the work reported in Papers III and IV. Finally, my $\mathrm{PhD}$ research is concluded in Chapter $\mathrm{V}$, followed by a discussion of future challenges and outlooks. 


\section{Materials background}

Before going into our nanocomposite synthesis process in detail, an introduction to the basic concepts of semiconductors, including heterostructures, energy levels, defects, and doping, is given here. This chapter aims to familiarize the reader with the concepts that will be used later, and summarizes what I learned during my experimental research.

\subsection{Semiconductors and electronic band structure}

Although conductors conduct electrical currents very well, semiconductors are the most exciting materials in electronics. The difference between conductors and semiconductors comes from their atomic structure and the electronic configuration in their crystal lattices. Atoms of an element can form chemical bonds with each other and arrange themselves in a periodic crystal lattice by reforming their electron orbitals to a hybrid orbital configuration. The periodic atom positions introduce a periodic electric potential in the crystal lattice. By applying Bloch's theorem and solving Schrödinger's equation in the tight-binding model, including spin-orbit coupling, the material's electronic band structure can be derived. Detailed equations are to be found in most solid-state textbooks, and only the most important results are given here.

The electronic band structure of a crystalline solid is determined by the density of states in its different energy levels, including the valence band $\left(\mathrm{E}_{\mathrm{v}}\right)$, conduction band $\left(\mathrm{E}_{\mathrm{c}}\right)$, and the bandgap $\left(\mathrm{E}_{\mathrm{g}}\right)$, which has zero density of states. The electrons fill the available energy levels from lower to higher energies according to the Pauli principle, which results in the well-known Fermi-Dirac distribution function (Eq. (2.1)) at a given temperature (T), where $k_{\mathrm{B}}$ is the Boltzmann's constant and $\mathrm{E}_{\mathrm{F}}$ is the Fermi energy level. 


$$
F(E)=\frac{1}{1+e^{\left(E-E_{F}\right) / k_{B} T}}
$$

$\mathrm{E}_{\mathrm{F}}$ is defined as the energy level with a $50 \%$ probability of occupation under conditions of thermodynamic equilibrium. Equivalently, $\mathrm{E}_{\mathrm{F}}$ is the total chemical potential of the electrons, which is equal to the energy required to add or remove an electron into the system. EF can be measured directly by a voltmeter relative to a reference point. EF changes result from any variation in the kinetic or potential energy of the electrons, such as from an external potential, adding acceptor or donor impurities to semiconductors, or many kinds of mechanical, photonic, or thermal excitation in materials. Fig. 2-1 shows the typical $\mathrm{E}_{\mathrm{F}}$ position in various types of materials relative to their valence and conduction bands.

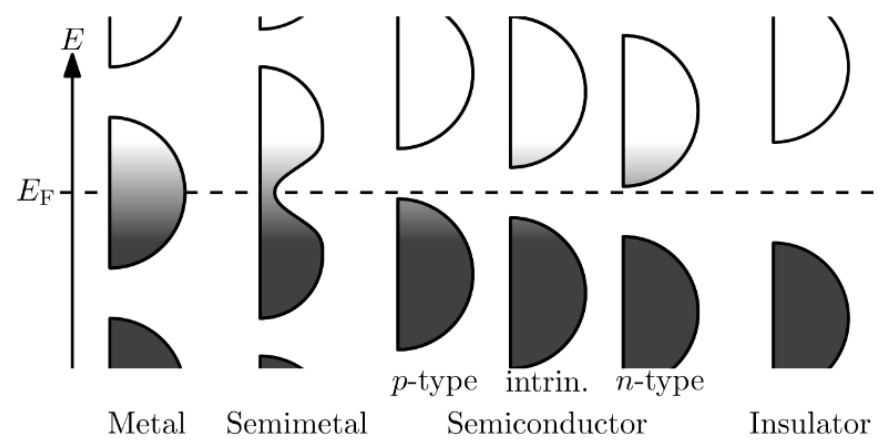

Fig. 2-1. Energy band gap diagram for various materials. The horizontal and vertical axes indicate the density of states and the energy levels, respectively. The black-white contrast shows the Fermi-Dirac distribution of electrons. From Wikipedia under CC0 1.0 License.

\subsection{Zinc oxide}

Zinc oxide has been studied for decades as a well-characterized, unintentionally n-type semiconductor. It has a hexagonal wurtzite crystal structure and a wide direct bandgap of 3.2-3.4 eV at room temperature. Because of its high mechanical and thermal stability, large exciton binding energy of $60 \mathrm{meV}$, and promising electrical and optical properties [15], it has attracted a great deal of interest in a broad range of applications, including LEDs, sensors, piezoelectrics, and electrochemistry [16]. The other beneficial aspect of using $\mathrm{ZnO}$ is the excellent 
flexibility in growth techniques and the various possible morphologies of nanoparticles (NPs), NRs and thin films. In the following section, the defects and dopants in a $\mathrm{ZnO}$ crystal, which are the main origin of its electrical and optical characteristics, are briefly reviewed.

\subsubsection{Defect levels in $\mathrm{ZnO}$}

During the $\mathrm{ZnO}$ growth process, various defects may be introduced in the crystal lattice, with corresponding defect energy levels in the $\mathrm{ZnO}$ band structure. Donor-like defects of oxygen vacancy $\left(\mathrm{V}_{0}\right)$ and interstitial zinc $\left(\mathrm{Zn}_{\mathrm{i}}\right)$, and acceptorlike defects of zinc vacancy $\left(\mathrm{VZn}_{\mathrm{Zn}}\right)$ and interstitial oxygen $\left(\mathrm{O}_{\mathrm{i}}\right)$ are the most likely native point defects in $\mathrm{ZnO}$.

In many publications, the unintentionally n-type conductivity of $\mathrm{ZnO}$ has been attributed to the $\mathrm{V}_{\mathrm{O}}$ and $\mathrm{Zn}_{\mathrm{i}}$, but more accurate studies have shown that these point defects cannot be the cause of the n-type behavior, as neither engaged in the conductivity [17]. The reason is that the donor-like native defects in $\mathrm{ZnO}$ are either deep level donors or have a high formation energy and so a very low formation probability [17-19]. In general, only the shallow defect levels, close to the band edges, can be thermally ionized at room temperature and participate in the conductivity, and not the deep levels [19]. Fig. 2-2 shows the energy levels of the native defects in the $\mathrm{ZnO}$ bandgap in comparison to $\mathrm{H}$ and $\mathrm{Al}$ donor levels. Although $\mathrm{V}_{\mathrm{O}^{2+}}$ and $\mathrm{Zn}_{\mathrm{i}}$ shallow defects are relatively close to the $\mathrm{CB}$, they are unlikely to form due to their high formation energy, showing very low concentrations in the $\mathrm{ZnO}$ band structure under conditions of thermodynamic equilibrium [19]. These defect levels are the origin of the visible emissions in the luminescence spectra caused by electron-hole recombination.

Instead, the interstitial hydrogen $\left(\mathrm{H}^{+}\right)$impurity has been proposed as a shallow donor and the source of the $\mathrm{n}$-type conductivity of $\mathrm{ZnO}$ at room temperature. Hydrogen impurity atoms can bond to an $\mathrm{O}$ atom, forming an - $\mathrm{OH}$ group, or replace $\mathrm{O}$ in $\mathrm{ZnO}$ and form four equal bounds to four neighboring $\mathrm{Zn}$ atoms with relatively low formation energies. The unintentional presence of $\mathrm{H}$ in $\mathrm{ZnO}$ comes from the available $\mathrm{H}$ in the growth precursors. It can be enhanced by 
annealing the grown $\mathrm{ZnO}$ in an $\mathrm{H}_{2}$ atmosphere, resulting in higher electrical conductivity.

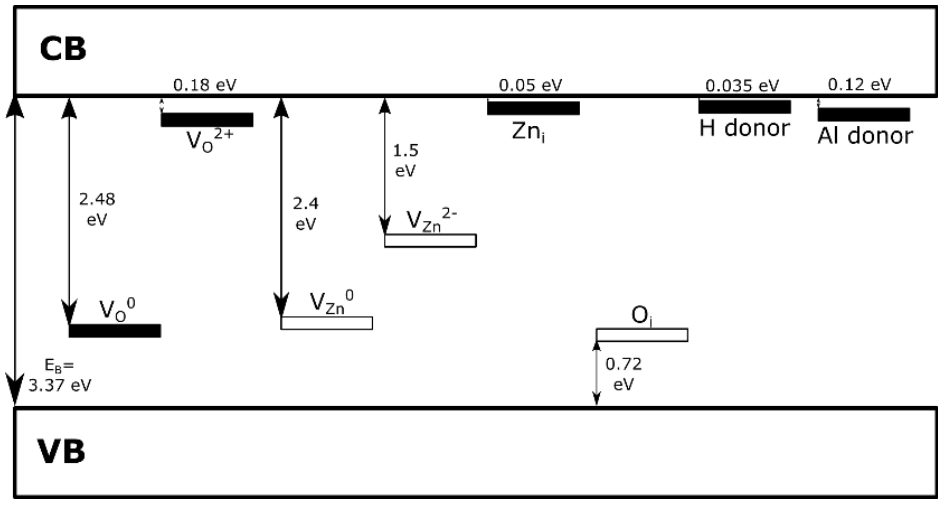

Fig. 2-2. Energy band diagram of native defects and $\mathrm{H}$ and $\mathrm{Al}$ donor impurities in $\mathrm{ZnO}$. Filled and empty bars represent donor and acceptor levels, respectively. Gathered from Refs. [17-21].

\subsubsection{Doping of $\mathrm{ZnO}$}

In addition to the unintentional native defects and $\mathrm{H}$ impurity, $\mathrm{ZnO}$ can be intentionally doped by group-13 impurities of $\mathrm{B}, \mathrm{Al}, \mathrm{Ga}$ and In, or by $\mathrm{F}$ [22]. Among these n-type dopants, $\mathrm{Al}$, with its low formation energy and relatively low ionization energy of $120 \mathrm{meV}$ (Fig. 2-2), is a promising candidate, particularly in solution-based $\mathrm{ZnO}$ growth techniques. High levels of $\mathrm{Al}$-doping, up to the degenerate level with a carrier concentration of $10^{20} \mathrm{~cm}^{-3}$, have been achieved, changing the electrical properties of $\mathrm{ZnO}$ from an insulator to a metal. In contrast to the success achieved with n-type material, p-type doping of $\mathrm{ZnO}$ is notably difficult and still challenging.

As a potential application, $\mathrm{Al}$-doped $\mathrm{ZnO}$ (AZO) has been widely studied as a transparent conductive oxide (TCO) and is presently considered for substituting conventional TCOs on the market with $85 \%$ optical transmittance in the visible range and electrical resistivity down to $10^{-3} \Omega . \mathrm{cm}$ [23-25]. A comparison of different TCOs, including AZO, can be found in Ref. [26]. Usually, post-treatments of thermal or UV annealing are required to improve the conductivity of the final Al-doped $\mathrm{ZnO}$ structures. 
The bandgap of $\mathrm{ZnO}$ can be changed corresponding to the doping level and the carrier concentration. The shift of the bandgap is governed by the contribution of two opposite phenomena, which usually compensate each other: increasing the bandgap proportional to the carrier density (Burstein-Moss effect) and bandgap narrowing [27].

\subsubsection{Polarity in ZnO nanorods}

Hexagonal ZnO-NRs exhibit an electric dipolar moment along the c-axis and six non-polar m-plane sidewalls in [1100] directions with relatively low free surface energy. The polarity direction is defined as the direction of a vector from the positively-charged Zn-polar to the negatively-charged O-polar face terminations at each unit cell (Fig. 2-3). The Zn- and O-polar faces, with various chemical reactivities, adsorb ambient $\mathrm{OH}^{-}$hydroxyl groups and $\mathrm{H}^{+}$ions, respectively, minimizing their free energies and forming a double electrical layer on the surface [28].

\section{Wurtzite ZnO}

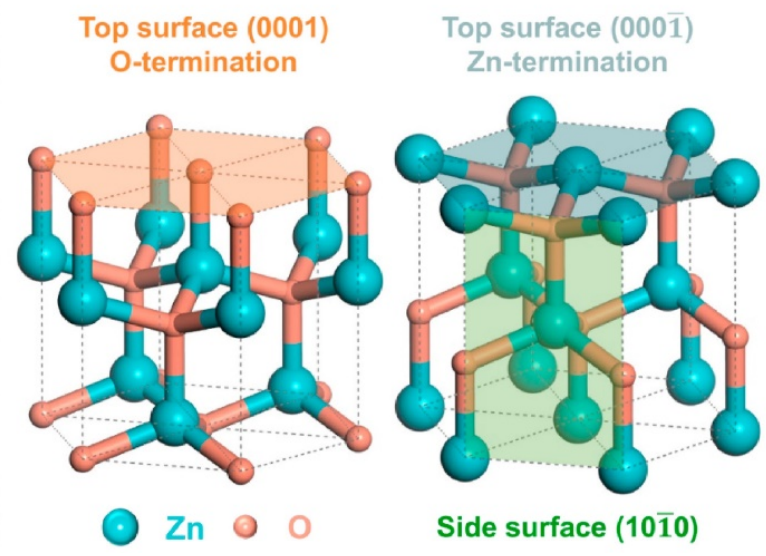

Fig. 2-3. O-polar and Zn-polar faces along the c-axis with flipped-over polarities. Reprinted from [29], Copyright 2020 with permission from Elsevier.

Growing of ZnO-NRs on non-epitaxial substrates is usually required a precoated optimum nuclei (so-called seed layer) on the surface. The polarity of the seed layer is transferred up into the NRs by a layer-by-layer homo-epitaxial growth 
of the O- and Zn-polar unit cells on the bottom polar faces. In fact, the polar cplane top front is the driving force for the anisotropic one-dimensional growth of the ZnO-NRs [28]. Thus, any induced surface charge or applied normal-tosubstrate electric field will facilitate the polar alignment and can lead to higher NRs alignment [30]. Fig. 2-4 shows the polarity transfer from the seed layer into the grown $\mathrm{ZnO}$ nanorods.

The polarity in ZnO-NRs also has a vast influence on the native defects' spatial distribution within the NRs. It has been shown that the Vo defects have a much smaller formation energy on the non-polar m-plane surfaces, which will lead to higher Vo concentration on the surface of ZnO-NRs [29]. The high surface defect concentration has an important effect on persistent photoconductivity in $\mathrm{ZnO}$, which is explained below.

(a)

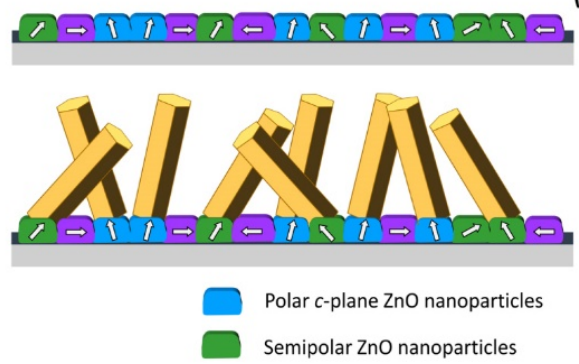

(b)

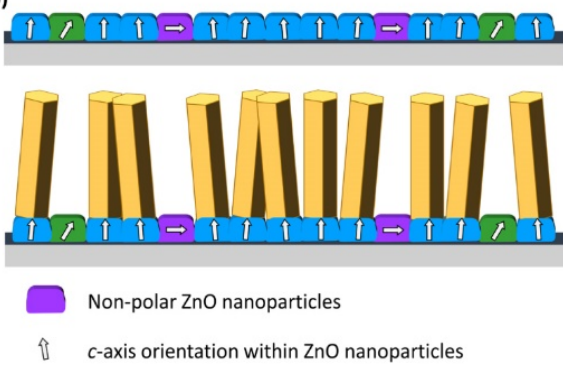

Fig. 2-4. Effect of a (a) poorly and a (b) highly polar oriented polycrystalline seed layer on the growth of $\mathrm{ZnO}$-NRs. Reproduced with permission from [31]. (C) IOP Publishing. All rights reserved.

\subsubsection{Surface defects and band bending in $\mathrm{ZnO}$ nanorods}

Due to the high concentration of $\mathrm{V}_{0}$ defects on the $\mathrm{ZnO}$ surface, oxygen molecules in the atmosphere are attracted and chemisorbed on the $\mathrm{ZnO}$ surface with a thermal energy barrier of about $0.25 \mathrm{eV}$ [32]. The surface chemisorbed $\mathrm{O}_{2}$ molecules attract and capture free electrons from the $\mathrm{ZnO}$ core. Due to the low concentration of free electrons in undoped samples, the electric field inside the semiconductor, induced by the surface chemisorbed ionized $\mathrm{O}_{2}^{-}$, cannot be effectively screened. In this scenario, a built-in electric field is formed near the surface, and the energy bands are bent. Consequently, the area is depleted of free 
carriers, similar to a p-n junction [33-36]. Fig. 2-5 shows schematically band bending on the surface of a $\mathrm{ZnO}-\mathrm{NR}$ and the free carrier depletion shell.

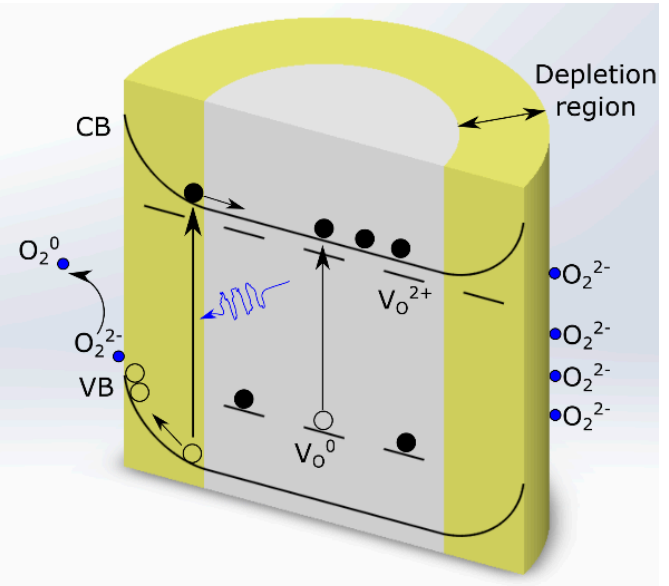

Fig. 2-5. Energy band bending on a $\mathrm{ZnO}-\mathrm{NR}$ surface due to the surface chemisorbed $\mathrm{O}_{2}$. The yellow area indicates the depletion region.

Usually, UV exposure increases the conductivity of $\mathrm{ZnO}$ by oxidizing the chemisorbed species, e.g., oxygen or water, on the $\mathrm{ZnO}$ surface. Under UV irradiation, the photogenerated holes migrate toward the surface and are consumed by the surface ionized $\mathrm{O}_{2}$ species, leaving behind excess photoelectrons in the conduction band. These excess free electrons enhance the $\mathrm{ZnO}$ conductivity [37]. After switching off the UV, ambient species are chemisorbed again on the surface, and the persistent conductivity decays.

\subsection{Graphene and graphene oxide}

Graphene (GR), one of the most famous 2D materials, has attracted great interest over the last 15 years due to its remarkable electrical properties. In GR, a single layer of carbon atoms forms a 2D honeycomb-lattice structure with unique properties, such as high carrier mobility (up to $10^{6} \mathrm{~cm}^{2} \cdot \mathrm{V}^{-1} \cdot \mathrm{s}^{-1}$ ), low electrical surface resistivity $(0.1-6 \mathrm{k} \Omega / \square$ for a single layer with $97.7 \%$ optical transparency), and excellent chemical stability and mechanical strength [38]. Each carbon atom in graphene is bonded to neighboring $\mathrm{C}$ atoms by three strong $\sigma$ - 
bonds formed of hybridized $\mathrm{sp}^{2}$ orbitals, and an out-of-plane conjugated $\pi$ bond, which is responsible for GR's electrical conductivity.

The attainable characteristics of GR significantly depend on the layers' singularity. As the number of graphene layers is increased, its properties change dramatically into those of graphite. As a standard arbitrary threshold, backed up by the International Organization for Standards (ISO), more than ten graphene layers are considered graphite instead of graphene [39]. According to this standard, graphene as a single layer is abbreviated as $1 \mathrm{LG}$, bilayer graphene as 2LG, and few-layered graphene as FLG.

Graphene can be synthesized using the three main techniques of i) CVD growth on an appropriate substrate, ii) epitaxial growth on SiC surface, and iii) exfoliation of graphite flakes [38-40]. The bottom-up CVD and epitaxial growth techniques typically result in high quality and large wafer-scale areas of single and multiple-layer graphene films, suitable for high-performance carbon-based electronic applications. Although these methods are effective in terms of quality, their potential applications are limited by production costs.

In the other, inexpensive approach, a high volume of GR can be produced using a top-down, low-cost and facile technique based on exfoliation of bulk graphite into graphene nanoplates (GNPs). In the chemical exfoliation technique, chemicals are intercalated between the graphite layers, which weakens the cohesive van der Waals forces, causing graphite expansion and finally exfoliation [41, 42]. The final product of the exfoliation technique consists of a dispersion of synthesized graphene oxide (GO) flakes in a liquid, with a range of different thicknesses and lateral sizes that can further be dried in a powder form. Despite the simplicity and cost-effectiveness of this method, a significant portion of the final flakes have excess layers due to agglomeration between the particles. In this research, chemically exfoliated GNP has been used to develop the other nanocomposites.

Despite all the electrical advantages of GR, it also has disadvantageous characteristics that make it undesirable for direct use in some applications. Generally, graphene is highly hydrophobic with a strong tendency not to interact 
with other materials, making it almost non-dispersible in water or any organic solvent [43]. In addition, the GNPs undesirably agglomerate into large particles due to their high aspect-ratio and the existence of van der Waals forces between the flakes.

On the other hand, GO shows completely different characteristics compared to GR. Due to the presence of high-density oxygen functional groups (in the range of $40-70 \%$ oxygenated carbon atoms $[44,45]$ ) on its surface, GO is highly water dispersible, easy to interact with, and convenient to process. However, GO is an insulator and should be reduced to GR after the deposition process, usually by strong reductant chemicals like hydrazine, thermally [46], or by UV irradiation [47].

\section{$2.4 \mathrm{ZnO}$-graphene heterojunctions}

As explained in the previous chapter, epitaxial heterostructures are formed by strong covalent bonding between two dissimilar semiconductors, in which lattice matching is a critical parameter. Alternatively, van der Waals forces between a 2D material and other materials can also form stable heterostructures with interesting electronic properties. These van der Waals heterojunctions are formed by weaker van der Waals interactions between a 2D material and any passivated, dangling-bond-free material, independent of the lattice matching [48]. It is worth noting that the charge-transfer mechanism at the interface of these heterojunctions is by tunneling and hopping of electrons since the band structure is discontinuous at the interface [48].

In addition to van der Waals interactions, it has been shown that $\mathrm{ZnO}$ can also efficiently be grown on the GR surface $[5,8,9,49]$ with a relatively small lattice mismatch of about $2 \%[10,50]$. From an electrical point-of-view, this fairly well-matched interface between $\mathrm{ZnO}$ and graphene can lead to a Schottky or low-ohmic contact, depending on the doping level and heterojunction band alignment [7]. 



\section{Zinc oxide-graphene nanocomposites}

The low cost and simplicity of any novel material fabrication process greatly influence potential applications of the developed materials. Among different fabrication techniques, solution-based methods are simple, inexpensive and energy-efficient, with important practical industrializing capabilities. The main advantages of these techniques come from the low-temperature and atmospheric pressure growth conditions. This chapter describes how the chemical bath deposition technique was used to synthesize various substrate-free $\mathrm{ZnO} / \mathrm{GR}$ nanocomposites, mainly developed for electronics and photocatalysis applications. The synthesized nanocomposites, with random grown structures, have been investigated for improved dispersity, morphology, electrical and optical properties, and photocatalytic efficiency. Papers I and II address the material discussed in this chapter.

\subsection{Synthesis method}

Several synthesis methods of $\mathrm{ZnO}-\mathrm{NRs}$ have been realized during the last two decades. Most of these techniques require high temperature or sophisticated vacuum equipment for NRs growth that causes the final grown structures to more expensive, usually harder to industrialize, and applicable to limited areas. Chemical vapor deposition (CVD) is one of the most reported $\mathrm{ZnO}$-NRs growth techniques that can provide high-quality NRs at a high-temperature range of $500-950^{\circ} \mathrm{C}$, but at atmospheric pressure $[51,52]$. The high-temperature growth limits the usage of some potentially interesting substrates, especially flexible substrates, in such a growth process. Some other techniques, e.g., pulsed laser deposition (PLD) [53], vapor-liquid-solid (VLS) [52] and sputtering [54], operating at high vacuum conditions, result in high crystal quality but with a low 
deposition rate. Generally, using vacuum deposition techniques raises the production cost, and it is not favored in industry.

\subsubsection{Chemical bath deposition of $\mathrm{ZnO}$ nanorods}

Among the available growth techniques, solution-based growth methods, with advantages including simplicity, low-temperature and atmospheric pressure growth, are promising for synthesizing materials at a lower cost. Chemical bath deposition (CBD) and hydrothermal growth (HTG) are the two types of solutionbased growth techniques that sometimes are used conversely by mistake. While CBD refers to the growth process at low temperature and atmospheric pressure, HTG indicates the process at a higher temperature and higher pressures, using a metal or Teflon autoclave.

In CBD of ZnO-NRs, a saturated aqueous solution of the precursors is prepared. Then, an appropriately prepared substrate is suspended in the solution, and the temperature is elevated to a specific range. The CBD process includes two steps of nucleation and crystal growth. At the early stages, nuclei sites are formed by making a solid phase of $\mathrm{ZnO}$ from the growth solution. Subsequently, the $\mathrm{ZnO}$ crystal continues to grow epitaxially, forming the final crystal structure. However, a drawback of the CBD technique is that homogenous nucleation takes place in the bulk solution, causing many undesired grown structures and higher wastage of chemicals.

Several Zn precursors, including zinc nitrate, zinc acetate ${ }^{1}$ and zinc chloride $^{2}$, have been investigated for $\mathrm{CBD}$ of $\mathrm{ZnO}$, with a strong influence on the final grown morphologies [55-58]. In this thesis, we have always used an optimized bath solution of equimolar zinc nitrate ${ }^{3}$ and hexamethylenetetramine ${ }^{4}$ (HMT) for the $\mathrm{ZnO}-\mathrm{NRs}$ growth. The involved chemical reactions can be summarized as below:

\footnotetext{
${ }^{1} \mathrm{Zn}\left(\mathrm{CH}_{3} \mathrm{COO}\right)_{2} .2 \mathrm{H}_{2} \mathrm{O}$

${ }^{2} \mathrm{ZnCl}_{2}$

${ }^{3} \mathrm{Zn}\left(\mathrm{NO}_{3}\right)_{2} \cdot 6 \mathrm{H}_{2} \mathrm{O}$

${ }^{4} \mathrm{C}_{6} \mathrm{H}_{12} \mathrm{~N}_{4}$
} 


$$
\begin{aligned}
& \mathrm{Zn}\left(\mathrm{NO}_{3}\right)_{2} \rightarrow \mathrm{Zn}^{2+}+2 \mathrm{NO}_{3}^{-} \\
& \mathrm{C}_{6} \mathrm{H}_{12} \mathrm{~N}_{4}+6 \mathrm{H}_{2} \mathrm{O} \rightarrow 6 \mathrm{COH}_{2}+4 \mathrm{NH}_{3} \\
& \mathrm{NH}_{3}+\mathrm{H}_{2} \mathrm{O} \rightleftharpoons \mathrm{NH}_{4}^{+}+\mathrm{OH}^{-} \\
& \mathrm{Zn}^{2+}+2 \mathrm{OH}^{-} \rightarrow \mathrm{Zn}(\mathrm{OH})_{2} \\
& \mathrm{Zn}(\mathrm{OH})_{2} \rightarrow \mathrm{ZnO}+\mathrm{H}_{2} \mathrm{O}
\end{aligned}
$$

Dissolving of zinc nitrate and HMT provides $\mathrm{Zn}^{2+}$ and $\mathrm{OH}^{-}$ions in the aqueous solution (equations (3.1) to (3.3)), respectively. The intermediate species produced are sensitively dependent on the $\mathrm{pH}$ of the solution. Fig. 3-1 shows that the $\mathrm{Zn}^{2+}$ cations are dominant in the $\mathrm{pH}$ range of 6-7, while in a higher $\mathrm{pH}$ range of $10-11$, the $\mathrm{Zn}$ (II) hydroxide complex ions are more pronounced. During anisotropic c-axis growth of ZnO-NRs, the reactive cations diffuse in the bath solution and crystallize on the polar c-plane top face of ZnO-NRs.

In our experiment, two different concentrations of $25 \mathrm{mM}$ and $50 \mathrm{mM}$ zinc nitrate, with the equimolar HMT in DI water, were prepared as the CBD solution. In addition, the $\mathrm{pH}$ of the growth solutions was adjusted to the values of 6.6 and 11 by adding ammonia $\left(\mathrm{NH}_{3}\right)$. The growth temperature was set to $75{ }^{\circ} \mathrm{C}$ and maintained for $2 \mathrm{~h}$. In this approach, the seeded GRs (explained in the next part) were added to the growth solution under mild stirring.

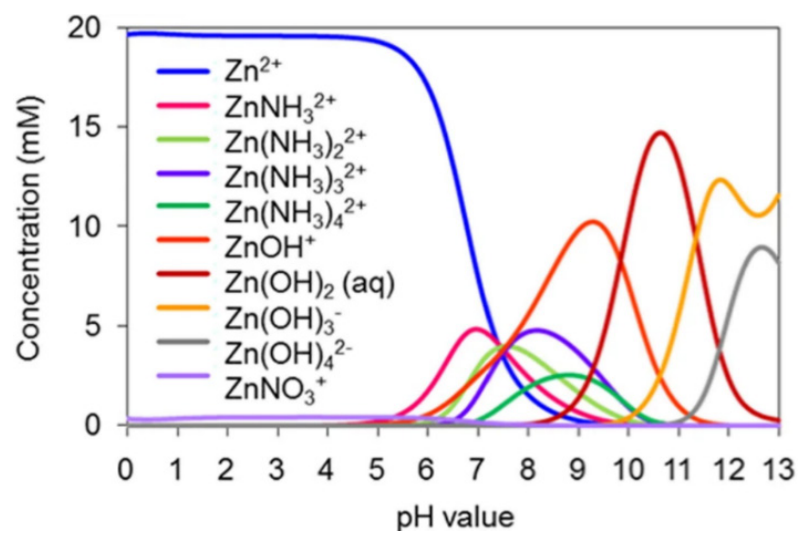

Fig. 3-1. Ionic species concentrations in different $\mathrm{pH}$ values for an aqueous solution of $20 \mathrm{mM}$ of zinc nitrate and HMT at $95{ }^{\circ} \mathrm{C}$, Reprinted from [59] under CC BY 4.0 License. 


\subsubsection{ZnO nanoparticles growth on GR nanoplates}

The $\mathrm{ZnO}$-NPs were prepared by hydroxylating the $\mathrm{Zn}^{2+}$ in an aqueous solution, using zinc acetate and $\mathrm{KOH}$. The reaction was performed in the presence of GR, to grow the NPs directly on the GR surface. First, an ultrasonicated dispersion of GR in DI water was mixed with a solution of zinc acetate while stirring. Subsequently, a dissolved $\mathrm{KOH}$ aqueous solution was added dropwise to the first solution at $60{ }^{\circ} \mathrm{C}$, while under ultrasonication. The seeding process continued in these conditions for $10 \mathrm{~min}$ to complete the growth. Then the decorated GR with $\mathrm{ZnO}-\mathrm{NPs}$ was washed with water and centrifuged at $3000 \mathrm{rpm}$ for $10 \mathrm{~min}$ several times and annealed at $300{ }^{\circ} \mathrm{C}$ for $30 \mathrm{~min}$.

The grown ZnO-NPs on the GR have two distinguished applications in our research. In the first application, the NPs played a seed layer role to assist the ZnO-NRs growth on the GR nanoplates in subsequent steps. Seeding the GR by NPs improves the GR hydrophobicity and will enhance the attachment between the GR and the ZnO-NRs. For this aim, a low NPs surface density was synthesized using a $500 \mathrm{mg} / \mathrm{L}$ dispersion of GR, a $5 \mathrm{mM}$ solution of zinc acetate and $25 \mathrm{mM}$ KOH. An SEM image in Fig. 3-2 shows typical seeded GRs with lowdensity NPs.

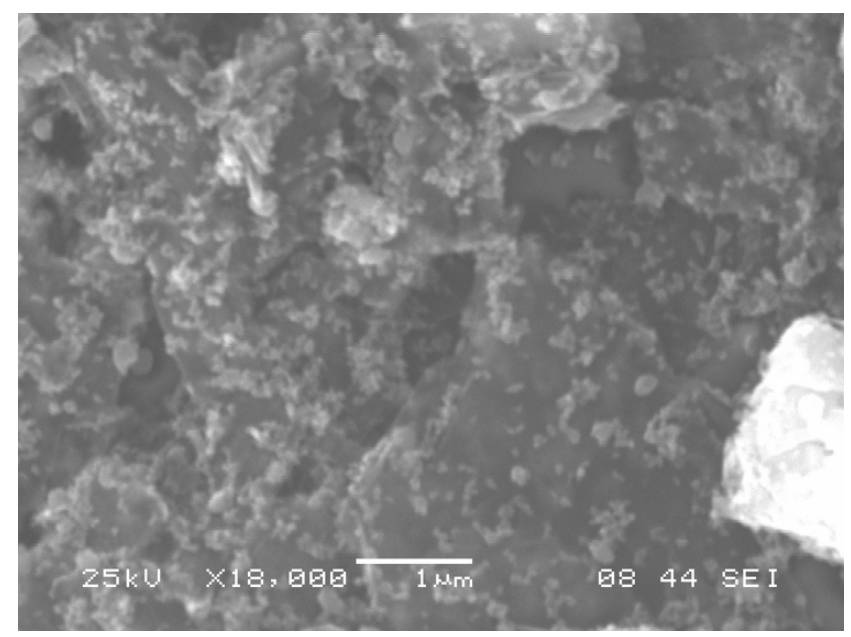

Fig. 3-2. SEM image of the GRs seeded with ZnO-NPs. Reprinted from Paper I [60]. 
In the other application, an optimized photocatalyst, with a high ZnO-to-GR weight ratio of 99:1, was fabricated. A $10 \mathrm{mg} / \mathrm{L}$ dispersion of GR powder, a 10 $\mathrm{mM}$ and $50 \mathrm{mM}$ aqueous solution of zinc acetate and $\mathrm{KOH}$, respectively, were used to synthesize the nanocomposite. The NPs here act as photogenerator of charge carriers by absorbing UV light and producing electron-hole pairs.

\subsubsection{Aluminum doping in $\mathrm{ZnO}$}

The Al-doped samples were synthesized by introducing a specific amount of aluminum nitrate ${ }^{5}$ into the growth solution. Dissolving the $\mathrm{Al}$ nitrate provides different Al(III) hydroxide complex species in the solution with a strong dependency on the solution's pH value. As shown in Fig. 3-3, the $\mathrm{Al}(\mathrm{OH})_{3}$ and anion $\mathrm{Al}(\mathrm{OH})_{4^{-}}$species are dominant at $\mathrm{pH}$ ranges of 6-7 and 10-11, respectively.

The amount of added $\mathrm{Al}$ nitrate was selected in a way that the final concentration of $2 \mathrm{mM}$ in the growth solution was met. It is essential to stir the solution for enough time before adding it to the growth solution. The aluminum nitrate solution is a weak acid, reducing the $\mathrm{pH}$ of the growth solution to a range of 55.5. This $\mathrm{pH}$ range is too low for the $\mathrm{ZnO}$ growth, and it can also dissolve the seed layer. Before adding the seeded GRs into the growth solution, the $\mathrm{pH}$ of the growth solution should be adjusted to desired values by adding enough ammonia.

The Al-doping of the ZnO-NPs was performed differently by immersing a pure piece of $\mathrm{Al}$ in the seed solution. The seed solution, explained in the previous part, has a high $\mathrm{pH}$ value of 13 that guarantees partial dissolution of the $\mathrm{Al}$ and the desired doping level of the ZnO-NPs [61].

${ }^{5} \mathrm{Al}\left(\mathrm{NO}_{3}\right)_{3} .9 \mathrm{H}_{2} \mathrm{O}$ 


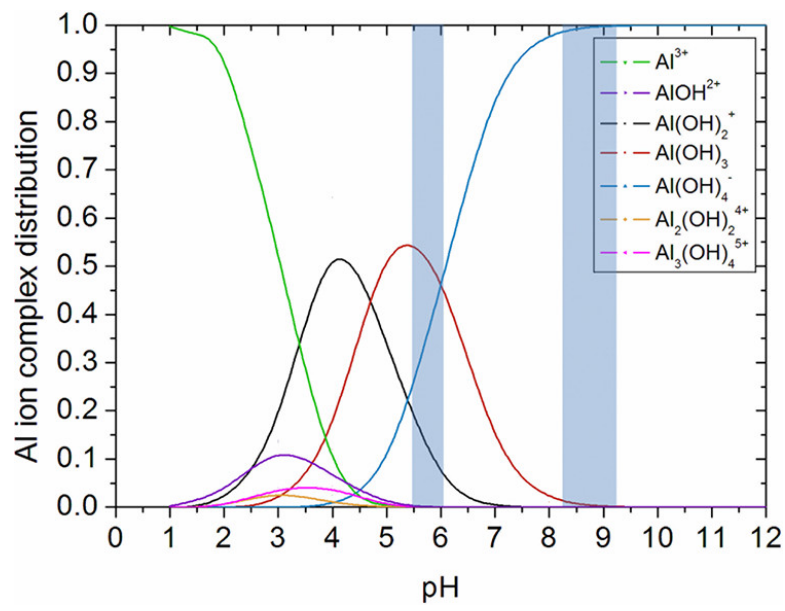

Fig. 3-3. Ionic species distribution in different $\mathrm{pH}$ values for an aqueous solution of 1.5 $\mathrm{mM}$ of aluminum nitrate at $90^{\circ} \mathrm{C}$. Reprinted with permission from [62]. Copyright 2017, American Chemical Society.

\subsubsection{Adding silver iodide into $\mathrm{ZnO} / \mathrm{GR}$ nanocomposites}

Silver iodide ${ }^{6}$ was introduced to the optimized photocatalytic ZnO-NPs/GR nanocomposite, with a high ZnO-to-GR ratio, to enhance the light absorption of the final product. First, the prepared $\mathrm{ZnO}$-NPs/GR nanocomposite was dispersed in DI water using an ultrasonic bath for $10 \mathrm{~min}$. Then, silver nitrate ${ }^{7}$ was added to the suspension, stirred for $30 \mathrm{~min}$. Subsequently, an aqueous sodium iodide ${ }^{8}$ solution was introduced dropwise into the solution and ultrasonicated for $1 \mathrm{~h}$. The weight ratio of $\mathrm{ZnO}-\mathrm{NPs} / \mathrm{GR}$ to $\mathrm{AgI}$ was chosen as three different values of $\mathrm{X}=10 \%, 20 \%$ and $30 \%$. The synthesized $\mathrm{ZnO} / \mathrm{GR} / \mathrm{Ag} / \mathrm{AgI}(\mathrm{X})$ nanocomposites were washed with DI water and acetone and collected by centrifugation, followed by drying in an oven at $75{ }^{\circ} \mathrm{C}$ for $6 \mathrm{~h}$.

\footnotetext{
${ }^{6} \mathrm{AgI}$

${ }^{7} \mathrm{AgNO}_{3}$

${ }^{8} \mathrm{NaI}$
} 


\subsection{Characterization methods}

The synthesized nanocomposites were analyzed using a large variety of experimental techniques. In this part, a brief description of the characterization techniques used in this thesis work is given. It can help the readers to understand better and interpret the results.

\subsubsection{Electron microscopy}

Field emission scanning electron microscopy (FE-SEM) and transmission electron microscopy (TEM) were used to study the structure and the morphology of the synthesized nanocomposites. In contrast to optical microscopes, electron microscopes use a focused electrons beam, with a relatively much shorter electron de Broglie wavelength than photons, to produce higher magnifications.

The Zeiss Sigma 500 Gemini microscope, equipped with a field emission gun operating at $10 \mathrm{kV}$ and Gemini Inlens secondary electron detector, was used to capture the SEM images. In SEM analysis, since the scattered electrons are detected on the same side as the incident beam, having enough conductivity in the samples for discharging is the only criterion to measure the specimens.

In TEM, electrons pass through the sample and the scattered beam is detected on the other side of the sample. Thus, the samples should be thin enough $(<100 \mathrm{~nm})$ to be electron transparent. This usually means more sample preparation steps are required for TEM measurement. The FEI Titan ${ }^{3}$ 60-300 microscope, equipped with image and probe Cs correctors and a monochromated high brightness XFEG gun, operated at $300 \mathrm{kV}$, was used for TEM imaging, scanning TEM (STEM) and high-angle annular dark-field imaging (HAADF).

Interaction between the incident electron beam in SEM and TEM and the subshell electrons in the sample atoms leads to X-ray emission, with a characteristic fingerprint of the specific elements in the sample. This method, called dispersive x-ray spectroscopy (EDS), was used to identify the elements' spatial distribution and perform chemical analysis of the sample. Using STEM-EDS, elemental map images with a resolution of a few nanometers can be obtained. 


\subsubsection{UV-Vis absorption spectroscopy}

In UV-Vis spectroscopy, the absorption of the medium at each wavelength is measured by transmitting monochromated light through a medium. The absorption spectra were measured using the spectrometer PerkinElmer Lambda 900 , equipped with two radiation sources of deuterium and tungsten lamps, and two PbS and PMT photodetectors. The spectrometer can measure in a wavelength range of 175 to $3300 \mathrm{~nm}$. The tungsten lamp provides radiation in the near-infrared (NIR) and visible (Vis) ranges, down to $320 \mathrm{~nm}$ wavelength, while the deuterium lamp covers the UV range. Dispersing the radiation beam at a grating produces a nearly monochromatic beam with which to irradiate the sample.

The beam alternatively passes in two different paths, one from the sample and one from a reference (usually an empty cuvette or the substrate), as shown schematically in Fig. 3-4. The transmitted intensities are detected as I and $\mathrm{I}_{0}$, respectively. The PMT and PbS photodetectors are used in the UV-Vis range up to $860 \mathrm{~nm}$ and in the NIR range, respectively. The transmittance (T) and the absorption (A) are calculated based on equations (2.1) and (3.7), only if the reflection from the samples is negligible; otherwise, the adsorption in (3.7) will be an approximation.

$$
\begin{aligned}
& T=\frac{I}{I_{0}} \\
& A=-\log T
\end{aligned}
$$

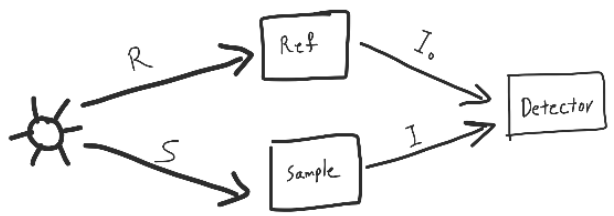

Fig. 3-4. Schematic of the monochromated beam paths, passing through the reference and the sample with corresponding transmittance intensities of $\mathrm{I}_{0}$ and $\mathrm{I}$. 


\subsubsection{Photoluminescence spectroscopy (PL)}

According to Planck's law of black-body radiation, hot materials can emit electromagnetic radiation, a phenomenon called incandescence. However, heating is not the only way to excite the materials. Alternatively, atoms and molecules can be excited by receiving energy in other forms, such as electrical current, impinging electrons, or absorbing photons. When these other forms of excitation result in spontaneous emission of light from a substance (cold-body emission), the names electroluminescence, cathodoluminescence, and photoluminescence are used, respectively.

In this work, a micro-PL ( $\mu$-PL) setup, schematically shown in Fig. 3-5, was used to study the optical characteristics of the samples. The PL measurement was conducted at room temperature with an $80 \mu \mathrm{W}, 266 \mathrm{~nm}$ excitation laser. A microscope lens focuses the laser beam on the sample with about $1 \mu \mathrm{m}$ spatial resolution. The photon energy of the excitation laser (here $3.4 \mathrm{eV}$ ) determines the excitation type in the experiment, band-to-band excitation (for the $\mathrm{ZnO}$ samples with the bandgap energy of $3.3 \mathrm{eV}$ ), or sub-bandgap excitation due to the defect levels. Alternatively, the intensity of the incident laser controls the density of photoexcited carriers. Radiative recombination between the photoexcited electron-hole pairs can occur through band-to-band recombination and sub-bandgap defect levels. This means the obtained PL spectrum gives direct information about the energy level structure and the system's impurity states [63].

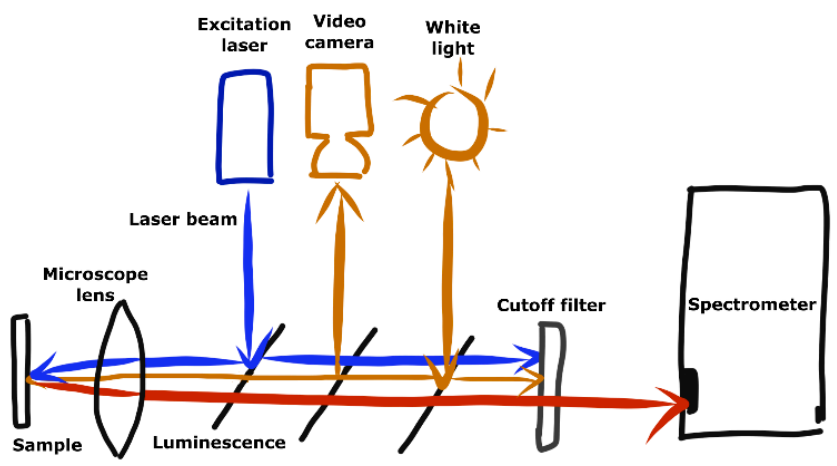

Fig. 3-5. Schematic of the $\mu$-PL setup. 


\subsubsection{Cathodoluminescence spectroscopy}

Very similar to PL, cathodoluminescence uses a focused electron beam to excite the sample locally. Then the luminescence is recorded as a spectrum. With a spatial resolution of tens of nanometers, this technique is relatively more local probing compared to $\mu$-PL. One difference between PL and cathodoluminescence is the lower penetration depth of electrons into the substance compared to photons. This leads to more surface-related data in cathodoluminescence compared to bulk-related PL measurement. The cathodoluminescence study was performed in a dedicated SEM at room temperature, operated at $5 \mathrm{kV}$. In this thesis, the acronym CL, often used for cathodoluminescence, is reserved for colloidal lithography, as described in the next chapter.

\subsubsection{X-ray diffraction analysis (XRD)}

$\mathrm{X}$-ray diffraction is a form of scattering from a periodic array with long-range order that ensures constructive interference at specific angles and so forms diffraction patterns. The diffraction pattern contains information about the atomic arrangement, the crystal plane orientation, and the element's fingerprint. The constructive interference angles from the parallel planes of a crystalline structure are calculated according to Bragg's law.

In this work, we used the PANalytical X'Pert Pro diffractometer, equipped with the Empyrean $\mathrm{Cu}$ X-ray tube, operating at $45 \mathrm{kV}$ and an electron current of $40 \mathrm{~mA}$, with the X-ray emission at the $\mathrm{Cu}$ Ka line with $1.5418740 \AA$ wavelength. An X'Celerator detector, in scanning line operation mode, and a nickel $\beta$-filter were used on the diffracted beam side.

\subsubsection{X-ray photoelectron spectroscopy (XPS)}

XPS is a photoelectric experiment in which a sample is irradiated with monochromatic X-ray, with a photon energy higher than the work function $\left(\varphi_{0}\right)$ of the sample, causing emission of electrons (so-called photoelectrons) from the sample. The kinetic energy ( $\mathrm{E}_{\mathrm{K}}$ ) of the emitted electrons is measured by a spectrometer in ultra-high vacuum (UHV) conditions. Knowing Ек, the binding energy 
$\left(\mathrm{E}_{\mathrm{B}}\right)$ of the electrons in the material can be calculated according to Einstein's equation (3.8).

$$
E_{K}=h v-E_{B}
$$

$E_{B}$ is defined as the energy difference between the total ground state energy of an atom and the total energy of a cation with a core hole. In contrast to the valence electrons, the core-level electrons are tightly bound to the nucleus in an atom and do not participate in chemical reactions. In XPS, only the core-level electrons, with an Ев smaller than the energy of the X-ray source, are probed. This detectable Ев range is $0-1200 \mathrm{eV}$ for a typical $\mathrm{Al} \mathrm{Ka} \mathrm{X-ray} \mathrm{source.} \mathrm{In} \mathrm{the}$ XPS spectra, each element in the sample shows a unique set of binding energies as a fingerprint, denoted by its relevant quantum energy level (n), the angular momentum quantum number (l), and the total angular momentum number $\left(\mathrm{j}=\mathrm{l}_{ \pm \mathrm{s}}\right.$ ) ( $\mathrm{s}$ is the spin quantum number).

Because of the short inelastic mean free path of electrons in materials, XPS analysis is extremely surface sensitive, with the highest conveyed information from a depth of a few atomic layers and a small fraction from deeper layers. The technique has a relatively low lateral resolution, about $150 \mathrm{~nm}$ to $15 \mu \mathrm{m}$, but an excellent detection limit of about 0.1 at\% for essentially all elements except hydrogen.

One important application of XPS is to analyze chemical bonding in complex samples, e.g., nanocomposites, by precisely determining the chemical shifts in the $\mathrm{E}_{\mathrm{B}}$ of the core electrons. Although the core-level electrons are not involved in chemical reactions, the electronegativity of the neighboring atoms can shift their binding energy. In general, a lower charge density around an atom shifts the $\mathrm{E}_{\mathrm{B}}$ of the core electrons toward higher energies.

\subsubsection{Surface resistivity measurements}

The surface resistivity was measured by the four-point probe method using a Keithley 4200-SCS semiconductor characterization system. The method is based on an in-line four-point array of probes, touching the sample surface with an equal distance (s) from each other. A current (I) passes from the outer probes 
while the potential difference between the inner probes $(\mathrm{V})$ is measured. For an infinite sample with infinitesimal thickness, the sheet resistivity $\left(\rho_{\mathrm{s}}\right)$ is calculated based on (3.9). For finite samples with finite thickness, depending on the ratio of the sample dimensions to the probe spacing $(\mathrm{d} / \mathrm{s})$ and the ratio of the sample thickness to the probe spacing (w/s), different correction factors (C) are required. A table of the corresponding correction factors can be found in [64].

$$
\rho_{s}=\frac{V}{I} \frac{\pi}{\ln 2}=\frac{V}{I} C, \quad C \cong 4.5324
$$

\subsubsection{Photoconductivity measurements}

The conductivity ( $\sigma$ ) of a substance is the product of the carrier density (n) and the carrier mobility $\left(\mu_{\mathrm{q}}\right)$ in the substance, according to (3.10), where $\mathrm{q}$ is the elementary charge. In semiconductors, increasing the carrier density by any form of excitation in the material, e.g., photoexcitation, leads to enhancing its conductivity (so-called photoconductivity).

$$
\sigma=n \cdot q \cdot \mu_{q}
$$

To measure the photoconductivity (PC) of the synthesized nanocomposites, the materials were sandwiched between two ITO electrodes with specified area and spacing to form an ITO/nanocomposite/ITO structure. A pulsed monochromatic excitation light, at room temperature and bias of $1 \mathrm{~V}$, irradiated the samples. A Keithley 2400 SourceMeter recorded the current during optical excitation with different wavelengths from 320 to $400 \mathrm{~nm}$.

Using the decay curve in the PC, after switching off the excitation light, the time-dependence of the PC of the samples can be calculated. The PC is proportional to the density of photoelectrons in the conduction band (CB), and it decays exponentially with time due to recombination of the photogenerated carriers [65]. The equations below relate the PC variation rate to the photoelectron lifetime, where $\sigma_{\mathrm{ph}}$ is the photoconductivity after subtracting the dark conductivity, $\tau_{d}$ is the decay time constant (photoelectron lifetime) and $\sigma_{0}$ is the observed PC directly after switching off the light. 


$$
\begin{aligned}
& \frac{d \sigma_{p h}}{d t}=-\frac{\sigma_{p h}}{\tau_{d}} \\
& \operatorname{Ln} \sigma_{p h}=-\frac{t}{\tau_{d}}+\operatorname{Ln} \sigma_{0}
\end{aligned}
$$

\subsubsection{Photodegradation efficiency measurements}

The photodegradation efficiency of the nanocomposites, which were developed for photocatalysis applications, was investigated by the degradation of Congo red (CR) dye under simulated solar light. A small amount of the nanocomposite (50 mg) was mixed with $100 \mathrm{ml}$ of CR-dye solution with an initial concentration of $20 \mathrm{mg} / \mathrm{l}$. After reaching the adsorption-desorption equilibrium conditions between the nanocomposite and the dye molecules in the dark, the mixture was exposed to the simulated solar light for $60 \mathrm{~min}$ in $15 \mathrm{~min}$ interval steps. Timeresolved UV-Vis absorption spectra of the remaining CR-dye were recorded at each step. The CR-dye has a specific adsorption peak at $497 \mathrm{~nm}$, which decays with irradiation time, verifying the degradation of the CR-dye.

The photodegradation efficiency was calculated based on the Beer-Lambert law, stating that the absorption is proportional to the dye concentration [66], using equation (3.13), where $\mathrm{A}_{0}$ is the initial absorption, and $\mathrm{A}$ is the absorption after irradiation.

$$
\text { Degradation }(\%)=\frac{A_{0}-A}{A_{0}} \times 100
$$

In addition, the degradation rate constant was also calculated based on the Langmuir-Hinshelwood's pseudo-first-order kinetic model [67]. It was achieved by linear fitting to the logarithm scale plot of the relative concentration of CRdye vs. time, according to the equation below: $\mathrm{C}_{0}$ is the initial concentration of dye and $\mathrm{C}(\mathrm{t})$ is the concentration of the dye after irradiation time $\mathrm{t}$.

$$
\text { Degradation rate constant }=\frac{\operatorname{Ln}\left(\frac{C_{0}}{C(t)}\right)}{t}
$$




\section{3 $\mathrm{ZnO}-\mathrm{NRs} / \mathrm{GR}$ for electronics applications}

The ZnO-NRs/GR nanocomposite was developed and assessed for higher electrical conductivity. At the beginning of this research project, the ambition was to fabricate conductive nanocomposites based on $\mathrm{ZnO}$ and GR for 3D-printed electronics. The advantages of such a composite stem from its high macroporosity, thermal and mechanical stability and cost-effectiveness, suitable for bulk printing applications. Many electronic components can be produced by additive manufacturing in the case of existing highly conductive and inexpensive printable materials. Here, we report the electrical and optical properties of our developed ZnO-NRs/GR nanocomposite and the effect of morphology and Al-doping on the properties of the final nanocomposites. This section is addressed in Paper I.

\subsubsection{Effects of pH on the morphology of $\mathrm{ZnO}-\mathrm{NRs} / \mathrm{GR}$}

The $\mathrm{ZnO}-\mathrm{NRs} / \mathrm{GR}$ nanocomposites were grown at two different $\mathrm{pH}$ values of 6.6 and 11, with and without Al-doping. The $\mathrm{pH}$ values were chosen to avoid unstable and opaque precipitation during the growth (Section 3.1.1). The results in Fig. 3-6 show that the growth of ZnO-NRs at pH 6.6 leads to a hexagonal, thicker (mean diameter $313 \mathrm{~nm}$ ), longer (1-2 $\mu \mathrm{m}$ ), and sparser structure compared to growth at $\mathrm{pH} 11$. Alternatively, growing at $\mathrm{pH} 11$ results in more needle-like NRs with a mean diameter and length of $196 \mathrm{~nm}$ and 0.5-1.5 $\mu \mathrm{m}$, respectively. In addition, the average density of NRs on the GR surface was increased from $1.4 \mu \mathrm{m}^{-2}$ to $2.4 \mu \mathrm{m}^{-2}$ in the corresponding growths at $\mathrm{pH} 6.6$ and 11 . 

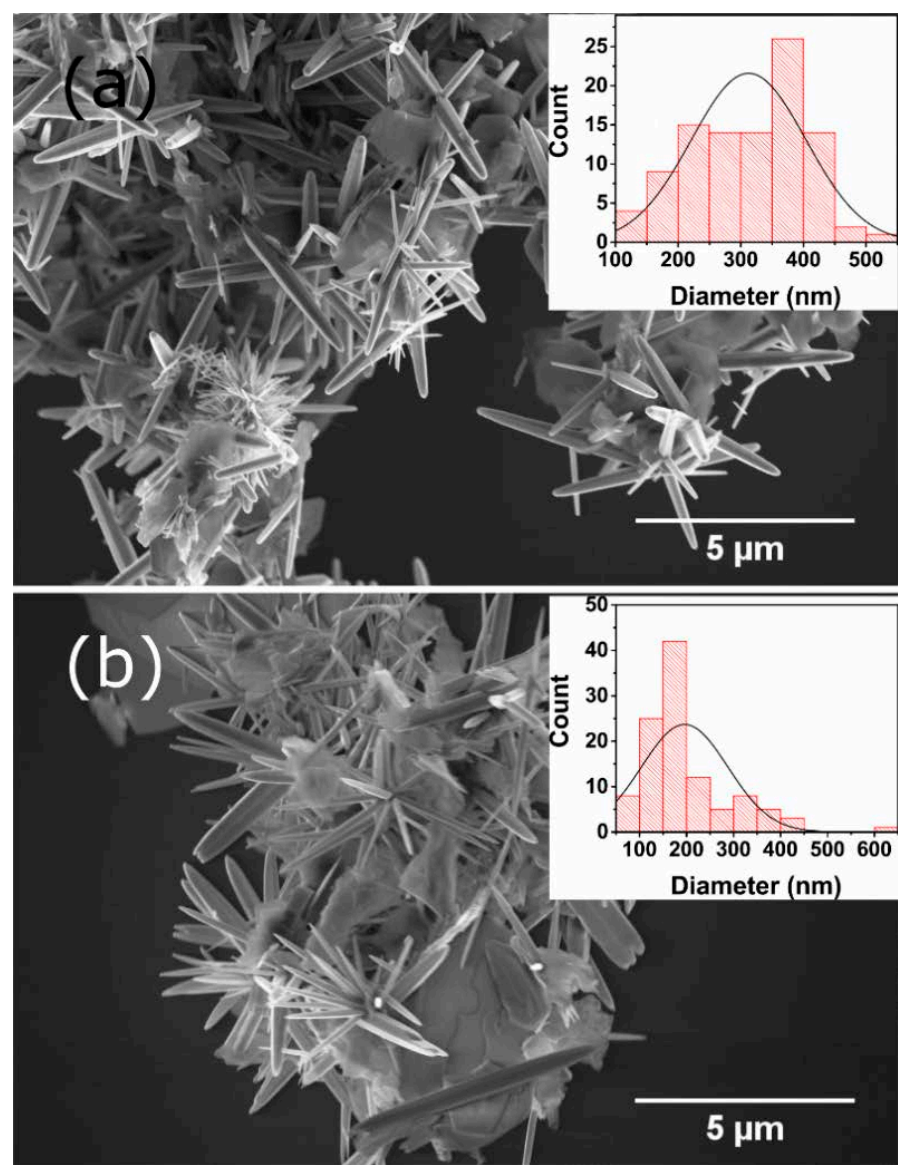

Fig. 3-6. SEM images of ZnO-NRs/GR nanocomposites grown at (a) pH 6.6 and (b) pH 11. The insets show the size distribution of the NR diameter. Reprinted from Paper I [60].

\subsubsection{Improving the conductivity of ZnO-NRs/GR by Al-dop- ing}

It is expected that the growth of wide bandgap $\mathrm{ZnO}-\mathrm{NRs}$ on GR will reduce the conductivity of the composite. To solve this issue, we degenerately doped the $\mathrm{ZnO}-\mathrm{NRs}$ by $0.5-1.5$ at\% $\mathrm{Al}$, as explained above in Section 3.1.3. Subsequently, the electrical resistivity of the samples was measured by the four-point probe technique on the deposited materials. A comparison of the measured resistivities is demonstrated in Fig. 3-7. It shows that spacing between the GRs, either 
by NPs or more pronounced by NRs, leads to higher resistivity. Also, it is readily observed that Al-doping strongly improved the nanocomposite conductivity. Since the GRs are the most conductive agent in the nanocomposite, it is reasonable that any introduced spacing between them will result in lower conductivity in the nanocomposite.

The very high resistivity in the undoped samples indicates a good dispersion of the GRs by ZnO-NRs, and also shows that the main portion of the current flows through the NRs. It is worth noting that even though the synthesized $\mathrm{ZnO}$ NRs/GR nanocomposites show relatively lower electrical conductivity, they occupy more space with relatively greater free space in their macroporous structure. This is very useful when mixing with other polymer materials, e.g., thermoplastics, for 3D-printing applications. In this perspective, we believe that the product of the conductivity and the macropores vol\% $\left(\sigma \times \frac{V_{\text {macropores }}}{V_{\text {nanocomposite }}} \times 100\right)$ is a better quantity to be compared between the nanocomposites.

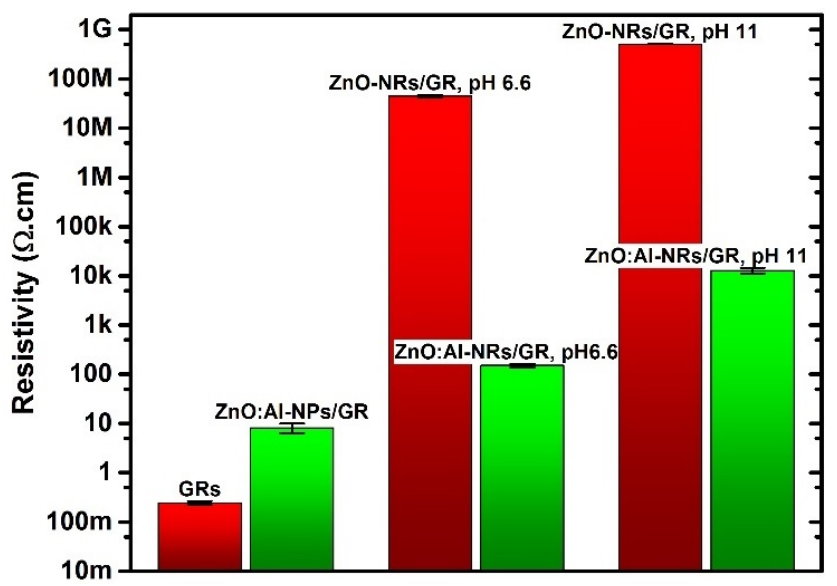

Fig. 3-7. Comparison of measured electrical resistivity of GRs, ZnO-NPs/GR and ZnONRs/GR nanocomposites grown at pH values of 6.6 and 11, with (green bars) and without (red bars) Al-doping. Reprinted from Paper I [60].

\subsubsection{Bonding quality between GR and $\mathrm{ZnO}-\mathrm{NRs}$}

The quality and the strength of bonding between the GR and the grown $\mathrm{ZnO}-$ NRs are essential aspects of our developed synthesis method, with a direct effect 
on the composite conductivity and its optical properties. The time-dependent UV-Vis spectroscopy reveals the existence of different material phases in the dispersion of the nanocomposite. The separate phases in the dispersion precipitate with different speeds, and consequently, can be identified using their different absorption spectra. Our results in Paper I show a uniform decrease in absorption for the ZnO-NRs/GR nanocomposites grown at higher $\mathrm{pH}$ values 11 and a non-uniform change for the samples grown at $\mathrm{pH}$ 6.6. This suggests that the growth at a higher $\mathrm{pH}$ solution leads to a better attachment of ZnO-NRs to the GRs.

\subsubsection{Optical properties of $\mathrm{ZnO} / \mathrm{GR}$ nanocomposites}

The effect of the $\mathrm{pH}$ growth solution and $\mathrm{Al}$-doping on the optical properties of the $\mathrm{ZnO} / \mathrm{GR}$ nanocomposites was studied by PL analysis. In Paper I, the PL data show variation in the near-bandedge emission (NBE) in the UV range, and deeplevel emission (DLE) in the visible spectrum for the various nanocomposites. Table 3-1 shows the ratio of NBE-to-DLE integrated intensities ( $\left.\mathrm{I}_{\mathrm{NBE}} / \mathrm{I}_{\mathrm{DLE}}\right)$ for different ZnO-NRs nanocomposites.

To summarize, growing at higher $\mathrm{pH} 11$ caused a significantly lower UV-tovisible emission ratio in all the samples. This lower emission ratio indicates higher defect levels concentration in the samples grown at $\mathrm{pH} 11$, due to the increased surface-to-volume ratio in the structure compared to growing at $\mathrm{pH}$ 6.6. It was also observed that Al-doping reduced the $\mathrm{I}_{\mathrm{NBE}} / \mathrm{I}_{\mathrm{DLE}}$ ratio for all samples. This reduction can be explained by the introduced $\mathrm{Al}$ donor levels in the bandgap and by the lower crystalline quality of the doped $\mathrm{ZnO}[68,69]$.

The results also show an increase in UV-to-visible emission ratio for $\mathrm{ZnO} / \mathrm{GR}$ composite samples compared to the pure $\mathrm{ZnO}$ structures. Reviewing the literature, this increase in the emission ratio can be attributed to the reduction of the surface defects concentration of the $\mathrm{ZnO}$ by the oxygen groups on the GR surface, or to resonant excitation of GR plasmons. More information can be found in Paper I. 
Table 3-1.The NBE-to-DLE integrated intensity ratio ( $\left.\mathrm{I}_{\mathrm{NBE}} / \mathrm{I}_{\mathrm{DLE}}\right)$ for different $\mathrm{ZnO}$ NRs/GR nanocomposites.

\begin{tabular}{lcc}
\hline & Grown at $\mathrm{pH}$ 6.6 & Grown at pH 11 \\
\hline ZnO-NRs & 2.46 & 0.15 \\
ZnO:Al-NRs & 1.78 & 0.11 \\
ZnO-NRs/GR & 4.25 & 0.46 \\
ZnO:Al-NRs/GR & 1.85 & 0.21 \\
\hline
\end{tabular}

In addition to PL analysis, the samples were also characterized with respect to the photogenerated carrier's lifetime and the spatial charge separation efficiency. Irradiating the samples with a photon energy close to the $\mathrm{ZnO}$ bandgap energy will generate free charge carriers in the $\mathrm{ZnO}$ conduction band (CB), causing increased electrical conductivity. By switching off the light, the number of free electrons in the $\mathrm{CB}$ will reduce due to recombination between the electronhole pairs, and it shows up in the sample conductivity decay. The more recombination in the sample, the faster the decay in the photoconductivity will be. Spatial separation of the photocarriers at the $\mathrm{ZnO}$-GR interface reduces the recombination rate. This reduced recombination rate leads to a longer photocarrier lifetime and, consequently, more persistent photoconductivity (PPC) in the samples.

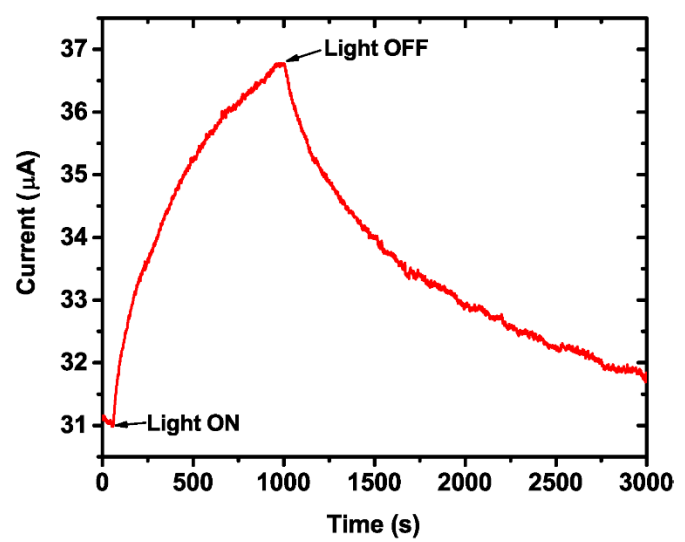

Fig. 3-8. A typical rise and fall curve of the current due to the PPC change in $\mathrm{ZnO} / \mathrm{GR}$ nanocomposites grown at $\mathrm{pH} 6.6$ under/after $350 \mathrm{~nm}$ illumination at $300 \mathrm{~K}$ and $1 \mathrm{~V}$ applied bias. Reprinted from my Lic. Thesis [70]. 
A typical rise and decay curve of the current due to the PPC change in $\mathrm{ZnO} / \mathrm{GR}$ nanocomposites is shown in Fig. 3-8. We did not detect any photoresponse in the samples, using excitation wavelengths longer than $400 \mathrm{~nm}$. Also, no PPC was observed in the highly Al-doped samples.

The time-dependence of the PPC of the undoped ZnO-NRs/GR samples with different morphologies, after $350 \mathrm{~nm}$ illumination at room temperature and under $1 \mathrm{~V}$ applied bias is shown in Fig. 3-9. Using Fig. 3-9 and the equation (3.12), the excess electron lifetime for the samples of ZnO-NRs/GR grown at $\mathrm{pH}$ 6.6 and $\mathrm{pH} \mathrm{11}$, and the pristine $\mathrm{ZnO}-\mathrm{NRs}$ were extracted as 877, 107 and $56 \mathrm{~s}$, respectively. The results show a more efficient charge transfer of photogenerated electrons from $\mathrm{ZnO}$ to the graphene in the sample grown at $\mathrm{pH} 6.6 \mathrm{com}-$ pared to the sample grown at $\mathrm{pH} 11$. This electron injection into the GR causes efficient spatial charge separation, a strongly reduced recombination rate, and consequently, an enhanced PPC.

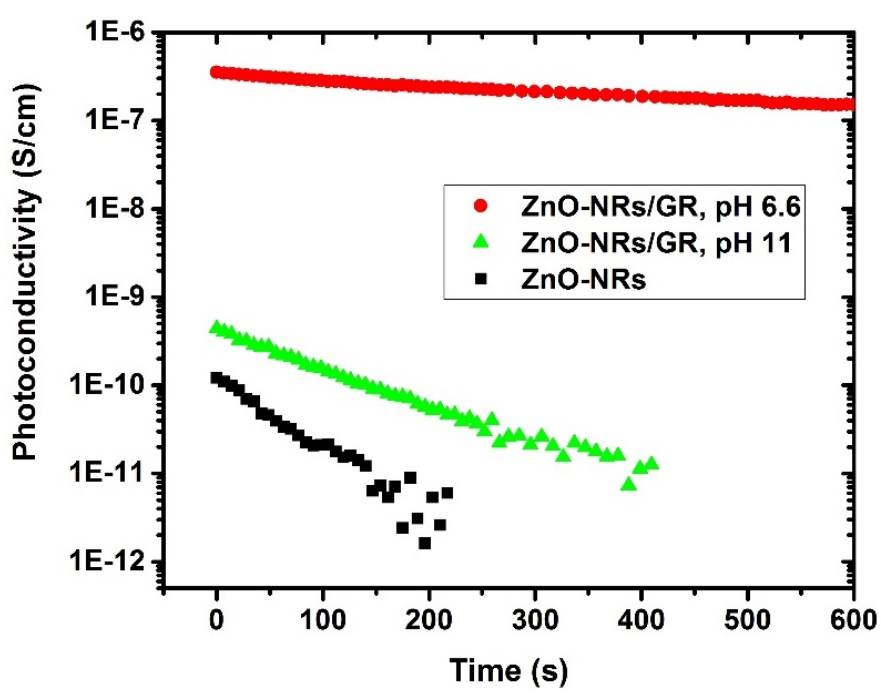

Fig. 3-9. Time-dependence of the PPC $\left(\sigma_{\mathrm{ph}}\right)$ after switching off the light for three different ZnO-NRs/GR nanocomposites, after $350 \mathrm{~nm}$ illumination at $300 \mathrm{~K}$ and $1 \mathrm{~V}$ applied bias. Reprinted from Paper I [60] under CC BY 3.0 License. 


\section{4 $\mathrm{ZnO}-\mathrm{NPs} /$ GR for photocatalytic applications}

Our last PPC results inspired us to use the developed $\mathrm{ZnO} / \mathrm{GR}$ nanocomposite for photocatalytic applications, an area where the charge separation phenomena and the photocarrier's lifetime are of great importance. In this application, the charge separation interfaces are more important than the bulk of the materials. For this reason, the nanoparticle form of $\mathrm{ZnO}$, instead of NRs, was chosen to increase the $\mathrm{ZnO}$-GR interfaces. In addition, the weight ratio of $\mathrm{ZnO}$ to $\mathrm{Gr}$ was increased to 99:1, as the primary light absorption material in the nanocomposite.

Although the tested $\mathrm{ZnO} /$ GR samples showed a promising charge separation capability, they are not active in the visible light range. The large bandgap of $\mathrm{ZnO}$ can only absorb incident wavelength in the UV range. This will limit the photocatalytic efficiency of these materials under solar irradiation, with its broad intensity peak in the visible range. To overcome this drawback, we introduced different weight ratios of lower bandgap (2.8 eV) AgI-NPs into the $\mathrm{ZnO} / \mathrm{GR}$ nanocomposites. The new $\mathrm{ZnO} / \mathrm{GR} / \mathrm{AgI}$ nanocomposites were analyzed with respect to their crystallinity, morphology, chemical structure, and photodegradation efficiency of an organic pollutant. This work is described in Paper II.

\subsubsection{Silver iodide}

Narrow bandgap silver-based semiconductors, including silver oxide, silver sulfide and silver halides, particularly AgI, with its excellent visible light absorption, have been widely studied for photocatalysis applications [71]. Historically, these Ag-based materials have been extensively used in photography for many years due to their highly sensitive light decomposing behavior. They will quickly decompose to metallic silver atoms $\left(\mathrm{Ag}^{0}\right)$ when exposed to light. However, this $\mathrm{Ag}^{+}$to $\mathrm{Ag}^{0}$ reduction will quickly be stopped at the initial stage of the reaction by a self-stabilizing mechanism. Clusters of reduced $\mathrm{Ag}^{0}$ atoms are formed on the AgI-NPs, preventing further decomposition of the semiconductor [71]. Therefore, we use the Ag/AgI in our notation instead of only AgI. 
The metallic $\mathrm{Ag}^{0}$ atoms play two roles in the AgI nanocomposites, i) trapping the other photogenerated electrons and stabilizing the AgI-NPs (as explained) and ii) acting as local surface plasmon resonators (LSPR) that lead to enhanced visible light absorption and higher photocatalysis performance. Our results show a shift in the absorption peak toward the visible range when adding AgI to the $\mathrm{ZnO} / \mathrm{GR}$ composite.

\subsubsection{Characterization of $\mathrm{ZnO} / \mathrm{GR} / \mathrm{Ag} / \mathrm{AgI}$ nanocomposites}

The XRD data and SEM images in Paper II, confirm the high crystalline quality and a uniform synthesis of the $\mathrm{ZnO} / \mathrm{GR} / \mathrm{Ag} / \mathrm{AgI}$ nanocomposites. The TEM image in Fig. 3-10 shows a conjunction of GR and ZnO-NPs and/or AgI-NPs in the nanocomposite. The GR nanoplates act as a substrate to assemble the NPs and also serve as an electrical bridge between them. The GRs can be considered as an electron sink for the photogenerated electrons in $\mathrm{ZnO}$ and $\mathrm{AgI}$, boosting the charge separation efficiency at the GR-NPs interface. This will positively affect the final photodegradation performance subsequently.

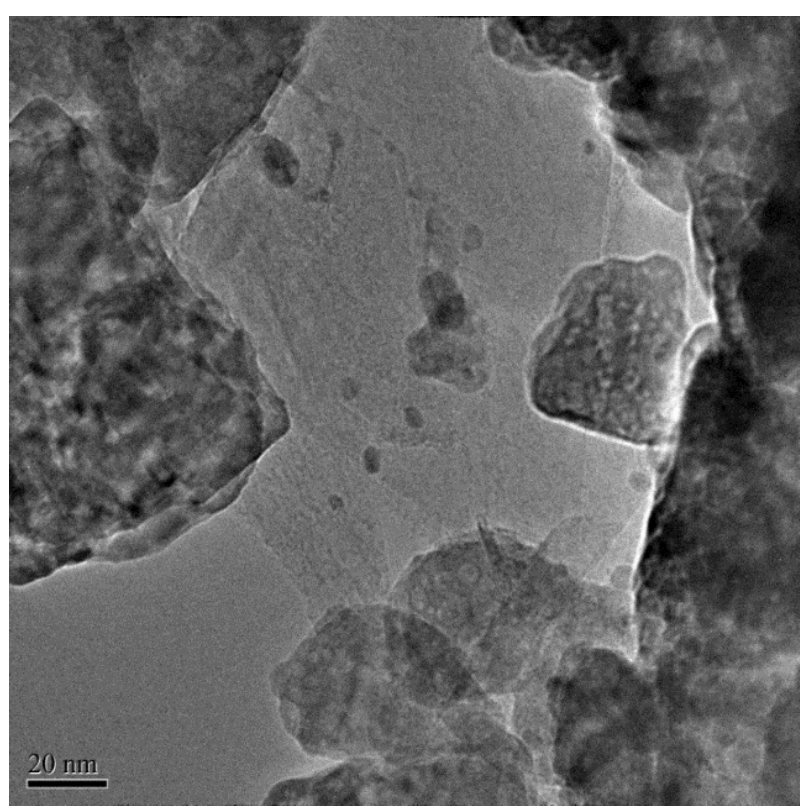

Fig. 3-10. TEM image of a $\mathrm{ZnO} / \mathrm{GR} / \mathrm{Ag} / \mathrm{AgI}$ (20\%) nanocomposite. 
The STEM-EDS analysis in Fig. 3-11 shows the elemental distribution of Zn, O, $\mathrm{Ag}$ and I atoms in the $\mathrm{ZnO} / \mathrm{GR} / \mathrm{Ag} / \mathrm{AgI}$ (20\%) nanocomposite. As we discussed earlier, the formation of the $\mathrm{Ag}^{0}$ clusters on the AgI-NPs is essential for highly efficient photocatalysts. Fig. 3-11(c) shows two displaced phases of Ag and I, indicating the formation of the $\mathrm{Ag}^{0}$ atoms on the AgI-NPs. This well elemental distribution in the nanocomposites can lead to higher photodegradation efficiency.

The existence of the metallic $\mathrm{Ag}^{0}$ atoms in the composition was further confirmed using XPS analysis. The binding energy of the $\mathrm{Ag}^{+}$shows a chemical shift toward lower energies compared to the $\mathrm{Ag}^{0}$. This negative $\mathrm{BE}$ shift in the $\mathrm{Ag} / \mathrm{Ag}^{+}$ systems is opposite to the typical BE shift of metal cations [72] that we explained in the previous section 3.2.6. The relevant XPS spectra can be found in Paper II.

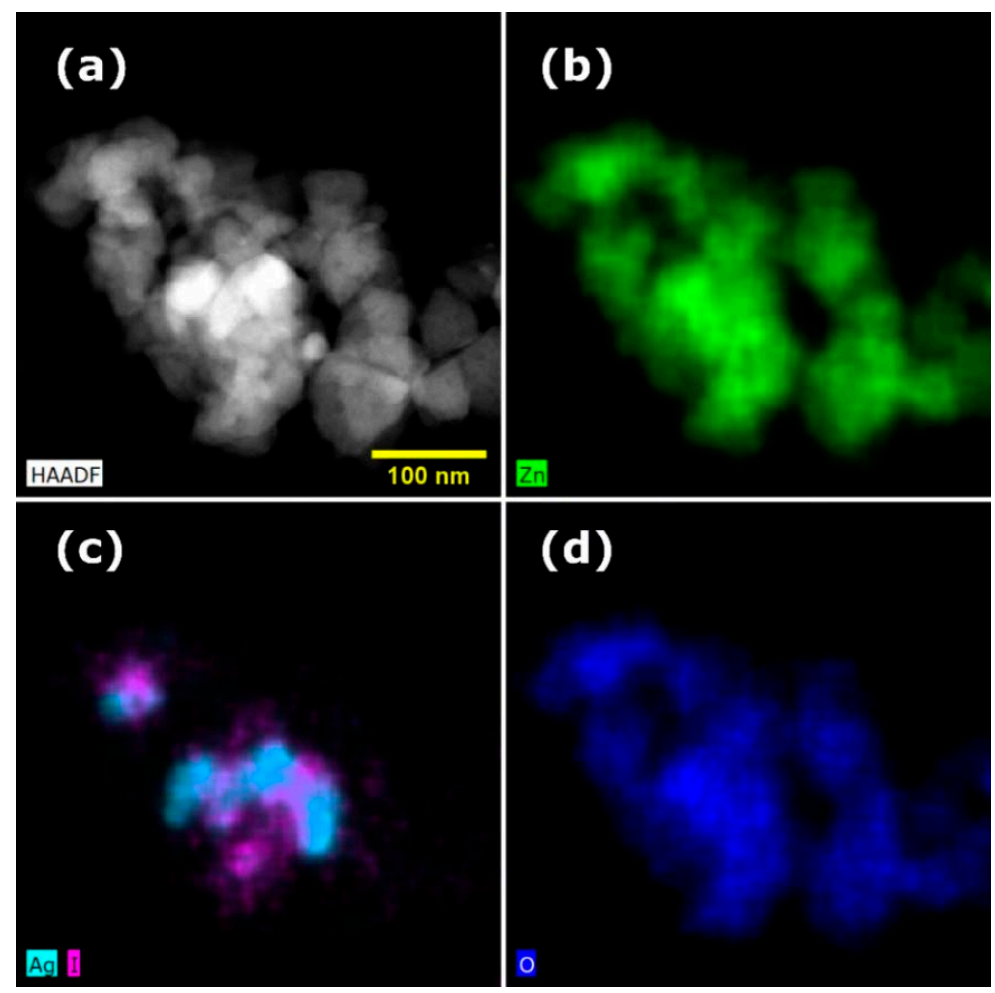

Fig. 3-11. (a) STEM-HAADF image of a ZnO/GR/Ag/AgI (20\%) nanocomposite. (b-d) Corresponding STEM-EDS elemental maps, displaying $\mathrm{Zn}, \mathrm{Ag} / \mathrm{I}$ and $\mathrm{O}$ distributions, respectively. Reprinted from my Lic. Thesis [70]. 


\subsubsection{Photocatalytic performance of the nanocomposites}

First, the optical absorption and the optical emission of the $\mathrm{ZnO} / \mathrm{GR} / \mathrm{Ag} / \mathrm{AgI}$ nanocomposites, with different weight percentages of $\mathrm{Ag} / \mathrm{AgI}$, were inspected by UV-Vis spectroscopy and cathodoluminescence spectroscopy. An absorption peak at $430 \mathrm{~nm}$ wavelength, corresponding to $2.8 \mathrm{eV}$ bandgap energy of AgI, was only detected in the samples with AgI-NPs. The cathodoluminescence spectra also confirmed the $430 \mathrm{~nm}$ wavelength emission in the AgI involved nanocomposites, as well as a broad emission with a peak centered at $600 \mathrm{~nm}$, related to $\mathrm{ZnO}$ defect levels. We found that the samples with a $20 \%$ weight ratio of $\mathrm{AgI}$ in the nanocomposite exhibit the highest absorption and emission at $430 \mathrm{~nm}$, which in turn is expected to show the highest photocatalytic performance.

The results of photodegradation of CR-dye under simulated solar light (explained in section 3.2.9) also confirm the highest photocatalytic efficiency for the $\mathrm{ZnO} / \mathrm{GR} / \mathrm{Ag} / \mathrm{AgI}$ (20\%) nanocomposite. Fig. 3-12 compares the photodegradation efficiency at different irradiation times for different nanocomposites. As observed in this graph, the degradation of CR-dye is negligible without using an appropriate photocatalyst.

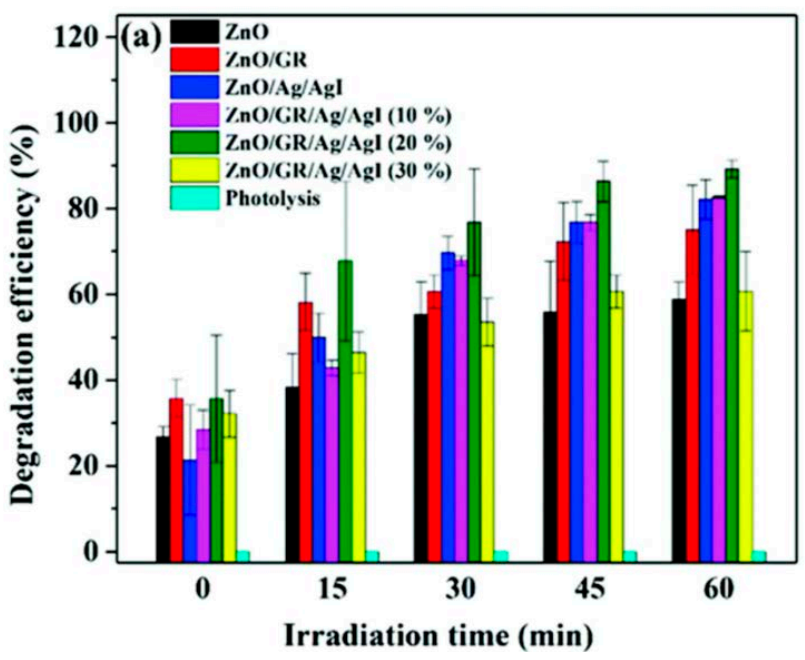

Fig. 3-12. Photodegradation efficiency of CR-dye at different irradiation times, using $\mathrm{ZnO}, \mathrm{ZnO} / \mathrm{GR}, \mathrm{ZnO} / \mathrm{Ag} / \mathrm{AgI}$, and $\mathrm{ZnO} / \mathrm{GR} / \mathrm{Ag} / \mathrm{AgI}$ photocatalysts with varying percentages of the weight of Ag/AgI. Reprinted from Paper II [73], published by The Royal Society of Chemistry. 
A proposed photodegradation mechanism, including the energy band structure, can be found in Paper II. Briefly described, both $\mathrm{ZnO}$ and AgI absorb light at different wavelengths and generate electrons and holes in their conduction band (CB) and valence band (VB). Because of the band offsets, the electrons in the CB of $\mathrm{AgI}$ are transferred to $\mathrm{CB}$ of $\mathrm{ZnO}$. The holes migrate in opposite direction, from the $\mathrm{VB}$ of $\mathrm{ZnO}$ to the $\mathrm{VB}$ of AgI. This charge separation enhances the carrier lifetime by reducing the recombination probability between the e/h pairs. Subsequently, the photogenerated electrons can migrate even further to the GR, causing strongly enhanced photocatalytic performance.

The separated electrons react with oxygen molecules to produce superoxide and hydroxyl radicals. The organic pollutant molecules (CR-dye) are decomposed by reacting to these active species in the solution. In the other reaction, the photogenerated holes can directly oxidize the adsorbed dye molecules and decompose the organic pollutants. 


\section{Template-assisted nanofabrication of $\mathrm{ZnO}-\mathrm{NRs} / \mathrm{GR}$}

In the previous chapter, we used CBD for the synthesis of the $\mathrm{ZnO} / \mathrm{GR}$ nanocomposites. Although various composites and nanostructures can be produced by this simple and inexpensive method, the final product usually consists of random structures, and it is hard to control their architecture and alignment. In many applications, especially for device fabrication, it is important to be able to control the nanostructure's alignment, ordering, density, and growth area.

This chapter describes a completely different approach, in which vertically aligned arrays of ZnO-NRs were grown on appropriately seeded $\mathrm{Si}$ wafer substrates. To accurately control the NRs surface density and selectively grow the NRs only in specific areas, colloidal lithography (CL) was employed to pattern the substrates in a relatively simple process.

To further improve the $\mathrm{ZnO}-\mathrm{NRs}$ alignment and fabricate electronic devices, a graphene oxide (GO) buffer layer was introduced between the Si substrate and the $\mathrm{ZnO}$ seed layer. This GO layer, after reduction to reduced graphene oxide (rGO), served as a compensator for the crystalline lattice mismatch between the $\mathrm{Si}$ and the $\mathrm{ZnO}$ and also as a back contact for device fabrication on insulating substrates.

\subsection{Synthesis methods}

The synthesis process of the aligned ZnO-NRs is essentially the same as described in the previous chapter. It includes seed layer preparation and ZnO-NRs growth on the seeded layers. However, a few changes in the chemicals used and the growth recipe were necessary to adapt the CBD technique for selective NR growth on different substrates. First, we needed to prepare a better GR dispersion. The GR nanoplates in the last part were purchased commercially in powder 
form and dispersed in DI water using ultrasonication. The prepared GR dispersion was not stable enough to make uniform spray-coated layers on the substrates required for this part of our research. So, we synthesized the GO using the improved Hummer's method, which will be explained below.

The ZnO-NP seed layer was also prepared differently by sol-gel and dipcoating techniques. The former solution of zinc acetate and $\mathrm{KOH}$ used to decorate the GR with ZnO-NPs, results in NP clusters and a non-uniform coating on the substrates. Therefore, we used the sol-gel solution to deposit perfectly uniform $\mathrm{ZnO}$ thin film layers on various substrates independently. Also, the CBD process was modified a little to achieve the highest alignment and quality of the ZnO-NRs.

\subsubsection{Synthesis of GO using the improved Hummer's method}

Graphene oxide (GO) was prepared using the improved Hummer's method according to the recipe described in [45]. The process is based on the chemical exfoliation of natural graphite flakes. Two types of natural and synthetic graphite are available commercially [74], and only the natural type can be used for GO synthesis.

First, $1 \mathrm{~g}$ of graphite flakes (mesh $-20+100$, with a particle size range of 150$850 \mu \mathrm{m}$ ) were oxidized using a mixture of concentrated sulfuric acid ${ }^{9}$ and phosphoric acid ${ }^{10}$ with a volume ratio of 9:1 $(60: 7 \mathrm{ml})$, stirring at $80{ }^{\circ} \mathrm{C}$ for $30 \mathrm{~min}$. The mixture cooled to room temperature, followed by $30 \mathrm{~min}$ ultrasonication (Fig. 4-1(a)). Subsequently, the mixture container was moved into an ice bath at $0{ }^{\circ} \mathrm{C}$, and $6 \mathrm{~g}$ of potassium permanganate ${ }^{11}$ was added to the mixture very slowly while stirring vigorously. The reaction is extremely exothermic; thus, caution is needed not to allow the mixture temperature to rise above $20{ }^{\circ} \mathrm{C}$ while adding the $\mathrm{KMnO}_{4}$. After this, the ice bath was removed, and the mixture was stirred at $35{ }^{\circ} \mathrm{C}$ for $2 \mathrm{~h}$. Subsequently, the mixture was diluted by adding $120 \mathrm{ml}$ of DI

\footnotetext{
${ }^{9} \mathrm{H}_{2} \mathrm{SO}_{4}(98 \%)$

${ }^{10} \mathrm{H}_{3} \mathrm{PO}_{4}(85 \%)$

${ }^{11} \mathrm{KMnO}_{4}$
} 
water into the acidic mixture. This diluting also produces much heat, increasing the mixture temperature to $95{ }^{\circ} \mathrm{C}$. The temperature was held at $95{ }^{\circ} \mathrm{C}$ for an extra $30 \mathrm{~min}$, using a heated bath while stirring. After cooling to room temperature, the mixture was diluted again with a solution of DI water and hydrogen peroxide $^{12}(240: 3 \mathrm{ml})$.

The final brownish solution contains the synthesized GO, non-exfoliated graphite flakes, residual sulfates, and oxidants at a very low pH (Fig. 4-1(b)). The solution was left overnight to precipitate the bigger particles, and the upper part of the solution was collected for further washing and purification. The collected mixture was centrifuged at $4500 \mathrm{rpm}$ for $30 \mathrm{~min}$ and washed with $\mathrm{HCl}$ solution in DI water (10\%) to remove any metals and other residuals. This washing process was repeated four times, followed by dispersing the collected precipitated solids in isopropanol alcohol. We noticed that the GO nanoplates were only collectible by centrifuging in an acidic solution ( $\mathrm{HCl}$ solution). As soon as the $\mathrm{pH}$ of the solution was neutralized by DI water washing, it was impossible to precipitate all the GOs, even at a higher centrifugal rotation speed of $12000 \mathrm{rpm}$.
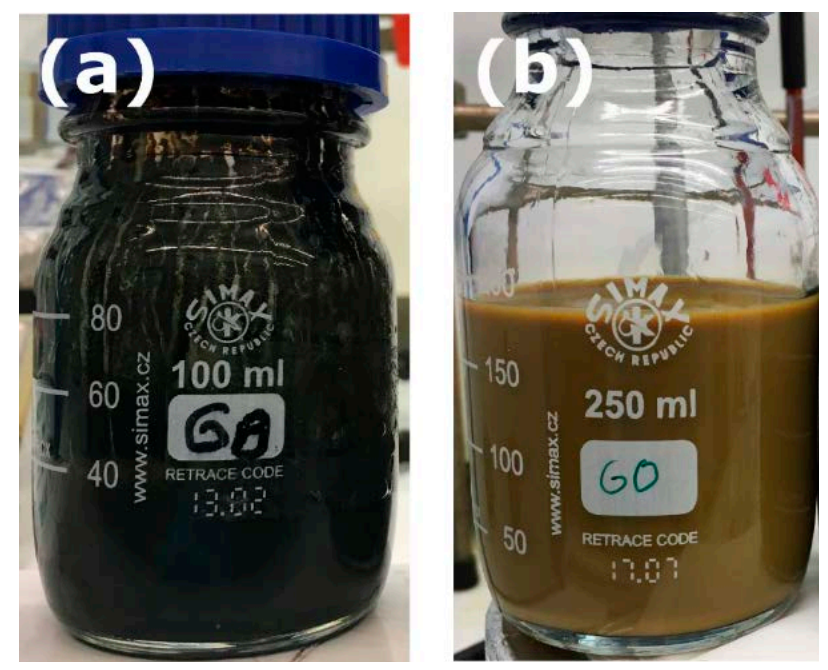

Fig. 4-1. (a) The mixture of graphite flakes in the acidic solution after stirring at $80{ }^{\circ} \mathrm{C}$ for 30 min. (b) The mixture of synthesized GO nanoplates in the acidic solution after terminating the reaction by adding $\mathrm{H}_{2} \mathrm{O}_{2}$.

${ }^{12} \mathrm{H}_{2} \mathrm{O}_{2}$ (30\%) 


\subsubsection{ZnO sol-gel solutions}

The ZnO-NPs synthesis procedure discussed in the previous chapter leads to NPs with size distribution in a range of 50-120 nm. The seed layers, produced by the spin coating of these NPs on a substrate, showed a non-uniform islandlike coating of NPs. Although changing the growth solvent to methanol and repeating the spin-coating step improved the seed layer surface coverage, it was not uniform enough for our nanofabrication process (Fig. 4-2(a)).

To improve the uniformity and the smoothness of the seed layers, a sol-gel solution of $8.25 \mathrm{~g}$ of zinc acetate and $2.26 \mathrm{ml}$ of monoethanolamine ${ }^{13}$ (ethanolamine), with an equimolar final concentration of $375 \mathrm{mM}$, in $100 \mathrm{ml}$ of pure ethanol was prepared $[75,76]$. The solution was stirred at $60{ }^{\circ} \mathrm{C}$ for $10 \mathrm{~h}$ and then at room temperature overnight. Fig. 4-2(b) shows an SEM image of a seed layer of ZnO-NPs, deposited on a Si substrate using the sol-gel method and dipcoating technique. Compared to the other method, the sol-gel process yields a highly uniform and dense seed layer with almost full surface coverage.

\subsubsection{CBD of ZnO-NRs on patterned substrates}

The same CBD method, explained in the previous chapter, was used for growing the $\mathrm{ZnO}-\mathrm{NRs}$, with and without $\mathrm{Al}$-doping. The only change was to increase the growth temperature from $75{ }^{\circ} \mathrm{C}$ to $95{ }^{\circ} \mathrm{C}$. Increasing the growth temperature causes a merging of the multiple closely packed narrow NRs into a single thicker NR. The growth of a single NR is crucial in our novel nanofabrication method, as will be explained below [77].

It is also worth noting that the $\mathrm{ZnO}-\mathrm{NRs}$ growth is performed on substrates in this approach, in contrast to the previous chapter. The prepared substrates were mounted upside down in the CBD solution to prevent any precipitant falling on the growth surface. We noticed that bubbles are formed in the solution during the experiment and may be trapped under the substrate, causing damaged or un-grown areas on the substrate surface. A little tilted mounting of the

\footnotetext{
${ }^{13} \mathrm{NH}_{2} \mathrm{CH}_{2} \mathrm{CH}_{2} \mathrm{OH}$
} 
substrate in the solution lets the bubbles gradually escape from under the substrate, improving growth uniformity.

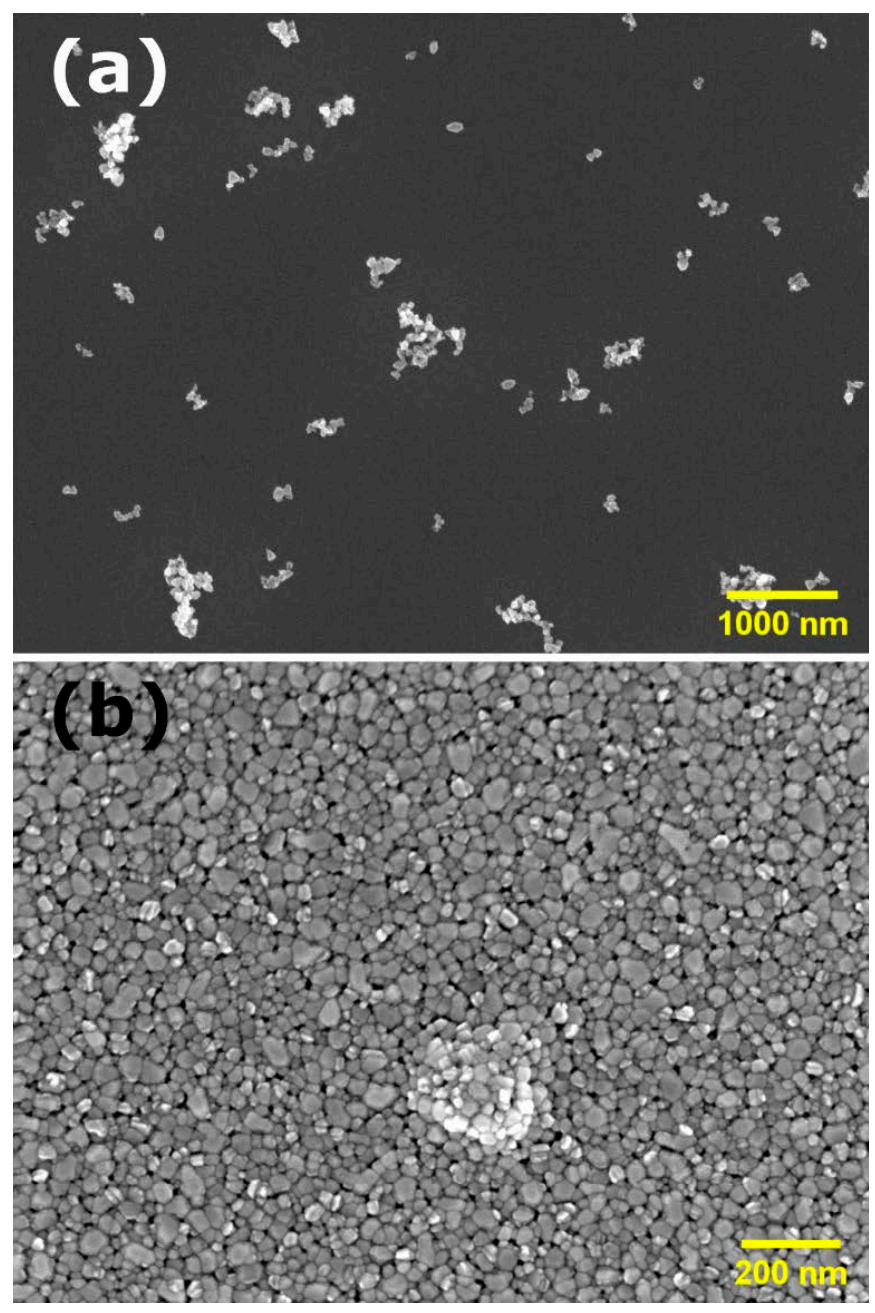

Fig. 4-2. SEM images of ZnO-NPs seed layers on Si substrate produced by (a) three times spin coating of the solution of zinc acetate and $\mathrm{KOH}$ according to the previous chapter, and (b) sol-gel and a dip-coating method.

\subsection{Nanofabrication of patterned substrates}

Our developed nanofabrication process consists of three steps: i) substrate preparation, ii) patterning the substate using colloidal lithography, and iii) CBD of 
ZnO-NRs on the patterned substrates. Two types of $\mathrm{Si} / \mathrm{ZnO}-\mathrm{NPs}$ and $\mathrm{Si} / \mathrm{GO} / \mathrm{ZnO}$-NPs seeded substrates were prepared using the sol-gel solution and a dip-coating technique. In the first structure, described in Paper III, the $\mathrm{ZnO}$ seed layer was directly formed on the Si substrate. In the second structure, discussed in Paper IV, a GO layer was deposited on the Si substrate prior to the $\mathrm{ZnO}$ seed layer. This GO layer serves as a buffer layer to compensate for the crystal lattice mismatch between the $\mathrm{Si}$ and the $\mathrm{ZnO}$, and also as a back contact for device fabrication on insulator substrates. The CL patterning process is basically the same for both substrate preparation methods.

\subsubsection{Spray coating of GO buffer layers}

A dispersion of the synthesized GO in N,N-Dimethylformamide ${ }^{14}$ (DMF) with a final concentration of $2 \mathrm{mg} / \mathrm{ml}$ was prepared by ultrasonication for $30 \mathrm{~min}$. Alternatively, isopropanol alcohol can also be used, although DMF results in more uniform layers. Both solutions remained stable for more than two weeks.

We used spray coating to deposit the GO dispersion on two-inch Si wafers. However, prior to that, the adhesion of GO to the Si substrate was enhanced by spin coating a thin layer of Adhesion Promoter AP3000 from the Advanced Electronics Materials, containing vinyltriacetoxysilane ${ }^{15}$ in 1-Methoxy-2-propanol solvent, at $4000 \mathrm{rpm}$ for $30 \mathrm{~s}$. It is critical to preheat the substrates at $120{ }^{\circ} \mathrm{C}$ for 5 min immediately before spin-coating to desorb surface moisture, and to perform soft baking at $120^{\circ} \mathrm{C}$ for $10 \mathrm{~min}$ after the coating.

Subsequently, the coated Si substrate was placed on a hotplate at $120{ }^{\circ} \mathrm{C}$ under a fume hood, and $20 \mathrm{ml}$ of the GO solution was pulse-wise sprayed on the sample with $1 \mathrm{~s}$ spraying and $3 \mathrm{~s}$ waiting times. An airbrush at a $10 \mathrm{~cm}$ distance from the sample, operating with nitrogen carrier gas at 2 bar pressure, was used. Fig. 4-3 shows the spray-coated GO on a Si wafer with and without substrate silanization. The silane-coated sample survived after five cycles of tape

\footnotetext{
${ }^{14} \mathrm{HCON}\left(\mathrm{CH}_{3}\right)_{2}$

${ }^{15}\left(\mathrm{CH}_{3} \mathrm{CO}_{2}\right)_{3} \mathrm{SiCH}=\mathrm{CH}_{2}$
} 
stripping, while the piece without adhesion promoter was delaminated during the liftoff process.

The thickness of the spray-coated GO layer was measured using a stylus Dektak-3ST profilometer to be in the range of 100-150 nm. Fig. $4-4$ shows a corresponding cross-sectional SEM image of a sample.

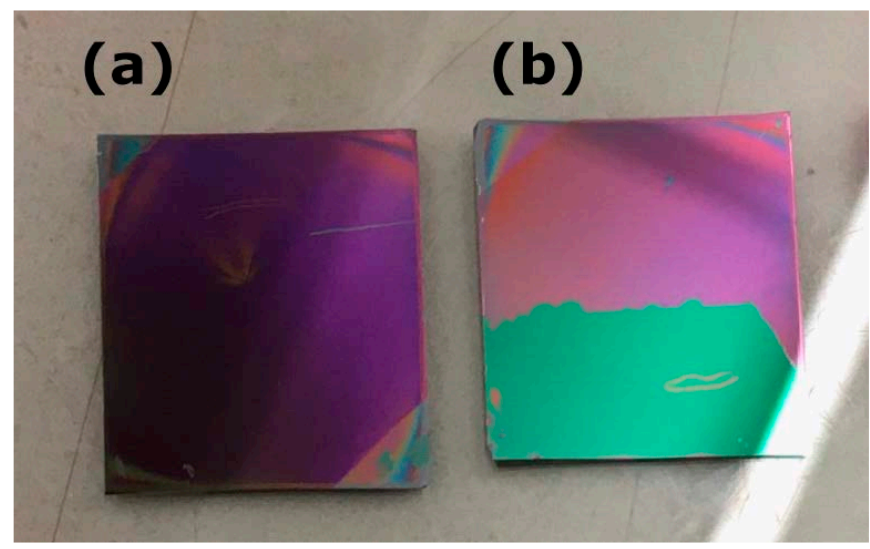

Fig. 4-3. Spray-coated GO on Si substrate (a) with, and (b) without silanization, after fivecycles of a tape stripping test. The samples also have a PMMA coating on top that will be explained below.

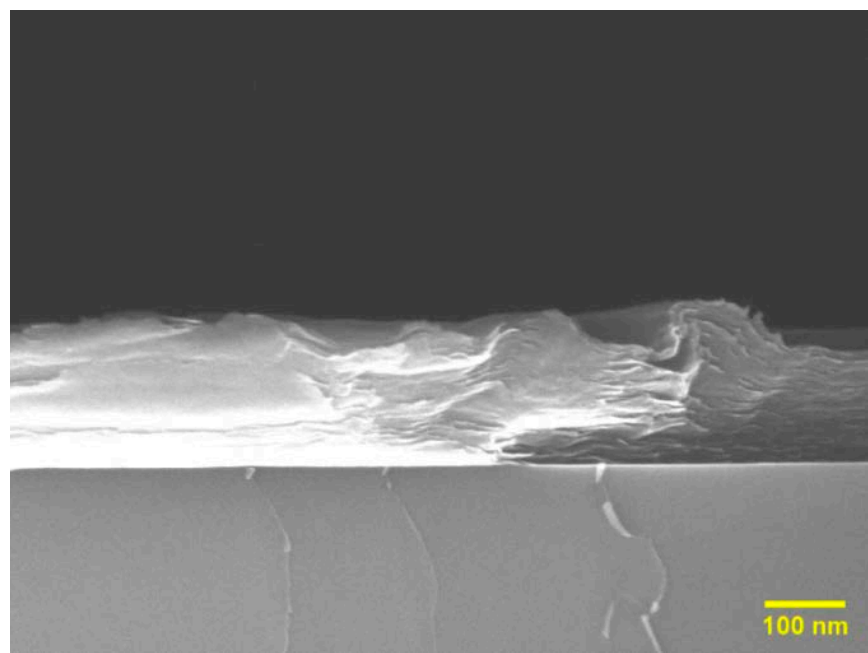

Fig. 4-4. Cross-sectional SEM image of spray-coated GO on Si substrate. 


\subsubsection{Dip-coating of the $\mathrm{ZnO}$ sol-gel solution}

A computer-controlled dip-coater was used to dip in and pull out the substrates into the ZnO-NPs sol-gel solution with a speed range of $10-30 \mathrm{~mm} / \mathrm{s}$ at room temperature. In this work, the dip-coating of the seed layers was carried out on two different substrates, a bare $\mathrm{Si}$ wafer and the GO coated $\mathrm{Si}$ (Si/GO). For the bare $\mathrm{Si}$ samples, the coating process was repeated twice, including an annealing step at $300{ }^{\circ} \mathrm{C}$ for $10 \mathrm{~min}$ after each dip-coating step.

However, the $\mathrm{Si} / \mathrm{GO}$ samples cannot tolerate high temperatures for a long time because of combustion of the GO layer in air. We measured a burning rate of $50-65 \mathrm{~nm} / \mathrm{min}$ for the GO layer at $300{ }^{\circ} \mathrm{C}$ in air. The annealing duration was limited only to $1 \mathrm{~min}$ to avoid oxidizing all the $\mathrm{C}$ atoms of the $\mathrm{GO}$ layer. In the end, a thickness of about 85-100 nm of the GO layer remained after the annealing process.

\subsubsection{Colloidal lithography (CL) patterning}

A polymer resist layer was spin coated on the seeded substrate to identify the selected growth areas on the samples. An array of nanoholes was then fabricated in the resist layer using the CL technique. CL allows for flexibility in the fabrication process and results in low-cost nanopatterning. Among other advantages, it is inexpensive, substrate independent, offers excellent control of the nanohole size, and is suitable for large wafer-scale area patterning [78-80].

In this work, we used a double-layer resist of a sacrificial PMMA ${ }^{16}$ electronbeam resist and then a positive MICROPOSIT S1805 photoresist on the seeded substrates. Various PMMA solutions in different solvents, including toluene, anisole, diethyl carbonate and DMF, were tested for spin coating of the first resist layer. Regarding the thickness and the uniformity of the layers, toluene results in a thick uniform layer, while anisole and diethyl carbonate solvents give thinner and uniform coating layers. In contrast, samples produced using DMF solvent showed a non-uniform coating.

${ }^{16}$ poly(methyl methacrylate), $\mathrm{MW}=950 \mathrm{k}$ 
After coating the resist layers, 5 min UV-Ozone treatment was necessary to improve the hydrophilicity of the resist for the following steps. A positively charged monolayer of $\mathrm{PDDA}^{17}$ was then pipetted on the sample surface and thoroughly rinsed with DI water after 1 min of settling. The positively charged monolayer of PDDA will electrostatically adhere the negatively charged polystyrene nanobeads (PS-NBs) to the substrate in the next step. The PS-NBs adhesion to the surface depends strongly on the number of the PDDA layers and will be reduced by multilayer-thick PDDA layers. Rinsing the PDDA layer under DI water flow for about $10 \mathrm{~s}$ improves the coated PS-NBs surface coverage.

A range of PS-NBs diameter sizes from $100 \mathrm{~nm}$ to $100 \mu \mathrm{m}$, suspended in water, is commercially available from microParticles $\mathrm{GmbH}$, Germany. In this thesis, we used the PS-NBs with mean diameters of 107, 140 and $320 \mathrm{~nm}$ for CL-patterning. Various concentrations of $0.1,0.02,0.01$ and 0.003 wt\% of the PS-NBs suspension in DI water were prepared by ultrasonication. Subsequently, a monolayer of PS-NBs was dispersed on the sample surface and gently rinsed with DI water after 1 min of settling. Fig. 4-5(a) shows a schematic of the sample at this stage.

\subsubsection{Thermal evaporation}

Thermal evaporation is a member of a bigger category of physical vapor deposition (PVD) techniques in which the materials are transported from a source to the growth substrate in the vapor phase. First, a material source is evaporated by receiving energy in various ways, including resistive heating in thermal evaporation, ion bombardment in sputtering, from an electron-beam in EBPVD, or a laser beam in pulsed laser deposition (PLD). In all these cases, a high or ultrahigh vacuum is needed to i) prevent the vaporized materials and the heated parts in the evaporator from oxidation, ii) reduce the materials' evaporation temperature, and iii) increase the mean free path of the vaporized particles, allowing transport without intermolecular collisions.

${ }^{17}$ poly(diallyldimethylammonium), MW $=(200-350) \mathrm{k}$ 
The vaporized particles are adsorbed on exposed surfaces inside the chamber, including the substrate, and condensation of material takes place. The thickness of the deposited materials is simultaneously monitored using a quartz crystal microbalance (QCM) and can be controlled by the deposition time.

In this thesis, a Balzers BA 510 evaporator was used to thermally evaporate a $30 \mathrm{~nm}$ thick $\mathrm{Al}$ layer on the samples at a high vacuum (HV) pressure of $6 \times 10^{-7}$ Torr (Fig. 4-5(b)). The evaporator was equipped with a diffusion pump with $2000 \mathrm{l}$ /s pumping speed and a combination of Pirani and cold cathode vacuum gauges to cover all the operating pressure ranges. The molten $\mathrm{Al}$ at high temperature is highly corrosive to the tungsten boat, penetrating it and alloying with the tungsten. To avoid this, the deposition rate was increased as high as possible to about 5-10 nm/s. After Al-coating, the Al-coated PS-NBs were removed by tape stripping using acrylic silicon-free Ultron 1009R tape, leaving behind open nanoholes in the Al film (Fig. 4-5(c)).

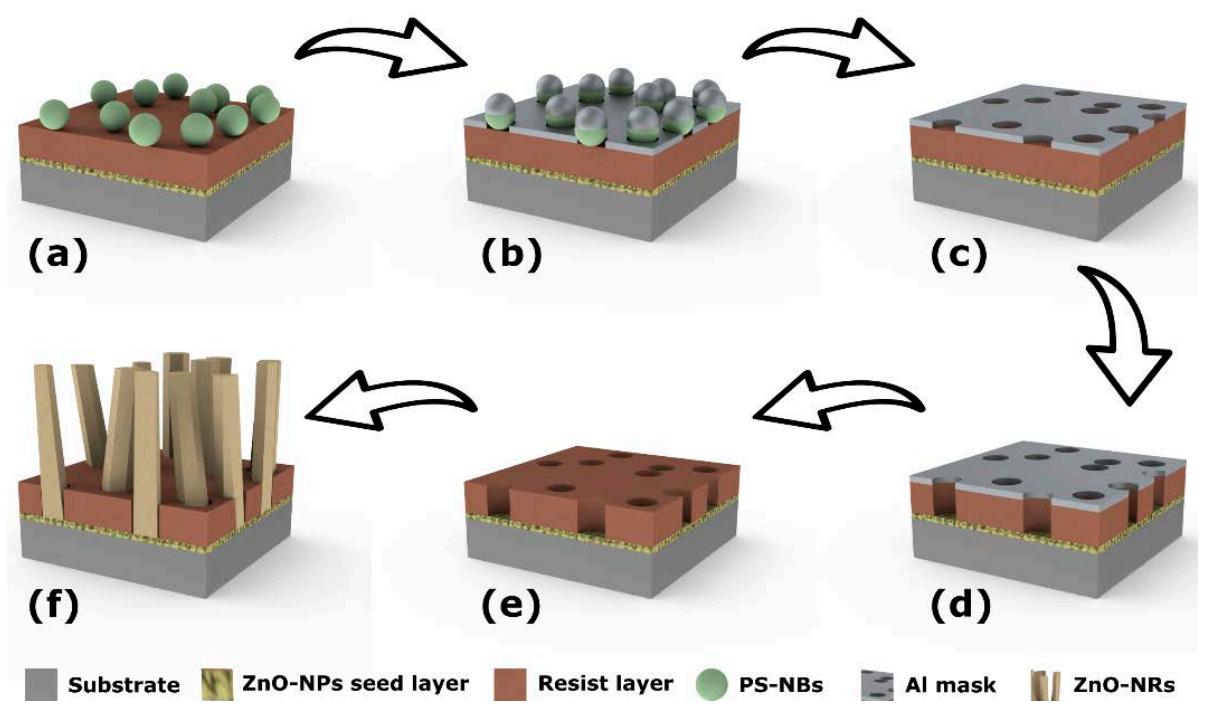

Fig. 4-5. Schematic of the CL nanofabrication process steps. (a) A substrate coated with a ZnO-NP seed layer, a double-layer polymer resist, and dispersed PS-NBs. (b) Thermal evaporation of an $\mathrm{Al}$ mask. (c) Removing the Al-coated PS-NBs by tape stripping. (d) Dry etching of the resist. (e) Wet etching of the Al mask. (f) Selective ZnO-NR growth in the holes of the patterned resist template. Reprinted from Paper III [81] under CC BY 4.0 License. 


\subsubsection{Dry etching}

Etching is a micro or nanofabrication technique that selectively removes materials from unprotected areas using chemical etchant species. In dry etching, ionized gases such as $\mathrm{SF}_{6}, \mathrm{Ar}, \mathrm{O}_{2}$ or $\mathrm{CF}_{4}$ are used as the etchant agents. The etchant gases are converted to plasma and bombard the surface in an applied electric field, causing the material to be removed from unprotected patterned areas. The reaction between the ions and the material can be purely physical (in sputter etching) or physical and chemical (in reactive ion etching) with a significantly higher etching rate.

In reactive ion etching (RIE), the plasma is formed by an applied radiofrequency (RF) electromagnetic field between two parallel plane electrodes, a grounded electrode on top and a negatively charged electrode at the bottom, under the sample. The positively charged ions are accelerated toward the sample under vacuum conditions, and because of the perpendicular electric field to the surface, etch away the materials directionally. Thus, the technique gives a highly anisotropic etching profile with a low lateral etching rate.

An RIE-Plasma Enhanced CVD from Advanced Vacuum, with operational parameters of pressure $150 \mathrm{mTorr}, \mathrm{O} 2$ flow $40 \mathrm{sccm}$, RF power $60 \mathrm{~W}$ and etching time $90 \mathrm{~s}$, was used to duplicate the $\mathrm{Al}$-mask pattern into the bottom resist layer. The patterned $\mathrm{Al}$ film works as an etching protection mask, defining the selected areas. Using these optimized operational parameters, etching rates of $3.3 \mathrm{~nm} / \mathrm{s}$ in the resist layer and $3.7 \mathrm{~nm} / \mathrm{s}$ in the GO layer were measured. Fig. 4-5(d) shows the etched nanoholes in the resist layer down to the $\mathrm{ZnO}$ seed layer.

\subsubsection{Wet etching}

In wet etching, a particular chemical solution etches a target material (usually a metal) without dissolving the others. Opposite to RIE, wet etching is usually isotropic with simultaneous lateral etching. In our research, the Al metal mask was removed at room temperature, using a particular etchant solution of $\mathrm{KOH}$ and 
potassium hexacyanoferrate III ${ }^{18}$ in DI water with respective concentrations of $30 \mathrm{mM}$ and $50 \mathrm{mM}$ for $1 \mathrm{~min}$. This chemical solution etches the Al layer without damaging the exposed $\mathrm{ZnO}$ seed layer [82]. After thoroughly rinsing with DI water and blow-drying with nitrogen, the nanopatterned samples are ready for the CBD of ZnO-NRs, as previously explained in section 4.1.3.

\subsection{Characterization methods - Atomic force microscopy}

In addition to the analytical techniques demonstrated in the previous chapter, atomic force microscopy (AFM) was extensively used to study the roughness and morphology of the seed layers. AFM is a part of a large family of scanning probe microscopy (SPM) techniques used for topography and local surface interactions studies and manipulating the surface structure [83]. The technique is based on probing the interactions (mechanical, electrical or chemical) between a sharp tip and a surface at an atomically-close distance. The system consists of a $\mathrm{Si}$ or $\mathrm{Si}_{3} \mathrm{~N}_{4}$ vibrational cantilever with a sharp tip that oscillates close to its resonance frequency $\left(\mathrm{f}_{0}\right)$ in tapping-mode operation. The $\mathrm{f}_{0}$ sensitively shifts due to the tip-surface interactions while approaching a surface. A photodetector measures a laser beam deflection from the cantilever, determining the frequency shift and measuring the tip Z-displacement. A feedback control loop mechanism and a piezoelectric Z-actuator maintain the tip-surface distance in a defined safe margin and record the displacements. Fig. 4-6 shows a schematic of an AFM system.

We used the Veeco Dimension 3100 AFM, equipped with a quad photoreactor, an acoustical-vibrational isolation table with hood, and a piezo scan head with $90 \mu \mathrm{m} x-y$ and $6 \mu \mathrm{m}$ movement range which can operate in contact and tapping modes. Fig. 4-7 demonstrates an AFM image of the synthesized GO deposited on a Si wafer surface and the corresponding liner profile scan.

${ }^{18} \mathrm{~K}_{3} \mathrm{Fe}(\mathrm{CN})_{6}$ 


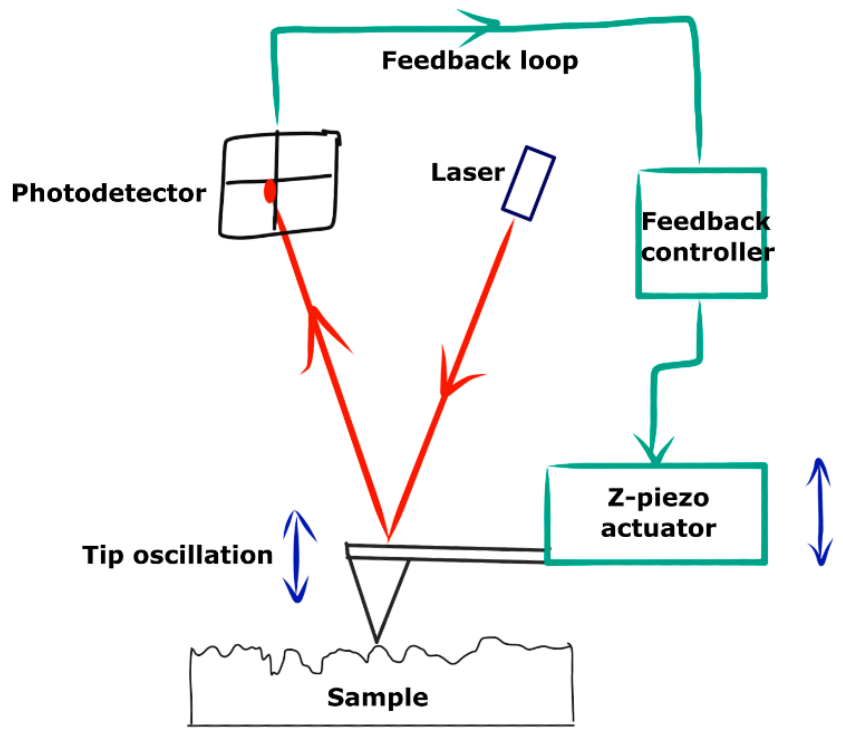

Fig. 4-6. AFM system schema.

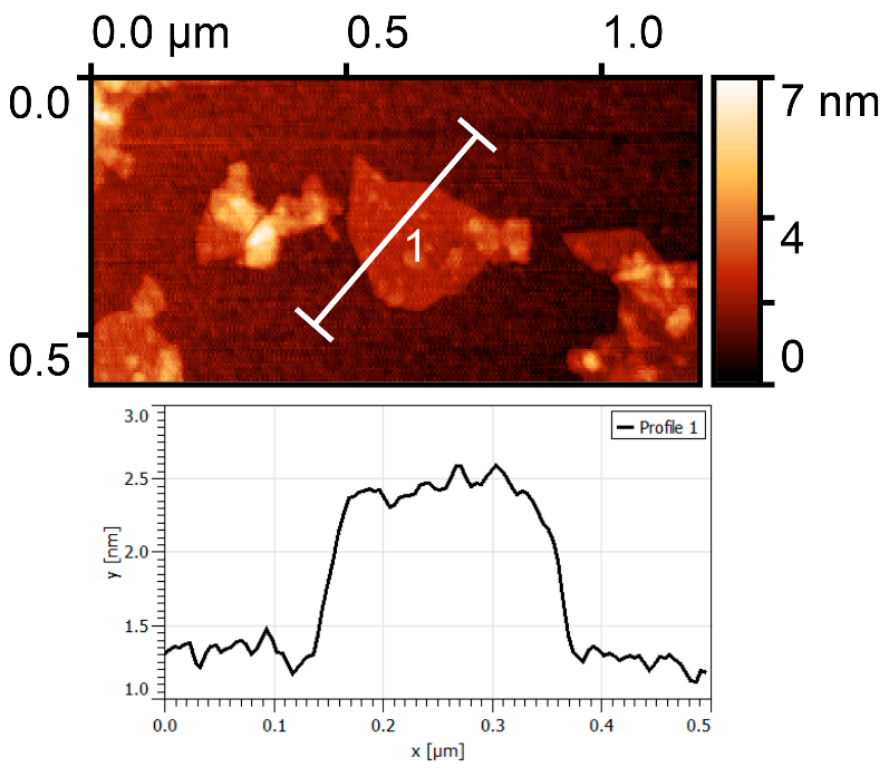

Fig. 4-7. AFM image of a GO nanoplate on Si wafer. The bottom graph shows a liner profile scan of the GO nanoplate with a thickness of about $1 \mathrm{~nm}$. 


\subsection{Characterization of the CL-patterned substrates}

A full detailed characterization of the CL-patterned substrates has been reported in Paper III and IV. Here, more detailed information is provided to fill the gap between these two works and connect them logically.

\subsubsection{Why using $\mathrm{Si} / \mathrm{GO}$ substrates?}

The CL method was tested on different substrates, including fluorine-doped tin oxide (FTO) and Ti and Au-coated glass substrates. An electrically conductive substrate is required to make a device. The other requirement is the stability of the substrate during the fabrication process. On the other hand, growing on $\mathrm{Si}$ has the significant advantage of being integrated with the well-developed Si industry.

Testing the CL on FTO substrates was not very successful due to the relatively high surface roughness of the FTO. High points on the surface apexes are usually exposed through the resist layer before etching, and depths are rarely accessible through the patterned nanoholes. A cross-section SEM image of a typical CL-patterned resist layer on an FTO substrate is shown in Fig. 4-8.

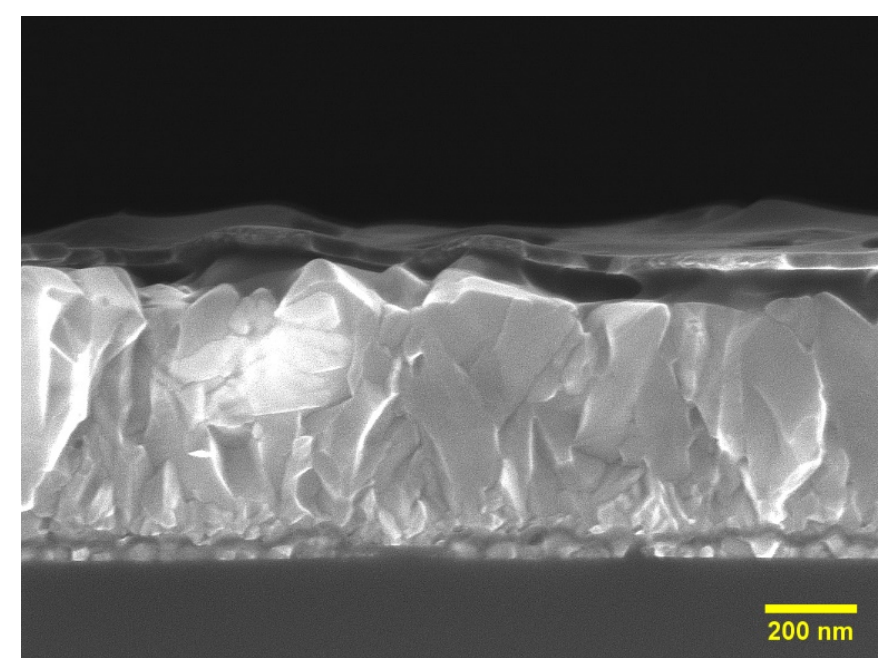

Fig. 4-8. Cross-sectional SEM image of the CL-patterned resist layer on FTO surface before wet-etching of the $\mathrm{Al}$ mask. 
The metal-coated substrates were not stable during the wet-etching process, and it caused delamination of the resist layer due to under-etching of the metal. Due to these practical limitations, the GO-coated Si was chosen as the basis for the CL patterning. However, bare GO is not a suitable nucleation surface for CBD of $\mathrm{ZnO}$, and it should be activated by the $\mathrm{ZnO}$-seed layer.

\subsubsection{Improved $\mathrm{ZnO}$ seed layers by dip-coating}

The $\mathrm{ZnO}$ seed layers with single and double dip-coating steps were produced and studied with respect to their smoothness, crystalline quality, and their influence on the grown $\mathrm{ZnO}$-NRs. For the $\mathrm{Si} / \mathrm{ZnO}$ samples, repeating the dip-coating process improved the smoothness and the crystal grain size of the seed layer. The AFM images in Fig. 4-6 show single-dip-coated seed layers with RMS roughness of $1.2 \mathrm{~nm}$ and small particle grain boundaries. In comparison, the double-dip-coated samples have a smoother surface with an RMS roughness of $0.9 \mathrm{~nm}$ and larger grain boundaries.
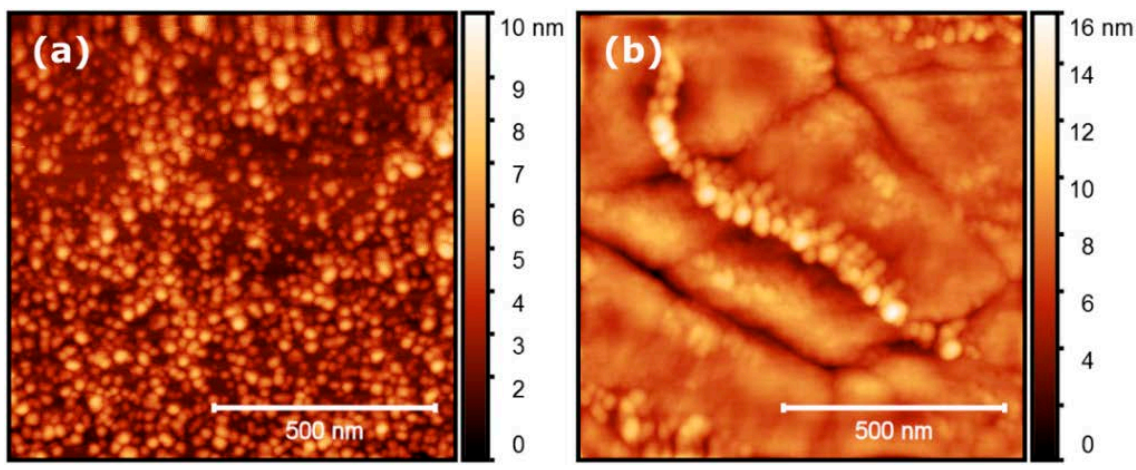

Fig. 4-6. AFM images of $\mathrm{ZnO}$ seed layers prepared on a Si wafer by a sol-gel method by (a) single and (b) double dip-coating. The samples were annealed at $300{ }^{\circ} \mathrm{C}$ after each coating step. A background has been subtracted from the AFM images.

From the literature, it is well understood that the crystal grain boundaries are increased by the thickness of the deposited layers [26, 84]. In our case, repeating the deposition process linearly increased the layers' thickness. In contrast, the presence of impurities during layer deposition and the annealing process reduces the grain sizes [85]. The size of the grain boundaries is important for our vertically aligned single NR growth through the patterned nanoholes. 
Although the smoothness and the grain size of the seed layers were improved by multiple dip-coating, the XRD analysis did not confirm any enhancement in the desired (002) crystalline orientation. This result suggests that the self-forming process of polycrystalline ZnO-NPs is strongly dependent on the substrate crystallinity and cannot be improved only by increasing the layer thickness.

To compensate the crystal lattice mismatch between the Si substrate and the $\mathrm{ZnO}$ seed layer, a GO buffer layer was introduced on the Si surface by spray coating, as explained in section 4.2.1. The AFM data show higher waviness in the GO layer due to the imperfectness of the spray-coating process with an RMS roughness of about $11 \mathrm{~nm}$. Fig. 4-9 shows the AFM image of a ZnO-NPs seed layer on a $\mathrm{Si} / \mathrm{GO}$ sample after annealing at $300^{\circ} \mathrm{C}$ for $1 \mathrm{~min}$ with an $\mathrm{RMS}$ roughness of $4 \mathrm{~nm}$.

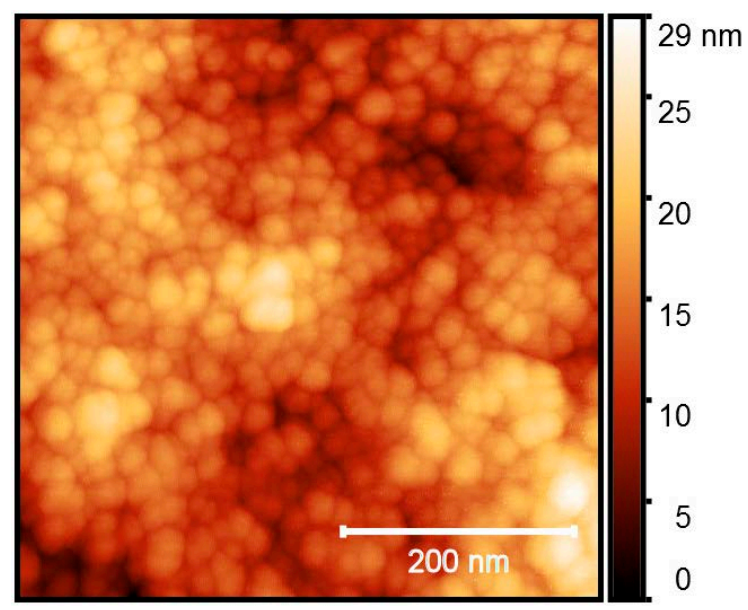

Fig. 4-9. AFM image of ZnO-NPs seed layer on the spray-coated GO layer on a Si wafer, produced by dip-coating in the sol-gel solution and annealed at $300{ }^{\circ} \mathrm{C}$ for $1 \mathrm{~min}$.

As mentioned before, due to the rate of combustion of the GO layer at $300^{\circ} \mathrm{C}$, annealing in air was limited to only $1 \mathrm{~min}$. This leads to non-merged small $\mathrm{ZnO}$ particles with smaller crystalline grain boundaries compared to the samples that were annealed for $10 \mathrm{~min}$. The SEM images in Fig. 4-10 show the ZnO-NP size and the crystal grain boundaries for different annealing durations of 1 and 10 min. The samples were prepared by dip-coating the substrates with the sol-gel 
solution on $\mathrm{Si} / \mathrm{GO}$ and Si-wafer substrates, followed by annealing in air at 300 ${ }^{\circ} \mathrm{C}$ for 1 and $10 \mathrm{~min}$, respectively.

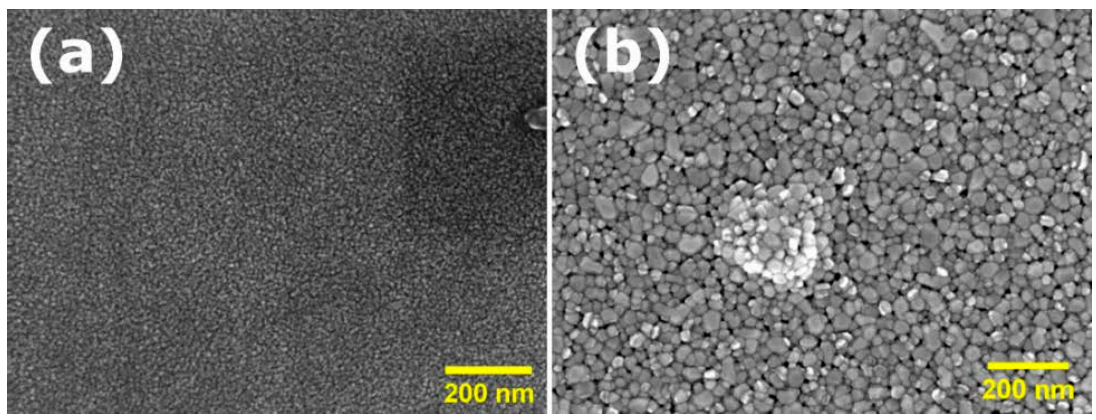

Fig. 4-10. SEM images of ZnO-NPs deposited by the dip-coating method and the sol-gel solution on (a) Si/GO and (b) Si-wafer substrates, followed by annealing in air at $300{ }^{\circ} \mathrm{C}$ for 1 and $10 \mathrm{~min}$, respectively.

\subsubsection{Reduced GO/Al-doped $\mathrm{ZnO}$ seed layers}

The thermal annealing [46] of the $\mathrm{Si} / \mathrm{GO} / \mathrm{ZnO}$ seed layers and exposure to UV irradiation [47] reduce GO to rGO. This GO-to-rGO transformation is along with a conductivity increasing and a transparency reduction. Higher conductivity of seed layers is important for electronic device fabrication, particularly on the insulating substrates. To obtain the maximum electrical conductivity, the ZnO NP seed layer was also highly doped with $\mathrm{Al}$ by adding $7.5 \mathrm{mM}$ aluminum nitrate into the sol-gel solution, explained in section 4.1.2, prior to the deposition on GO layers.

A minimum sheet resistivity of $8 \mathrm{~K} \Omega / \square$ was measured for an $\mathrm{rGO} / \mathrm{ZnO}$ :Al seed layer after annealing at $300{ }^{\circ} \mathrm{C}$ for $1 \mathrm{~min}$ and $10 \mathrm{~min} \mathrm{UV}$ irradiation. This conductive seed layer serves not only as a nucleation surface for the ZnO-NRs growth but also as a back contact to NRs, for later device fabrication.

\subsubsection{Distribution of polystyrene nanobeads on surfaces}

As explained in section 4.2.3, a suspension of PS-NBs was dispersed on the resist layer to define the opening areas in the CL technique. The PS-NBs are distributed on the surface due to the electrostatic net repulsive forces between the 
negatively charged particles. The radial distribution function (RDF) of the nanoholes was calculated based on SEM image analysis to analyze the particle distribution order.

For a 2D particle distribution, the $\mathrm{RDF}$ is defined as the ratio of particle surface density in an infinitesimal ring at a distance $r$ from a reference particle to the average particle surface density when all particles are uniformly distributed. Based on Fig. 4-11, the RDF is calculated according to equation (4.1) or equivalently (4.2), where $\mathrm{dn}(\mathrm{r})$ is the number of particles at a distance between $r$ and $r+d r, \rho$ is the average particle surface density $(=N / A, N$ is the total number of the particles in the entire area of interest), and $g(r)$ is the $\mathrm{RDF}[86,87]$.

The calculated $\mathrm{g}(\mathrm{r})$ values are independent of the original reference point and can have any value equal to or greater than zero. A $g(r)$ value greater than one indicates higher particle surface density than the average density $(\rho)$, meaning a dominant spacing between the neighboring particles.

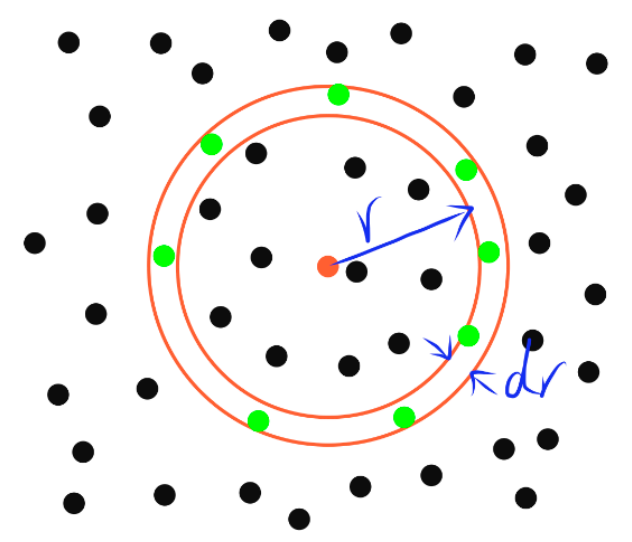

Fig. 4-11. A 2D particle distribution scheme.

$$
\begin{aligned}
& g(r)=\frac{(d n(r) / N)}{(d A(r) / A)} \\
& d n(r)=2 \pi r \rho g(r) d r
\end{aligned}
$$

The open-source ImageJ software and the additional incorporative Radial Distribution Function macro were used to calculate the $\mathrm{g}(\mathrm{r})$ functions. The 
calculation is based on the autocorrelation of the particle-center positions with respect to the various original preference points [88]. Using this algorithm and the fast Fourier transform (FFT), repeated patterns in the interparticle spacing can be identified from an apparently random distribution.

An example of using the RDF method for pattern-ordering analysis of an array of particles is shown in Fig. 4-12, compared with other alternative methods of 2D-FFT and the spatial distribution function (SDF) [88]. A specific amount of noise in terms of the interparticle spacing and particle dimension has been added to a computer-generated hexagonal array of particles with an interparticle spacing of 100 a.u., converting it to an apparently disordered and random particle distribution. Then, the obscured ordering in the structure has been retrieved using the described image analysis methods, as shown in Fig. 4-12(b-d).

Each peak in the RDF, or the intense bright points in the DSF images, represents the degree of periodicity in the structure. Samples with several RDF peaks have long-range ordering, while fewer peak numbers in the RDF indicate a short-range ordering structure. Our PS-NBs distributions show only one primary peak in their RDF graphs, meaning one period ordering between only the first neighboring particles.
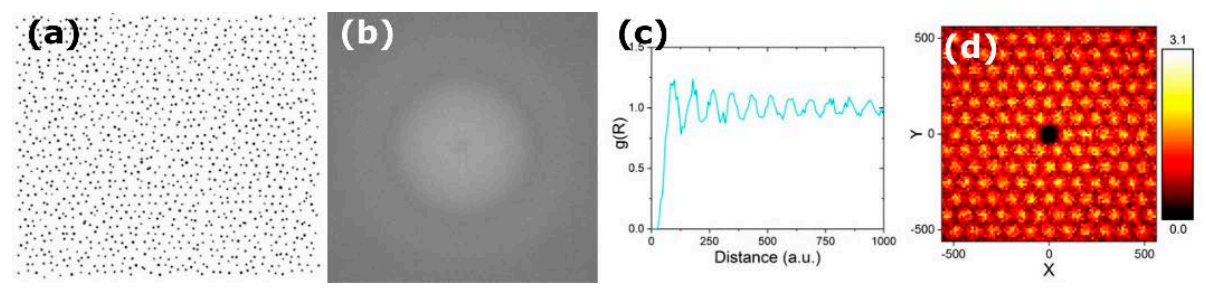

Fig. 4-12. (a) A computer-generated hexagonal array of particles with specific added noises of $20 \%$ interparticle spacing and $15 \%$ particle dimension. (b) 2D-FFT, (c) the RDF, and (d) the spatial distribution function (SDF) of the particles array in (a). Adapted with permission from [88]. Copyright 2019, American Chemical Society. 



\section{Conclusions and Outlook}

This research work started by exploring potentially 3D-printable conductive materials with maximum porosity for electronics. Among the possible candidates, $\mathrm{ZnO} /$ GR nanocomposites were selected based on their promising electrical and optical properties, and also because of the accumulated knowledge and experiences for working with $\mathrm{ZnO}$ in our research group.

Using a simple and inexpensive solution-based growth technique, several ZnO-GR nanocomposites with and without doping in various morphologies were successfully grown and characterized with respect to electrical conductivity, dispersity, bonding quality between the species, and optical properties. Our results demonstrate the dependence of the electrical and optical characteristics of the nanocomposites on the growth parameters, like the $\mathrm{pH}$ value.

Optical characterization of the developed composites showed an effective charge separation at the $\mathrm{ZnO}-\mathrm{GR}$ interface, which results in a longer lifetime of the photogenerated charge carriers and enhanced persistent photoconductivity. In addition, the growth of ZnO-NRs on the GR surface introduced effective spacers, leading to a high porosity in the final structure and significantly promoted GR wettability, essential for integrating the nanocomposite with other materials such as polymers for 3D printing.

The observed charge separation mechanism encouraged us to investigate the photocatalytic performance of our materials. The $\mathrm{ZnO}-\mathrm{GR}$ nanocomposites were sensitized to visible light by adding a low bandgap AgI semiconductor to the composite. A significantly enhanced solar-driven photocatalytic performance was observed in the final optimized $\mathrm{ZnO} / \mathrm{GR} / \mathrm{Ag} / \mathrm{AgI}$ nanocomposites due to the increased visible light absorption by AgI, and efficient charge separation of photogenerated carriers at the $\mathrm{ZnO}-\mathrm{GR}$ interface. 
To precisely control the growth area, density, and alignment of NRs for advanced electronics and photonics, a template-assisted selective area growth method was developed to synthesize arrays of single vertically aligned ZnO-NRs on Si or glass substrates. This technique is based on colloidal lithography patterning and preparing a compact and uniform $\mathrm{ZnO}$ seed layer by a sol-gel and dip-coating process. Compared to the ZnO-NRs grown on non-patterned substrates, it was shown that much better aligned NRs, with a tunable diameter and density, can be achieved by our method. However, a (100)-oriented Si substrate is not the preferred choice of crystalline substrate for vertical $\mathrm{ZnO}-\mathrm{NR}$ growth. We developed a novel route to mitigate this problem in Paper IV, where a GO buffer layer was introduced between the substrate and the $\mathrm{ZnO}$ seed layer. A significant improvement in the $\mathrm{ZnO}-\mathrm{NR}$ growth was verified using a non-crystalline $\mathrm{ZnO}$ seed layer on the partially reduced GO (rGO) buffer layer, together with the previous patterning technique.

A considerable effort was put into realizing a method to produce ordered arrays of vertical ZnO-NRs. Due to time constraints, the final optimized aligned structures were not yet tested for exciting applications, e.g., biosensors, LEDs, or field-emission devices. Due to thermal and oxidation stability, high-aspect ratio, and controllable length and diameter of the aligned $\mathrm{ZnO}-\mathrm{NRs}$ on conductive $\mathrm{rGO} / \mathrm{ZnO} \mathrm{Al}$ layers, a great potential of these structures is foreseen in all of the abovementioned applications.

Despite the improvement in vertical alignment of the $\mathrm{ZnO}-\mathrm{NRs}$ using the CL-patterning technique, the nanoholes' exact position is not controllable. To achieve such a control, precise nanoimprint lithography (NIL) can be employed to create the desired patterns on the $\mathrm{rGO} / \mathrm{ZnO}$ seed layers. A more systematic study is required to optimize the parameters and realize the nanohole patterns with specific diameter and pitch. Further research, based on this thesis work deals with developing novel spray-coated conductive and transparent rGO top contacts for realizing disruptive ZnO NR-based optoelectronics. 


\section{References}

1. Fan W, Zhang Q, Wang Y (2013) Semiconductor-based nanocomposites for photocatalytic $\mathrm{H} 2$ production and CO 2 conversion. Physical Chemistry Chemical Physics 15:2632-2649. https://doi.org/10.1039/C2CP43524A

2. Wang F, Li Q, Xu D (2017) Recent Progress in Semiconductor-Based Nanocomposite Photocatalysts for Solar-to-Chemical Energy Conversion. Advanced $\quad$ Energy $\quad$ Materials https://doi.org/10.1002/aenm.201700529

3. Kango S, Kalia S, Celli A, et al (2013) Surface modification of inorganic nanoparticles for development of organic-inorganic nanocomposites-A review. Progress in Polymer Science 38:1232-1261. https://doi.org/10.1016/j.progpolymsci.2013.02.003

4. Gao N, Fang X (2015) Synthesis and Development of Graphene-Inorganic Semiconductor Nanocomposites. Chem Rev 115:8294-8343. https://doi.org/10.1021/cr400607y

5. Kim Y-J, Lee J-H, Yi G-C (2009) Vertically aligned ZnO nanostructures grown on graphene layers. Applied Physics Letters 95:213101. https://doi.org/10.1063/1.3266836

6. Lee JM, Pyun YB, Yi J, et al (2009) ZnO Nanorod-Graphene Hybrid Architectures for Multifunctional Conductors. J Phys Chem C 113:1913419138. https://doi.org/10.1021/jp9078713

7. Lin J, Penchev M, Wang G, et al (2010) Heterogeneous Graphene Nanostructures: ZnO Nanostructures Grown on Large-Area Graphene Layers. Small 6:2448-2452. https://doi.org/10.1002/smll.201000250

8. Kim Y-J, Hadiyawarman, Yoon A, et al (2011) Hydrothermally grown ZnO nanostructures on few-layer graphene sheets. Nanotechnology 22:245603. https://doi.org/10.1088/0957-4484/22/24/245603

9. Chandraiahgari CR, Bellis GD, Balijepalli SK, et al (2016) Control of the size and density of $\mathrm{ZnO}$-nanorods grown onto graphene nanoplatelets in 
aqueous suspensions. RSC Adv 6:83217-83225.

https://doi.org/10.1039/C6RA18317D

10. Munshi AM, Dheeraj DL, Fauske VT, et al (2012) Vertically Aligned GaAs Nanowires on Graphite and Few-Layer Graphene: Generic Model and Epitaxial Growth. Nano Lett 12:4570-4576. https://doi.org/10.1021/nl3018115

11. Hwang JO, Lee DH, Kim JY, et al (2011) Vertical ZnO nanowires/graphene hybrids for transparent and flexible field emission. J Mater Chem 21:3432-3437. https://doi.org/10.1039/C0JM01495H

12. Di Bartolomeo A (2016) Graphene Schottky diodes: An experimental review of the rectifying graphene/semiconductor heterojunction. Physics Reports 606:1-58. https://doi.org/10.1016/j.physrep.2015.10.003

13. Ertekin E, Greaney PA, Sands TD, Chrzan DC (2002) Equilibrium Analysis of Lattice-Mismatched Nanowire Heterostructures. MRS Online Proceedings Library Archive 737:. https://doi.org/10.1557/PROC-737-F10.4

14. Sandiumenge F (2017) Strain landscapes and self-organization of free surfaces in complex oxide epitaxy. Journal of Materials Research 32:39583976. https://doi.org/10.1557/jmr.2017.317

15. Kołodziejczak-Radzimska A, Jesionowski T (2014) Zinc Oxide-From Synthesis to Application: A Review. Materials 7:2833-2881. https://doi.org/10.3390/ma7042833

16. Klingshirn C, Fallert J, Zhou H, et al (2010) 65 years of ZnO research old and very recent results. phys stat sol (b) 247:1424-1447. https://doi.org/10.1002/pssb.200983195

17. Janotti A, Van de Walle CG (2006) New insights into the role of native point defects in ZnO. Journal of Crystal Growth 287:58-65. https://doi.org/10.1016/j.jcrysgro.2005.10.043

18. Janotti A, Van de Walle CG (2007) Native point defects in ZnO. Phys Rev B 76:165202. https://doi.org/10.1103/PhysRevB.76.165202

19. Janotti A, Walle CGV de (2009) Fundamentals of zinc oxide as a semiconductor. Rep Prog Phys 72:126501. https://doi.org/10.1088/00344885/72/12/126501

20. Choi S, Phillips MR, Aharonovich I, et al (2015) Photophysics of Point Defects in ZnO Nanoparticles. Advanced Optical Materials 3:821-827. https://doi.org/10.1002/adom.201400592 
21. Galdámez-Martinez A, Santana G, Güell F, et al (2020) Photoluminescence of $\mathrm{ZnO}$ Nanowires: A Review. Nanomaterials 10:857. https://doi.org/10.3390/nano10050857

22. Liu R, Chen Y, Ding S, et al (2019) Preparation of highly transparent conductive aluminum-doped zinc oxide thin films using a low-temperature aqueous solution process for thin-film solar cells applications. Solar Energy Materials and Solar Cells 203:110161. https://doi.org/10.1016/j.solmat.2019.110161

23. Hsu C-H, Chen D-H (2010) Synthesis and conductivity enhancement of Al-doped ZnO nanorod array thin films. Nanotechnology 21:285603. https://doi.org/10.1088/0957-4484/21/28/285603

24. Hagendorfer H, Lienau K, Nishiwaki S, et al (2014) Highly Transparent and Conductive ZnO: Al Thin Films from a Low Temperature Aqueous Solution Approach. Adv Mater 26:632-636. https://doi.org/10.1002/adma.201303186

25. Bamiduro O, Mustafa H, Mundle R, et al (2007) Metal-like conductivity in transparent Al:ZnO films. Appl Phys Lett 90:252108. https://doi.org/10.1063/1.2749836

26. Nian Q, Callahan M, Look D, et al (2015) Highly transparent conductive electrode with ultra-low HAZE by grain boundary modification of aqueous solution fabricated alumina-doped zinc oxide nanocrystals. APL Materials 3:062803. https://doi.org/10.1063/1.4915489

27. Jia J, Takasaki A, Oka N, Shigesato Y (2012) Experimental observation on the Fermi level shift in polycrystalline Al-doped $\mathrm{ZnO}$ films. Journal of Applied Physics 112:013718. https://doi.org/10.1063/1.4733969

28. Consonni V, Lord AM (2021) Polarity in $\mathrm{ZnO}$ nanowires: A critical issue for piezotronic and piezoelectric devices. Nano Energy 83:105789. https://doi.org/10.1016/j.nanoen.2021.105789

29. Song Y, Wang N, Fadlallah MM, et al (2021) Defect states contributed nanoscale contact electrification at $\mathrm{ZnO}$ nanowires packed film surfaces. Nano Energy 79:105406. https://doi.org/10.1016/j.nanoen.2020.105406

30. Azulay AR, Turkulets Y, Gaudio DD, et al (2020) Why do nanowires grow with their c-axis vertically-aligned in the absence of epitaxy? Scientific Reports 10:6554. https://doi.org/10.1038/s41598-020-63500-y

31. Cossuet T, Roussel H, Chauveau J-M, et al (2018) Well-ordered ZnO nanowires with controllable inclination on semipolar $\mathrm{ZnO}$ surfaces by 
chemical bath deposition. Nanotechnology 29:475601. https://doi.org/10.1088/1361-6528/aadf62

32. Mitra P, Chatterjee AP, Maiti HS (1998) ZnO thin film sensor. Materials Letters 35:33-38. https://doi.org/10.1016/S0167-577X(97)00215-2

33. Zhu Q, Xie C, Li H, Zeng D (2016) A method for modeling and deciphering the persistent photoconductance and long-term charge storage of $\mathrm{ZnO}$ nanorod arrays. Nano Res 9:2972-3002. https://doi.org/10.1007/s12274016-1182-y

34. Wu XL, Siu GG, Fu CL, Ong HC (2001) Photoluminescence and cathodoluminescence studies of stoichiometric and oxygen-deficient $\mathrm{ZnO}$ films. Appl Phys Lett 78:2285-2287. https://doi.org/10.1063/1.1361288

35. Zhu Q, Xie C, Li H, et al (2015) Through-Process Analytical Modeling of Photoconductance Spectrum for Porous ZnO Nanocrystalline Film. Chem Mater 27:2861-2874. https://doi.org/10.1021/cm504739f

36. Moore JC, Thompson CV (2013) A Phenomenological Model for the Photocurrent Transient Relaxation Observed in $\mathrm{ZnO}$-Based Photodetector Devices. Sensors (Basel) 13:9921-9940. https://doi.org/10.3390/s130809921

37. Edinger S, Bansal N, Bauch M, et al (2017) Comparison of chemical bathdeposited ZnO films doped with Al, Ga and In. J Mater Sci 52:9410-9423. https://doi.org/10.1007/s10853-017-1104-8

38. Weiss NO, Zhou H, Liao L, et al (2012) Graphene: An Emerging Electronic Material. Adv Mater 24:5782-5825. https://doi.org/10.1002/adma.201201482

39. (2017) Nanotechnologies-Vocabulary-Part 13: Graphene and Related Two-Dimensional (2D) Materials

40. Pollard AJ, Paton KR, Clifford CA, Legge E (2017) Characterisation of the structure of graphene

41. Gee C-M, Tseng C-C, Wu F-Y, et al (2013) Flexible transparent electrodes made of electrochemically exfoliated graphene sheets from low-cost graphite pieces. Displays 34:315-319. https://doi.org/10.1016/j.displa.2012.11.002

42. Arapov K, Rubingh E, Abbel R, et al (2016) Conductive Screen Printing Inks by Gelation of Graphene Dispersions. Adv Funct Mater 26:586-593. https://doi.org/10.1002/adfm.201504030 
43. Yu H, Zhang B, Bulin C, et al (2016) High-efficient Synthesis of Graphene Oxide Based on Improved Hummers Method. Scientific Reports 6:36143. https://doi.org/10.1038/srep36143

44. Chen J, Yao B, Li C, Shi G (2013) An improved Hummers method for ecofriendly synthesis of graphene oxide. Carbon 64:225-229. https://doi.org/10.1016/j.carbon.2013.07.055

45. Marcano DC, Kosynkin DV, Berlin JM, et al (2010) Improved Synthesis of Graphene Oxide. ACS Nano 4:4806-4814. https://doi.org/10.1021/nn1006368

46. Sengupta I, Chakraborty S, Talukdar M, et al (2018) Thermal reduction of graphene oxide: How temperature influences purity. Journal of Materials Research 33:4113-4122. https://doi.org/10.1557/jmr.2018.338

47. Ji T, Hua Y, Sun M, Ma N (2013) The mechanism of the reaction of graphite oxide to reduced graphene oxide under ultraviolet irradiation. Carbon 54:412-418. https://doi.org/10.1016/j.carbon.2012.11.057

48. Jariwala D, Marks TJ, Hersam MC (2017) Mixed-dimensional van der Waals heterostructures. Nature Materials 16:170-181. https://doi.org/10.1038/nmat4703

49. Choi WM, Shin K-S, Lee HS, et al (2011) Selective growth of ZnO nanorods on $\mathrm{SiO} 2 / \mathrm{Si}$ substrates using a graphene buffer layer. Nano Res 4:440-447. https://doi.org/10.1007/s12274-011-0100-6

50. Larson K, Clark A, Appel A, et al (2015) Surface-dependence of interfacial binding strength between zinc oxide and graphene. RSC Advances 5:65719-65724. https://doi.org/10.1039/C5RA13048D

51. Müller R, Huber F, Gelme O, et al (2019) Chemical Vapor Deposition Growth of Zinc Oxide on Sapphire with Methane: Initial Crystal Formation Process. Crystal Growth \& Design 19:4964-4969. https://doi.org/10.1021/acs.cgd.9b00181

52. Gomez JL, Tigli O (2013) Zinc oxide nanostructures: from growth to application. J Mater Sci 48:612-624. https://doi.org/10.1007/s10853-0126938-5

53. Rahman F (2019) Zinc oxide light-emitting diodes: a review. OE 58:010901. https://doi.org/10.1117/1.OE.58.1.010901

54. Gardeniers JGE, Rittersma ZM, Burger GJ (1998) Preferred orientation and piezoelectricity in sputtered $\mathrm{ZnO}$ films. Journal of Applied Physics 83:7844-7854. https://doi.org/10.1063/1.367959 
55. Bacaksiz E, Parlak M, Tomakin M, et al (2008) The effects of zinc nitrate, zinc acetate and zinc chloride precursors on investigation of structural and optical properties of $\mathrm{ZnO}$ thin films. Journal of Alloys and Compounds 466:447-450. https://doi.org/10.1016/j.jallcom.2007.11.061

56. Yun S, Lee J, Yang J, Lim S (2010) Hydrothermal synthesis of Al-doped $\mathrm{ZnO}$ nanorod arrays on $\mathrm{Si}$ substrate. Physica B: Condensed Matter 405:413-419. https://doi.org/10.1016/j.physb.2009.08.297

57. Gao Y, Nagai M (2006) Morphology Evolution of ZnO Thin Films from Aqueous Solutions and Their Application to Solar Cells. Langmuir 22:3936-3940. https://doi.org/10.1021/la053042f

58. Vayssieres L (2003) Growth of Arrayed Nanorods and Nanowires of ZnO from Aqueous Solutions. Adv Mater 15:464-466. https://doi.org/10.1002/adma.200390108

59. Sakai D, Nagashima K, Yoshida H, et al (2019) Substantial Narrowing on the Width of "Concentration Window" of Hydrothermal ZnO Nanowires via Ammonia Addition. Sci Rep 9:1-10. https://doi.org/10.1038/s41598019-50641-y

60. Chalangar E, Machhadani H, Lim S-H, et al (2018) Influence of morphology on electrical and optical properties of graphene/Al-doped $\mathrm{ZnO}$-nanorod composites. Nanotechnology 29:415201. https://doi.org/10.1088/1361-6528/aad3ec

61. Fuchs P, Hagendorfer H, Romanyuk YE, Tiwari AN (2015) Doping strategies for highly conductive $\mathrm{Al}$-doped $\mathrm{ZnO}$ films grown from aqueous solution. Phys Status Solidi A 212:51-55. https://doi.org/10.1002/pssa.201431145

62. Verrier C, Appert E, Chaix-Pluchery O, et al (2017) Effects of the pH on the Formation and Doping Mechanisms of $\mathrm{ZnO}$ Nanowires Using Aluminum Nitrate and Ammonia. Inorg Chem 56:13111-13122. https://doi.org/10.1021/acs.inorgchem.7b01916

63. Gfroerer TH (2006) Photoluminescence in Analysis of Surfaces and Interfaces. In: Encyclopedia of Analytical Chemistry. John Wiley \& Sons, Ltd

64. Smits FM (1958) Measurement of Sheet Resistivities with the Four-Point Probe. Bell System Technical Journal 37:711-718. https://doi.org/10.1002/j.1538-7305.1958.tb03883.x

65. Rani M, Tripathi SK (2016) Electron transfer properties of organic dye sensitized $\mathrm{ZnO}$ and $\mathrm{ZnO} / \mathrm{TiO} 2$ photoanode for dye sensitized solar cells. 
Renewable and Sustainable Energy Reviews 61:97-107. https://doi.org/10.1016/j.rser.2016.03.012

66. Xu S, Wang ZL (2011) One-dimensional ZnO nanostructures: Solution growth and functional properties. Nano Res 4:1013-1098. https://doi.org/10.1007/s12274-011-0160-7

67. Li Y, Li X, Li J, Yin J (2006) Photocatalytic degradation of methyl orange by TiO2-coated activated carbon and kinetic study. Water Research 40:1119-1126. https://doi.org/10.1016/j.watres.2005.12.042

68. Zhu Q, Xie C, Li H, et al (2014) Selectively enhanced UV and NIR photoluminescence from a degenerate $\mathrm{ZnO}$ nanorod array film. Journal of Materials Chemistry C 2:4566-4580. https://doi.org/10.1039/C4TC00011K

69. Rahman MM, Khan MKR, Islam MR, et al (2012) Effect of Al Doping on Structural, Electrical, Optical and Photoluminescence Properties of NanoStructural ZnO Thin Films. Journal of Materials Science \& Technology 28:329-335. https://doi.org/10.1016/S1005-0302(12)60064-4

70. Chalangar E (2019) Graphene-based nanocomposites for electronics and photocatalysis. Linköping University Electronic Press, Linköping

71. Li G, Wang Y, Mao L (2014) Recent progress in highly efficient Ag-based visible-light photocatalysts. RSC Adv 4:53649-53661. https://doi.org/10.1039/C4RA08044K

72. Kaspar TC, Droubay T, Chambers SA, Bagus PS (2010) Spectroscopic Evidence for Ag(III) in Highly Oxidized Silver Films by X-ray Photoelectron Spectroscopy. J Phys Chem C 114:21562-21571. https://doi.org/10.1021/jp107914e

73. Adam RE, Chalangar E, Pirhashemi M, et al (2019) Graphene-based plasmonic nanocomposites for highly enhanced solar-driven photocatalytic activities. $\quad$ RSC Adv 9:30585-30598. https://doi.org/10.1039/C9RA06273D

74. Wissler M (2006) Graphite and carbon powders for electrochemical applications. Journal of Power Sources 156:142-150. https://doi.org/10.1016/j.jpowsour.2006.02.064

75. Parize R, Garnier JD, Appert E, et al (2018) Effects of Polyethylenimine and Its Molecular Weight on the Chemical Bath Deposition of ZnO Nanowires. ACS Omega 3:12457-12464. https://doi.org/10.1021/acsomega.8b01641 
76. Verrier C, Appert E, Chaix-Pluchery O, et al (2017) Tunable Morphology and Doping of ZnO Nanowires by Chemical Bath Deposition Using Aluminum Nitrate. J Phys Chem C 121:3573-3583. https://doi.org/10.1021/acs.jpcc.6b11104

77. Xu S, Wei Y, Kirkham M, et al (2008) Patterned Growth of Vertically Aligned $\mathrm{ZnO}$ Nanowire Arrays on Inorganic Substrates at Low Temperature without Catalyst. J Am Chem Soc 130:14958-14959. https://doi.org/10.1021/ja806952j

78. Fredriksson H, Alaverdyan Y, Dmitriev A, et al (2007) Hole-Mask Colloidal Lithography. Advanced Materials 19:4297-4302. https://doi.org/10.1002/adma.200700680

79. Hanarp P, Sutherland D, Gold J, Kasemo B (1999) Nanostructured model biomaterial surfaces prepared by colloidal lithography. Nanostructured Materials 12:429-432. https://doi.org/10.1016/S0965-9773(99)001518

80. Cesaria M, Taurino A, Manera MG, et al (2019) Gold nanoholes fabricated by colloidal lithography: novel insights into nanofabrication, short-range correlation and optical properties. Nanoscale 11:8416-8432. https://doi.org/10.1039/C8NR09911A

81. Chalangar E, Nur O, Willander M, et al (2021) Synthesis of Vertically Aligned ZnO Nanorods Using Sol-gel Seeding and Colloidal Lithography Patterning. Nanoscale Research Letters 16:46. https://doi.org/10.1186/s11671-021-03500-7

82. Vellekoop MJ, Visser CCO, Sarro PM, Venema A (1990) Compatibility of zinc oxide with silicon IC processing. Sensors and Actuators A: Physical 23:1027-1030. https://doi.org/10.1016/0924-4247(90)87083-U

83. Meyer E, Hug HJ, Bennewitz R (2004) Scanning Probe Microscopy: The Lab on a Tip. Springer-Verlag, Berlin Heidelberg

84. Kumar M, Kanjilal A, Som T (2013) Effect of grain-boundaries on electrical properties of $\mathrm{n}-\mathrm{ZnO}: \mathrm{Al} / \mathrm{p}-\mathrm{Si}$ heterojunction diodes. AIP Advances 3:092126. https://doi.org/10.1063/1.4823480

85. Bandyopadhyay S, Paul GK, Roy R, et al (2002) Study of structural and electrical properties of grain-boundary modified $\mathrm{ZnO}$ films prepared by sol-gel technique. Materials Chemistry and Physics 74:83-91. https://doi.org/10.1016/S0254-0584(01)00402-3

86. Younge K, Johnston B, Christenson C, et al (2006) The use of radial distribution and pair-correlation functions to analyze and describe biological 
aggregations. Limnology and Oceanography: Methods 4:382-391. https://doi.org/10.4319/lom.2006.4.382

87. Thorneywork AL, Roth R, Aarts DGAL, Dullens RPA (2014) Communication: Radial distribution functions in a two-dimensional binary colloidal hard sphere system. J Chem Phys 140:161106. https://doi.org/10.1063/1.4872365

88. Mac Fhionnlaoich N, Qi R, Guldin S (2019) Application of the Spatial Distribution Function to Colloidal Ordering. Langmuir 35:16605-16611. https://doi.org/10.1021/acs.langmuir.9b02877 

Part II: Papers 
PAPER

I 


\title{
Influence of morphology on electrical and optical properties of graphene/Al-doped ZnO-nanorod composites
}

\author{
Ebrahim Chalangar ${ }^{1,2} \oplus$, Houssaine Machhadani ${ }^{3}$, Seung-Hyuk Lim $^{3}$, \\ K Fredrik Karlsson ${ }^{3}$, Omer Nur ${ }^{1} \oplus$, Magnus Willander $^{1}$ and \\ Håkan Pettersson ${ }^{1,2,4}$ \\ ${ }^{1}$ Department of Science and Technology (ITN), Faculty of Science \& Engineering, Linköping University, \\ Norrköping, Sweden \\ ${ }^{2}$ Department of Mathematics, Physics and Electrical Engineering, Halmstad University, Halmstad, Sweden \\ ${ }^{3}$ Semiconductor Materials, Department of Physics, Chemistry, and Biology (IFM), Linköping University, \\ Linköping, Sweden \\ ${ }^{4}$ Solid State Physics and NanoLund, Lund University, Lund, Sweden
}

E-mail: hakan.pettersson@hh.se

Received 15 May 2018, revised 26 June 2018

Accepted for publication 17 July 2018

Published 1 August 2018

\section{Abstract}

The development of future 3D-printed electronics relies on the access to highly conductive inexpensive materials that are printable at low temperatures $\left(<100^{\circ} \mathrm{C}\right)$. The implementation of available materials for these applications are, however, still limited by issues related to cost and printing quality. Here, we report on the simple hydrothermal growth of novel nanocomposites that are well suited for conductive printing applications. The nanocomposites comprise highly $\mathrm{Al}$-doped $\mathrm{ZnO}$ nanorods grown on graphene nanoplatelets (GNPs). The $\mathrm{ZnO}$ nanorods play the two major roles of (i) preventing GNPs from agglomerating and (ii) promoting electrical conduction paths between the graphene platelets. The effect of two different $\mathrm{ZnO}$-nanorod morphologies with varying Al-doping concentration on the nanocomposite conductivity and the graphene dispersity are investigated. Time-dependent absorption, photoluminescence and photoconductivity measurements show that growth in high $\mathrm{pH}$ solutions promotes a better graphene dispersity, higher doping levels and enhanced bonding between the graphene and the $\mathrm{ZnO}$ nanorods. Growth in low $\mathrm{pH}$ solutions yields samples characterized by a higher conductivity and a reduced number of surface defects. These samples also exhibit a large persistent photoconductivity attributed to an effective charge separation and transfer from the nanorods to the graphene platelets. Our findings can be used to tailor the conductivity of novel printable composites, or for fabrication of large volumes of inexpensive porous conjugated graphene-semiconductor composites.

Keywords: graphene, zinc oxide, nanorods, nanocomposites, persistent photoconductivity, printing

(Some figures may appear in colour only in the online journal)

\section{Introduction}

Original content from this work may be used under the terms of the Creative Commons Attribution 3.0 licence. Any further distribution of this work must maintain attribution to the author(s) and the title of the work, journal citation and DOI
The development of 3D-printing technologies for realizing electronic components and materials is evolving very quickly. Presently, there is a strong interest in developing novel 3D-printable 
functional nanocomposites for device fabrication. For instance, conductive nanocomposites can be optimized for low-temperature printing of conductors using the well-developed fused deposition modeling printers available on the market today [1-3] Carbon-based nanocomposite materials, containing graphene in particular, are inexpensive attractive candidates with remarkable physical properties that make them well suited for 3D-printing of electronics [4-6].

Demonstrated properties that make graphene promising for electronics include excellent (i) carrier mobility (up to $\left.10^{6} \mathrm{~cm}^{2} \mathrm{~V}^{-1} \mathrm{~s}^{-1}\right)$ [7], (ii) electrical resistivity $(0.1-6 \mathrm{k} \Omega / \square$ for a single layer with $97.7 \%$ optical transparency) [7], (iii) chemical stability and (iv) mechanical strength (double to that of CNTs) [7]. However, graphene is highly hydrophobic and hardly interacts with other materials. Moreover, the highaspect-ratio of graphene nanoplatelets (GNPs), combined with large van der Waals forces typically lead to issues with undesired agglomeration into large particles. In order to use graphene in printing applications, it must therefore be properly dispersed in a suitable medium. High-quality dispersions are typically facilitated by introducing chemical surfactants, [8] which unfortunately influence the electrical properties of graphene in an undesired way. A different approach to synthesize a conductive screen printing graphene ink with a resistance of $30 \Omega / \square$ at $25 \mu \mathrm{m}$ thickness by gelation of a graphene dispersion was recently reported [9]. Despite all of these efforts it still remains a challenge to make a highly conductive graphene dispersion in a viscous medium, suitable for printing applications.

Here we propose a new approach to realize a graphene dispersion, effectively forming a conductive network, by growing zinc oxide nanorods ( $\mathrm{ZnO}-\mathrm{NRs}$ ), degenerately doped with aluminum (Al), as highly conductive spacers on the surface of the GNPs. The potential advantages of these composites stem from the remarkable charge transport characteristics of graphene, combined with the excellent optical characteristics of $\mathrm{ZnO}$. The expected enhancement of the optoelectronic properties of this composite might lead to important applications in optoelectronics, photovoltaics and photocatalytics [10].

Zinc oxide is a well-known unintentionally n-type semiconductor with a wide direct bandgap of $3.2-3.4 \mathrm{eV}$ at roomtemperature. It can be grown in different nanostructured shapes e.g. nanorods by inexpensive low-temperature $\left(<100^{\circ} \mathrm{C}\right)$ hydrothermal solution-based procedures $[11,12]$. The morphology, length and diameter of the $\mathrm{ZnO}-\mathrm{NRs}$ can be controlled by tuning the $\mathrm{pH}$ value, temperature of the growth solution, precursor concentration and growth duration [13].

ZnO-NRs can be grown on GNPs with excellent result despite of their hydrophobicity. It has been shown that the reduced lattice mismatch of less than $2 \%[14,15]$ between ZnO-NRs and GNPs causes strong atomic level interactions which lead to strong bonding and high mechanical stability [16]. Moreover, the electrical interface between GNPs and ZnO-NRs shows excellent low-ohmic metallic behavior $[17,18]$ of great importance for conductive applications.

\section{Experimental details}

All the chemicals required for the hydrothermal growth presented in this work, including the multilayer GNP powder, were purchased from Sigma-Aldrich. The first step was to seed the GNPs with a layer of $\mathrm{ZnO}$ nanoparticles (ZnO-NPs) [19]. GNPs with a concentration of $0.5 \mathrm{~g} \mathrm{l}^{-1}$ were initially dispersed in DI-water using an ultrasonic bath for $10 \mathrm{~min}$. Subsequently this solution was mixed with a $100 \mathrm{ml}$ solution of $0.005 \mathrm{M}$ of zinc acetate $\left(\mathrm{Zn}\left(\mathrm{CH}_{3} \mathrm{COO}\right)_{2} .2 \mathrm{H}_{2} \mathrm{O}\right)$ and $0.025 \mathrm{M}$ of $\mathrm{KOH}$ in water at $60^{\circ} \mathrm{C}$ in an ultrasonic bath for $10 \mathrm{~min}$. The seeded GNPs were subsequently washed in water and centrifuged $(3000 \mathrm{rpm}, 10 \mathrm{~min})$ three times, followed by annealing in nitrogen at $300{ }^{\circ} \mathrm{C}$ for $30 \mathrm{~min}$ to enhance the attachment of the ZnO-NPs to the GNPs. For the growth of $\mathrm{ZnO}-\mathrm{NRs}$, we prepared two solutions of $0.025 \mathrm{M}$ of $\mathrm{Zn}\left(\mathrm{NO}_{3}\right)_{2} \cdot 6 \mathrm{H}_{2} \mathrm{O}$ and $0.025 \mathrm{M}$ of $\mathrm{C}_{6} \mathrm{H}_{12} \mathrm{~N}_{4}$ (HMT) in DI-water with $\mathrm{pH} 6.6$ and $\mathrm{pH} 11$ (by adding ammonia), respectively. These two different $\mathrm{pH}$ growth solutions resulted in significantly different $\mathrm{ZnO}-\mathrm{NRs}$ morphologies. A $0.002 \mathrm{M}$ aqueous solution of $\mathrm{Al}\left(\mathrm{NO}_{3}\right)_{3} \cdot 9 \mathrm{H}_{2} \mathrm{O}$ was stirred overnight and added to the growth solution to supply the $\mathrm{Al}$ for doping of the NRs. Finally the seeded GNPs were added to the growth solution under stirring for $2 \mathrm{~h}$ at $75^{\circ} \mathrm{C}$.

\section{Results and discussion}

Figure 1(a) shows a schematic of the grown nanostructures. The ZnO:Al-NRs play two major roles for the final composites. First, they make a physical spacer between the GNPs proportional to their length. These physical spacers prevent GNPs from agglomerating and make the structure more porous. The porosities can, depending on the application, be filled by a proper matrix material. Second, degenerately doped $\mathrm{ZnO}$-NRs grown on one GNP will form low-ohmic electrical connections to a neighboring GNP. Previous studies have shown that the formation of low-resistance direct contacts between the particles is the most crucial factor for realization of highly conductive polymer composites [20]. The sharp NRs can also more easily penetrate through any polymer matrix filling up the porosities between the GNPs and increase the probability of direct contact formation between GNPs. The overall electrical transport properties depend on the resulting complex conductive network between the GNPs in the matrix.

To make sure that the $\mathrm{ZnO}-\mathrm{NPs}$ in the seed layer are also doped with $\mathrm{Al}$, a piece of pure $\mathrm{Al}$ was inserted into the solution during the seeding of the GNPs. The high $\mathrm{pH}$ value of 13 of the seed solution will lead to a partially dissolving of $\mathrm{Al}$ in the solution and to doping of the ZnO-NPs [21]. Figure 1(b) shows GNPs decorated with ZnO:Al-NPs. The photoluminescence (PL) measurements discussed below clearly show the effects of the high Al-doping on the optical properties of the $\mathrm{ZnO}-\mathrm{NPs}$

The two different morphologies of $\mathrm{ZnO}-\mathrm{NRs}$ grown at different $\mathrm{pH}(6.6$ and 11) were investigated for better performance 

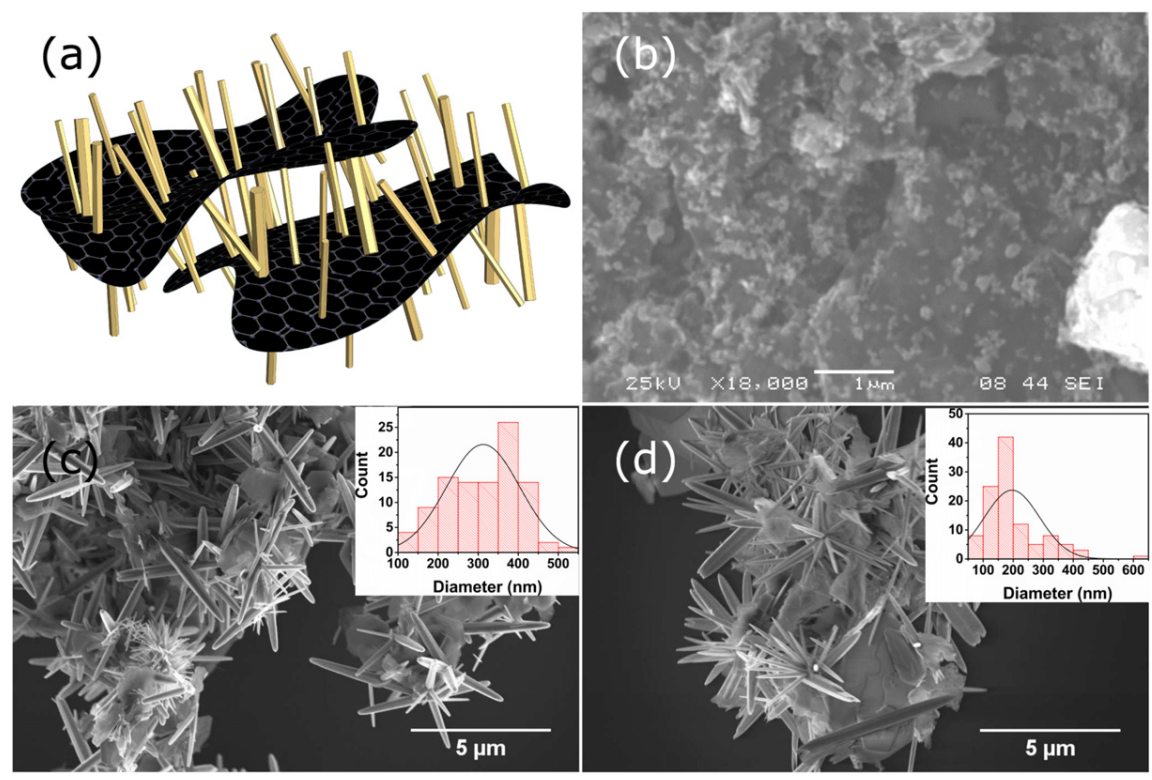

Figure 1. (a) Schematics of ZnO-NRs grown on both sides of GNPs by the hydrothermal solution-based procedure. (b) Scanning electron microscopy (SEM) image of GNPs decorated with Al-doped $\mathrm{ZnO}$ nanoparticles. (c) Thick hexagonal low-density $\mathrm{ZnO}-\mathrm{NRs}$ grown at $\mathrm{pH}$ 6.6. (d) Thin needle-like, high-density ZnO-NRs grown at $\mathrm{pH} 11$. The insets show the size distribution of the nanorods diameter.
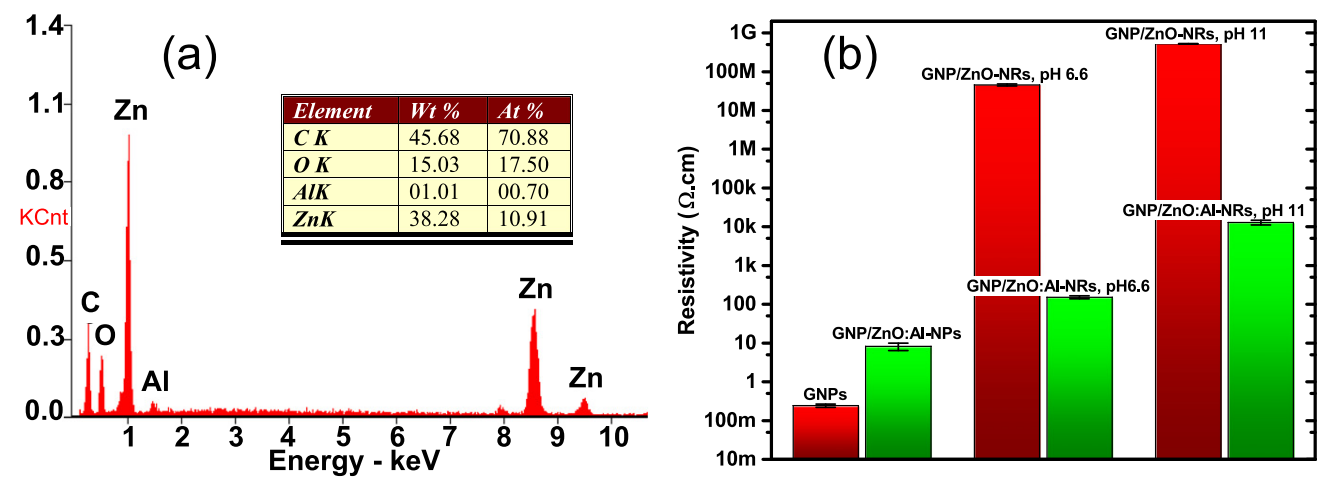

Figure 2. (a) Energy dispersive x-ray spectroscopy (EDS) spectra for GNP/ZnO:Al-NRs grown in pH 11. (b) Resistivity of GNPs, GNP/ ZnO:Al-NPs, GNP/ZnO-NRs (pH 6.6 and 11) and GNP/ZnO:Al-NRs (pH 6.6 and 11) measured by a four-point probe method.

regarding both conductivity and dispersity. $\mathrm{ZnO}-\mathrm{NRs}$ grown at $\mathrm{pH} 6.6$ (figure 1(c)) are hexagonal, thicker (mean diameter 313 $\mathrm{nm})$, longer $(1-2 \mu \mathrm{m})$ and more sparse compared to $\mathrm{ZnO}-\mathrm{NRs}$ grown at $\mathrm{pH} 11$. The NRs grown at high $\mathrm{pH}$ are more needle-like with a mean diameter and length of $196 \mathrm{~nm}$ and $0.5-1.5 \mu \mathrm{m}$ respectively (figure $1(\mathrm{~d})$ ). The density of $\mathrm{ZnO}-\mathrm{NRs}$ on the GNP surface was estimated, $1.4 \mu \mathrm{m}^{-2}$ for the samples grown at $\mathrm{pH} 6.6$ and $2.4 \mu \mathrm{m}^{-2}$ for the samples grown at $\mathrm{pH} 11$ by counting in the SEM image.
Figure 2(a) shows the abundance of different elements in the samples extracted from energy dispersive $\mathrm{x}$-ray spectroscopy (EDS). Carbon (C) is the most abundant element observed in all composites with an atomic ratio of 6.5 compared to $\mathrm{Zn}$. The presence of $0.5-1.5$ at\% $\mathrm{Al}$ is also detected in the doped composites, which basically represents the lower boundary for a typical EDS system. The doping concentration in the samples is estimated in the range of $0.7-1.2$ at $\%$ based on the dopant concentration in the growth solution $[22,23]$. The presence of 
oxygen $(\mathrm{O})$ is almost 60 at $\%$ higher than $\mathrm{Zn}$ in the samples. This excess of $\mathrm{O}$ could be related to the hydroxyl groups $\mathrm{OH}^{-}$and/or carboxyl groups $-\mathrm{COOH}$, or to adsorbed water on the GNPs or $\mathrm{ZnO}$ surfaces during the synthesis [24].

The resistivity of the composite layer samples, comprising the investigated microparticles, is measured by a standard fourpoint method. To do that, the layered materials must first be smoothly deposited. To deposit the randomized GNP/ZnO:AlNRs microparticles, we used a vacuum filtration method [8]. A water dispersion containing the microparticles was filtered by an Anodisc membrane filter from Whatman $(25 \mathrm{~mm}$ diameter, $0.2 \mu \mathrm{m}$ poresize) and dried at $70^{\circ} \mathrm{C}$ in an oven. The layer thickness, varying from 20 to $50 \mu \mathrm{m}$, was measured by a contact profilometer. As shown in figure 2(b), the resistivity of pure GNPs is the lowest and it increases after decoration with $\mathrm{ZnO}$ : Al-NPs. This increase can be explained by the synthesis procedure and/or the spacing induced by the doped $\mathrm{ZnO}$ nanoparticles. Growth of undoped $\mathrm{ZnO}-\mathrm{NRs}$ on the decorated GNPs results in a further increase of the resistivity for both investigated morphologies, effectively converting the layers to insulators. This high resistivity shows that the GNPs are physically separated from each other by the non-intentionally doped ZnO-NRs. For the doped samples, the resistivity strongly decreased and the nanocomposites again became conductive due to the enhanced conductivity of the degenerately doped ZnO:Al-NRs. This shows that the electrical current is passing through the $\mathrm{ZnO}$ NRs. The ZnO-NRs (nanoneedles) grown at $\mathrm{pH} 11$ show a comparably higher resistivity compared to those grown at $\mathrm{pH}$ 6.6. This suggests that nanoneedle-shaped GNPs are better dispersed. This hypothesis is supported by the optical UV-vis data discussed below.

To compare the dispersity of the two morphologies, and the attachment of $\mathrm{ZnO}-\mathrm{NRs}$ to the GNPs, time-dependent UV-vis spectroscopy was employed. Figures 3(a)-(c) show the absorption spectra of the GNPs with different morphologies of grown $\mathrm{ZnO}$ :Al-NRs, dispersed in isopropanol, after every $5 \mathrm{~min}$ and after $24 \mathrm{~h}$. The absorption spectra of pure GNPs exhibit a rather flat absorption characteristics dominated by spectral features beyond $1500 \mathrm{~nm}$ related to hydro$\mathrm{xyl}$ and/or carboxyl groups on the GNP surfaces (figure 3(a)). Figures 3(b) and (c) display a strong absorption peak at about $380 \mathrm{~nm}$ for dispersions of GNP/ZnO:Al-NRs due to the $\mathrm{ZnO}$ bandgap. The broad absorption shoulder, and spectrally unresolved absorption edge, reflect various scattering mechanisms [25] in the samples, which lead to strong spectral convolution effects. The dispersions of GNPs and GNP/ZnO: Al-NRs grown at $\mathrm{pH} 11$ show a uniform decrease in absorption with excitation time due to particles settling. Figure 3(b) shows that the absorption in GNP/ZnO:Al-NRs grown at a $\mathrm{pH} 6.6$ changes non-uniformly with wavelength. The long wavelength absorption response in our dispersions is dominated by the GNPs, while it relates to $\mathrm{ZnO}$ at short wavelength. The non-uniform decrease in absorption observed in 3(b) indicates the presence of two phases (separated GNPs and $\mathrm{ZnO}: \mathrm{Al}-\mathrm{NRs}$ ) in the dispersion. The $\mathrm{ZnO}: \mathrm{Al}-$ NRs settle faster than the GNPs. In contrast, GNP/ZnO:AlNRs grown at a $\mathrm{pH} 11$ forms a single phase in the dispersion, as evident from the uniform settling rate in figure $3(\mathrm{c})$. This
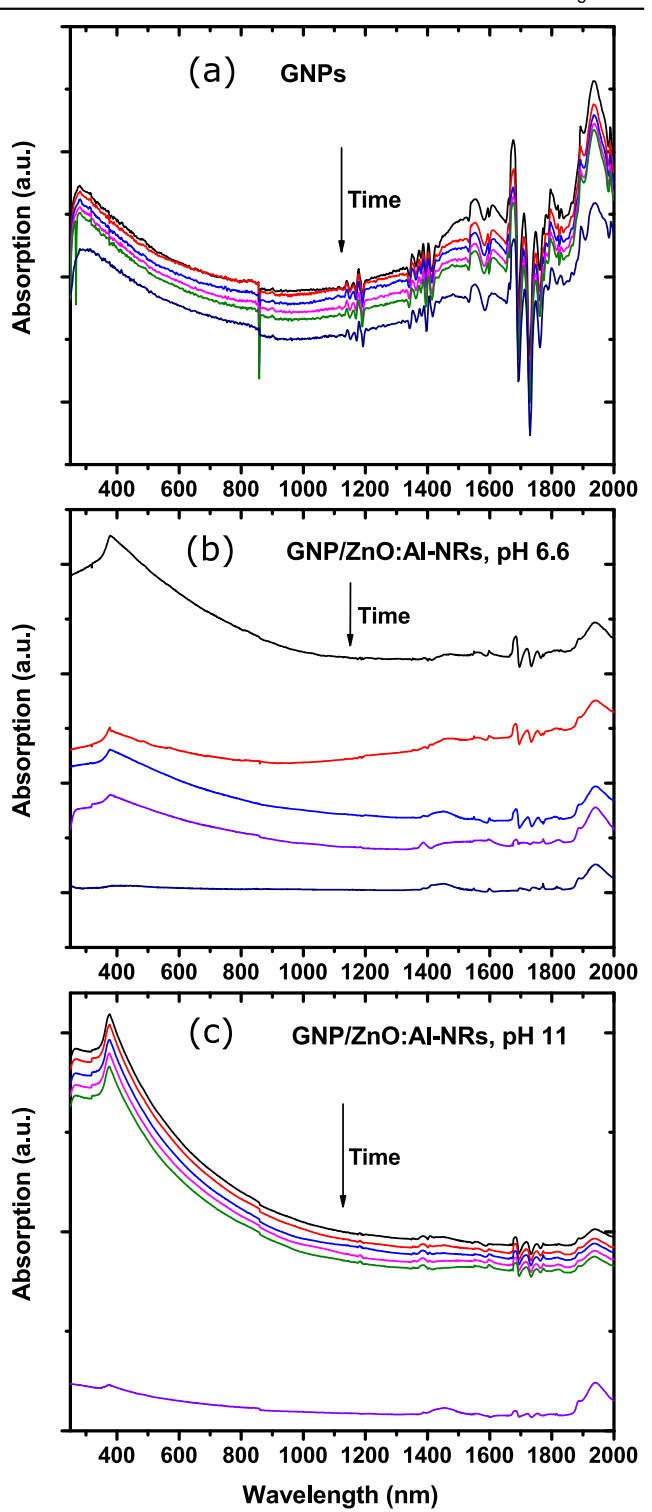

Figure 3. Time-dependent UV-vis spectroscopy. Temporal evolution of the absorption spectra after $0,5,10,15,20 \mathrm{~min}$ and $24 \mathrm{~h}$, respectively, for the dispersion of (a) GNPs, (b) GNP/ZnO:Al-NRs grown at $\mathrm{pH} 6.6$ and (c) GNP/ZnO:Al-NRs grown at $\mathrm{pH} 11$ in isopropanol. (Figure (a) has a different vertical scale.)

implies that $\mathrm{ZnO}$ :Al-NRs are better attached to GNPs in high $\mathrm{pH}$ growth solutions.

To study the quality of bonding between the GNPs and the $\mathrm{ZnO}-\mathrm{NRs}$, and also to see the effects of Al-doping level in 


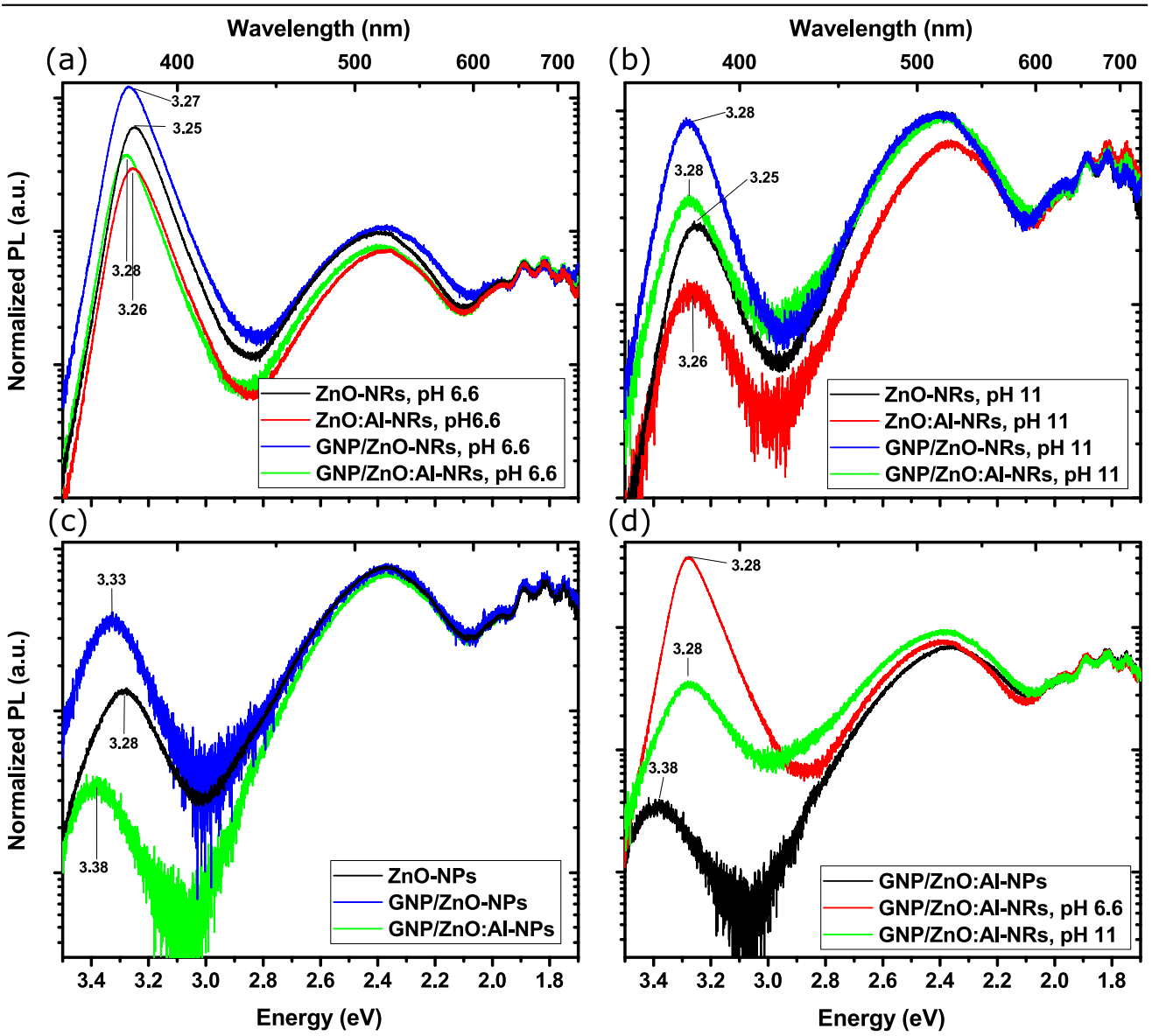

Figure 4. $\mu$-PL room-temperature measurement for different $\mathrm{ZnO}$-NRs composites grown at (a) $\mathrm{pH}$ 6.6, (b) $\mathrm{pH} 11$, and (c) different GNP/ ZnO-NPs composites. (d) Comparing PL spectra for GNP/ZnO:Al composites for three different $\mathrm{ZnO}$ morphologies.

the $\mathrm{ZnO}-\mathrm{NRs}$, room-temperature micro-PL ( $\mu$-PL) measurements were pursued on nanostructure deposits on gold coated silicon wafers using a $80 \mu \mathrm{W}, 266 \mathrm{~nm}$ laser excitation. The main peak in figure 4 shows the $\mathrm{ZnO}$ near-bandedge emission (NBE) at $3.25-3.38 \mathrm{eV}$ in agreement with the absorption measurements. The broad peak at $2.4 \mathrm{eV}$ indicates the visible light emission via deep levels in $\mathrm{ZnO}$ caused by zinc and oxygen vacancies [19]. The origin of the oscillations at $1.7-2 \mathrm{eV}$ are not well understood but they are a common feature for all the samples. For easier comparison, all the PL data were normalized to the peak at $1.9 \mathrm{eV}$. Three sets of $\mathrm{ZnO}$-nanorod morphologies ( $\mathrm{ZnO}-\mathrm{NPs}$ and $\mathrm{ZnO}-\mathrm{NRs}$ grown at $\mathrm{pH} 6.6$ and 11) in four different composites (undoped $\mathrm{ZnO}$, $\mathrm{ZnO}: \mathrm{Al}, \mathrm{GNP} / \mathrm{ZnO}, \mathrm{GNP} / \mathrm{ZnO}: \mathrm{Al}$ ) were investigated under the same conditions (figures 4(a)-(c)).

Two distinct changes are readily observed in the $\mu$-PL spectra after doping the $\mathrm{ZnO}-\mathrm{NRs}$ with $\mathrm{Al}$; first, the deep level emission (DLE) is suppressed due to filling of the defects levels by electrons ( $\mathrm{Al}$ is a donor in $\mathrm{ZnO}$ ). This observation has previously been reported for Ga-doped $\mathrm{ZnO}-\mathrm{NRs}$ [26]. Furthermore, the NBE is also suppressed by Al-doping more significantly. The ratio of NBE-to-DLE integrated intensities $\left(I_{\mathrm{NBE}} / I_{\mathrm{DLE}}\right)$ for different samples are summarized in table 1 . In all the samples the $I_{\mathrm{NBE}} / I_{\mathrm{DLE}}$ ratio is decreased by adding $\mathrm{Al}$ to the composite. This decrease in the UV-to-visible emission ratio can be explained by introducing impurity levels in the bandgap with the doped metal ions and reducing the crystalline quality of $\mathrm{ZnO}$ [27-29]. Although it has been reported that post thermal treatment can improve the $\mathrm{ZnO}: \mathrm{Al}$ crystallinity [28], our samples were measured without thermal treatment. Second, a blue-shift in the band-edge emission is observed which we attribute to the Burstein-Moss effect [23, 27, 30].

Another interesting general observation in the $\mu$-PL spectra is that $\mathrm{ZnO}-\mathrm{NRs}$ grown on GNPs exhibit a significantly stronger 
Table 1. The NBE-to-DLE integrated intensity ratio $\left(I_{\mathrm{NBE}} / I_{\mathrm{DLE}}\right)$ for different ZnO-NRs composites.

\begin{tabular}{lcc}
\hline & Grown at pH 6.6 & Grown at pH 11 \\
\hline ZnO-NRs & 2.46 & 0.15 \\
ZnO:Al-NRs & 1.78 & 0.11 \\
GNP/ZnO-NRs & 4.25 & 0.46 \\
GNP/ZnO:Al-NRs & 1.85 & 0.21 \\
\hline
\end{tabular}

band-edge PL emission intensity compared to isolated $\mathrm{ZnO}$ NRs. Previous reports in the literature attribute this to a reduction in the concentration of oxygen vacancy surface defects in the $\mathrm{ZnO}$ by oxygen groups adsorbed on the GNPs [31,32] or more probably to resonant excitation of graphene plasmons [33-35].

Comparing the $\mu$-PL for the two $\mathrm{ZnO}-\mathrm{NRs}$ morphologies in figure 4(d) shows that thicker $\mathrm{ZnO}-\mathrm{NRs}$ grown at $\mathrm{pH} 6.6$ display a stronger band-edge PL emission than the thinner needle-like $\mathrm{ZnO}-\mathrm{NRs}$ grown at $\mathrm{pH} 11$ due to a lower surfaceto-volume ratio. A high surface defect concentration on the thinner Zn-NRs not only causes a lower band-edge emission in the optical characteristics, but also leads to a higher electrical contact resistance (figure 2(b)).

To further support our conclusions, we measured the spectrally resolved photoconductivity of our samples. A significant persistent photoconductivity (PPC) in $\mathrm{ZnO}$ has been demonstrated in many articles [36-39]. The PPC observed in $\mathrm{ZnO}$ is attributed to a spatial charge separation mechanism induced by the built-in electric field caused by a surface space-charge layer [40]. Oxygen vacancies in the $\mathrm{ZnO}$ structure can chemically absorb oxygen from the air and become a hole trap. During illumination with photons with larger energy than the bandgap of $\mathrm{ZnO}$, photogenerated holes will migrate to these surface traps and photodesorb the oxygen. At this point the separated mobile photoelectrons remain in the $\mathrm{ZnO}$ conduction band and result in a PPC.

Figure 5 below shows the results of pulsed optical excitation of an undoped $\mathrm{GNP} / \mathrm{ZnO}-\mathrm{NR}$ sample at $300 \mathrm{~K}$. The measured current is recorded at a bias of $1 \mathrm{~V}$ under optical excitation with different wavelengths from 320 to $400 \mathrm{~nm}$. There was no detected photoresponse for wavelengths longer than the corresponding bandgap energy. A large PPC effect was observed that increased at shorter wavelengths. In contrast, no PPC was observed in any of the highly Al-doped samples, not even under $254 \mathrm{~nm}$ illumination. This absence of PPC in highly doped samples can be explained by electrostatic screening of the surface oxygen ions that prevents holes from migrating towards the surface [40].

Time-resolved PPC was measured in detail to compare the effect of nanocomposite morphology on the lifetime of the photogenerated electrons. Figure 6 shows the time-dependence PPC of undoped GNP/ZnO-NR samples with different morphology after $350 \mathrm{~nm}$ excitation. After switching on the light, the surface chemisorpted oxygen traps the migrating photogenerated holes leaving behind an increasing density of mobile electrons in the conduction band, which results in an increasing PPC. Eventually, the complex processes involving

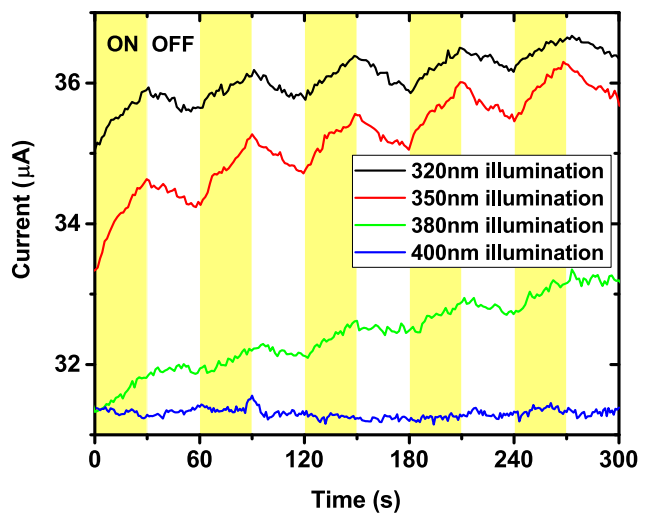

Figure 5. The cycled PPC response for a GNP/ZnO-NRs sample at $300 \mathrm{~K}$ and $1 \mathrm{~V}$ applied bias.

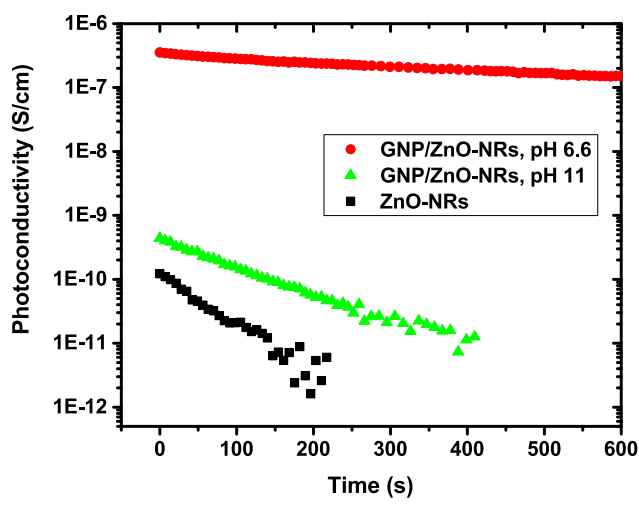

Figure 6. Time-dependence of the PPC $\left(\sigma_{p h}\right)$ after switching off the light for three different GNP/ZnO-NRs morphologies under $350 \mathrm{~nm}$ illumination at $300 \mathrm{~K}$ and $1 \mathrm{~V}$ applied bias.

charge carrier dynamics and surface oxygen chemisorption/ desorption are in balance and the PPC saturates. By switching off the incident light, the PPC slowly decays due to recombination of photogenerated electrons and surface oxygen chemisorption. A detailed analysis of the continuity equation for the investigated complex composite materials is beyond the scope of this paper. Instead we focus on a more qualitative analysis of the decay process.

During the decay, the PPC is proportional to the density of electrons in the conduction band which in general decays stretched exponentially with time [41]. Evidently (figure 6), the photoconductivity (after subtracting the dark conductivity) $\sigma_{p h}$ decays exponentially according to:

$$
\begin{gathered}
\frac{d \sigma_{p h}}{d t}=-\frac{\sigma_{p h}}{\tau_{d}}, \\
\ln \sigma_{p h}=-\frac{t}{\tau_{d}}+\ln \sigma_{0} .
\end{gathered}
$$


Table 2. Dark conductivity $\left(\sigma_{d}\right)$, PPC directly after switching off the light $\left(\sigma_{0}\right)$ and excess electron lifetime $\left(\tau_{d}\right)$ of three different undoped samples at $300 \mathrm{~K}$.

\begin{tabular}{llll}
\hline Sample & $\sigma_{d}\left(\mathrm{~S} \mathrm{~cm}^{-1}\right)$ & $\sigma_{0}\left(\mathrm{~S} \mathrm{~cm}^{-1}\right)$ & $\tau_{d}(\mathrm{~s})$ \\
\hline ZnO-NRs & $1.7 \times 10^{-9}$ & $1.1 \times 10^{-10}$ & 56 \\
GNP/ZnO-NRs, pH 11 & $7.3 \times 10^{-10}$ & $3.9 \times 10^{-10}$ & 107 \\
GNP/ZnO-NRs, pH 6.6 & $2.1 \times 10^{-6}$ & $3 \times 10^{-7}$ & 877 \\
\hline
\end{tabular}

Here, $\tau_{d}$ is the decay time constant (excess electron lifetime) and $\sigma_{0}$ is the observed PPC directly after switching off the light. The extracted electron lifetime for samples with different morphology are calculated by a linear fitting to equation (2) and reported in table 2.

While the ZnO-NRs sample exhibits a long electron lifetime of about $56 \mathrm{~s}$, the composite samples of graphene and $\mathrm{ZnO}-\mathrm{NRs}$ have much longer electron lifetimes (107 and 877 $\mathrm{s})$. The prolonged electron lifetime in these composites can be explained by an efficient transfer of photogenerated electrons to the graphene. This electron injection into the graphene causes a strongly reduced recombination rate and thus an enhanced PPC. The sample grown at $\mathrm{pH} 6.6$ shows a significantly longer electron lifetime compared to the sample grown at $\mathrm{pH} 11$. We conclude that the charge transfer between the $\mathrm{ZnO}-\mathrm{NRs}$ and the graphene is more effective in the samples with thicker NRs grown at lower $\mathrm{pH}$ compared to the thinner needle-like NRs grown at higher $\mathrm{pH}$.

The two morphologies of GNP/ZnO-NRs have different advantages and drawbacks. Thinner ZnO-NRs grown in a high $\mathrm{pH}$ solution exhibit a better dispersity and an improved attachment between the GNPs and ZnO-NRs, but also show a higher resistivity due to more surface defects and lower charge transfer between ZnO-NRs and GNPs. In contrast, the thicker $\mathrm{ZnO}-\mathrm{NRs}$ grown at a lower $\mathrm{pH}$ display a lower resitivity and better charger transfer between $\mathrm{ZnO}-\mathrm{NRs}$ and GNPs, but also a weaker GNP/ZnO-NRs bonding and a poorer dispersity. For conductive printing applications, lower resistance samples with thick $\mathrm{ZnO}$-NRs are preferred. However, if the surface of the nanorod defects could be passivated by a proper post-growth treatment, the thinner GNP/ZnONRs morphology could be advantageous.

Growth and in situ doping of conjugated GNP/ZnO-NRs nanocomposites by a simple solution-based method as demonstrated in this work is economically desirable for mass production. For conductive purposes, the nanocomposite materials can be mixed by different polymers and tested for printing performance. Although we have primarily focused on conductive applications, our reported nanocomposites can be used in any optoelectronic, photochemical or photovoltaic application that requires high-porosity conjugated organic/ semiconductor hybrids with a tunable conductance level.

\section{Conclusion}

In conclusion, we report on hydrothermal solution growth and characterization of different GNP/ZnO-NRs nanocomposites.
The $\mathrm{ZnO}-\mathrm{NRs}$ were degenerately doped by $\mathrm{Al}$ for enhanced conductivity suitable for 3D-printing applications. Two different morphologies of $\mathrm{ZnO}-\mathrm{NRs}$, grown at different $\mathrm{pH}$, were compared with respect to dispersity, GNP/ZnO-NRs bonding quality and conductivity. Our results show that a better graphene dispersion and improved bonding quality between the GNPs and NRs can be achieved in growth solutions with higher $\mathrm{pH}$, however with a lower electrical conductivity in deposited GNP/ZnO-NRs layers. From $\mu$-PL measurements we also infer that surface defects play an increasingly important role for thin $\mathrm{ZnO}-\mathrm{NRs}$ with large surface-to-volume ratio. Although $\mathrm{ZnO}-\mathrm{NRs}$ spacers on the GNPs surface decreases the conductivity between individual GNPs, they provide open free space porosities which promotes its capability to form a composite with other materials like thermoplastics and can be used in conductive bulk printing application, specifically 3D-printing. Finally, we demonstrate a strong enhancement in the PPC in undoped $\mathrm{ZnO}-\mathrm{NRs}$ grown on graphene, compared to bare $\mathrm{ZnO}-\mathrm{NRs}$, which we attribute to an effective transfer of photogenerated electrons from the rods to the graphene.

\section{Acknowledgments}

The authors acknowledge financial support from the Knowledge Foundation, Linköping University and Halmstad University. The authors also thank Emil Nilsson and Urban Bilstrup for fruitful discussions.

\section{ORCID iDs}

Ebrahim Chalangar (it) https://orcid.org/0000-00026850-1552

Omer Nur (ib https://orcid.org/0000-0002-9566-041X

\section{References}

[1] Jakus A E, Secor E B, Rutz A L, Jordan S W, Hersam M C and Shah R N 2015 Three-dimensional printing of high-content graphene scaffolds for electronic and biomedical applications ACS Nano 9 4636-48

[2] Leigh S J, Bradley R J, Purssell C P, Billson D R and D A Hutchins 2012 A simple, low-cost conductive composite material for 3D printing of electronic sensors PLoS One 7 e 49365

[3] Kamyshny A and Magdassi S 2014 Conductive nanomaterials for printed electronics Small 10 3515-35

[4] Bauhofer W and Kovacs J Z 2009 A review and analysis of electrical percolation in carbon nanotube polymer composites Compos. Sci. Technol. 69 1486-98

[5] Kim J H, Lee S, Wajahat M, Jeong H, Chang W S, Jeong H J, Yang J-R, Kim J T and Seol S K 2016 Three-dimensional printing of highly conductive carbon nanotube microarchitectures with fluid ink ACS Nano 10 8879-87

[6] Ramasubramaniam R 2003 Homogeneous carbon nanotube polymer composites for electrical applications Appl. Phys. Lett. 83 2928-30 
[7] Weiss N O, Zhou H, Liao L, Liu Y, Jiang S, Huang Y and Duan X 2012 Graphene: an emerging electronic material Adv. Mater. 24 5782-825

[8] Li D, Muller M B, Gilje S, Kaner R B and Wallace G G 2008 Processable aqueous dispersions of graphene nanosheets Nat. Nanotechnol. 3 101-5

[9] Arapov K, Rubingh E, Abbel R, Laven J, de With G and Friedrich H 2016 Conductive screen printing inks by gelation of graphene dispersions Adv. Funct. Mater. 26 586-93

[10] Gao N and Fang X 2015 Synthesis and development of graphene inorganic semiconductor nanocomposites Chem. Rev. $1158294-343$

[11] Klingshirn C, Fallert J, Zhou H, Sartor J, Thiele C, Maier-Flaig F, Schneider D and Kalt H 201065 years of $\mathrm{ZnO}$ research old and very recent results Phys. Status Solidi b 247 1424-47

[12] Willander M et al 2009 Zinc oxide nanorod based photonic devices: recent progress in growth, light emitting diodes and lasers Nanotechnology 20332001

[13] Amin G, Asif M H, Zainelabdin A, Zaman S, Nur O and Willander M 2011 Influence of $\mathrm{pH}$, precursor concentration, growth time, and temperature on the morphology of $\mathrm{ZnO}$ nanostructures grown by the hydrothermal method J. Nanomater. 2011 5:5

[14] Munshi A M, Dheeraj D L, Fauske V T, Kim D-C, van Helvoort A T J, Fimland B-O and Weman H 2012 Vertically aligned GaAs nanowires on graphite and fewlayer graphene: generic model and epitaxial growth Nano Lett. 12 4570-6

[15] Larson K, Clark A, Appel A, Dai Q, He H and Zygmunt S 2015 Surface-dependence of interfacial binding strength between zinc oxide and graphene RSC Adv. 5 65719-24

[16] Choi W M, Shin K-S, Lee H S, Choi D, Kim K, Shin H-J, Yoon S-M, Choi J-Y and Kim S-W 2011 Selective growth of $\mathrm{ZnO}$ nanorods on $\mathrm{SiO}_{2} / \mathrm{Si}$ substrates using a graphene buffer layer Nano Res. 4 440-7

[17] Lin J, Penchev M, Wang G, Paul R K, Zhong J, Jing X Ozkan M and Ozkan C S 2010 Heterogeneous graphene nanostructures: $\mathrm{ZnO}$ nanostructures grown on large-area graphene layers Small 6 2448-52

[18] Hwang J O, Lee D H, Kim J Y, Han T H, Kim B H, Park M, No $\mathrm{K}$ and Kim S O 2011 Vertical $\mathrm{ZnO}$ nanowires/graphene hybrids for transparent and flexible field emission J. Mater. Chem. $213432-7$

[19] Alnoor H, Pozina G, Khranovskyy V, Liu X, Iandolo D, Willander M and Nur O 2016 Influence of $\mathrm{ZnO}$ seed layer precursor molar ratio on the density of interface defects in low temperature aqueous chemically synthesized $\mathrm{ZnO}$ nanorods/GaN light-emitting diodes J. Appl. Phys. 119 165702

[20] Brsan O A, Hoffmann G G, van der Ven L G J and de With G 2016 Single-walled carbon nanotube networks: the influence of individual tube-tube contacts on the large-scale conductivity of polymer composites Adv. Funct. Mater. 26 4377-85

[21] Fuchs P, Hagendorfer H, Romanyuk Y E and Tiwari A N 2015 Doping strategies for highly conductive Al-doped $\mathrm{ZnO}$ films grown from aqueous solution Phys. Status Solidi a 212 51-5

[22] Miyake M, Fukui H and Hirato T 2012 Preparation of Aldoped $\mathrm{ZnO}$ films by aqueous solution process using a continuous circulation reactor Phys. Status Solidi a 209 945-8

[23] Edinger S, Bansal N, Bauch M, Wibowo R A, Hamid R, Trimmel $\mathrm{G}$ and Dimopoulos T 2017 Comparison of chemical bath-deposited $\mathrm{ZnO}$ films doped with $\mathrm{Al}, \mathrm{Ga}$ and In J. Mater. Sci. 52 9410-23

[24] Chandraiahgari C R, De Bellis G, Balijepalli S K, Kaciulis S, Ballirano P, Migliori A, Morandi V, Caneve L, Sarto F and Sarto M S 2016 Control of the size and density of $\mathrm{ZnO}$ nanorods grown onto graphene nanoplatelets in aqueous suspensions RSC Adv. 6 83217-25

[25] Podbrek P, Drai G, Paramo J A, Strzhemechny Y M, Maek J and Orel Z C 2010 Growth of zinc oxide particles in the presence of silicon CrystEngComm 12 3071-9

[26] Sakurai M, Wang Y G, Uemura T and Aono M 2009 Electrical properties of individual $\mathrm{ZnO}$ nanowires Nanotechnology $\mathbf{2 0}$ 155203

[27] Zhu Q, Xie C, Li H, Yang C, Zhang S and Zeng D 2014 Selectively enhanced UV and NIR photoluminescence from a degenerate $\mathrm{ZnO}$ nanorod array film J. Mater. Chem. C 2 4566-80

[28] Wang M, Lee K E, Hahn S H, Kim E J, Kim S, Chung J S, Shin E W and Park C 2007 Optical and photoluminescent properties of sol-gel Al-doped $\mathrm{ZnO}$ thin films Mater. Lett. 61 1118-21

[29] Rahman M M, Khan M K R, Rafiqul Islam M, Halim M A, Shahjahan M, Hakim M A, Saha D K and Khan J U 2012 Effect of Al doping on structural, electrical, optical and photoluminescence properties of nano-structural $\mathrm{ZnO}$ thin films J. Mater. Sci. Technol. 28 329-35

[30] Ziabari A A and Rozati S M 2012 Carrier transport and bandgap shift in n-type degenerate $\mathrm{ZnO}$ thin films: the effect of band edge nonparabolicity Physica B 407 4512-7

[31] Lee E, Kim J-Y, Kwon B J, Jang E-S and An S J 2014 Vacancy filling effect of graphene on photoluminescence behavior of $\mathrm{ZnO} /$ graphene nanocomposite Phys. Status Solidi 8 836-40

[32] Pham C V, Repp S, Thomann R, Krueger M, Weber S and Erdem E 2016 Charge transfer and surface defect healing within $\mathrm{ZnO}$ nanoparticle decorated graphene hybrid materials Nanoscale 8 9682-7

[33] Hwang S W et al 2010 Plasmon-enhanced ultraviolet photoluminescence from hybrid structures of graphene $/ \mathrm{ZnO}$ films Phys. Rev. Lett. 105127403

[34] Zhang S G, Wen L, Li J L, Gao F L, Zhang X W, Li L H and Li G Q 2014 Plasmon-enhanced ultraviolet photoluminescence from highly ordered $\mathrm{ZnO}$ nanorods/ graphene hybrid structure decorated with $\mathrm{Au}$ nanospheres J. Phys. D: Appl. Phys. 47495103

[35] Liu R, Fu X-W, Meng J, Bie Y-Q, Yu D-P and Liao Z-M 2013 Graphene plasmon enhanced photoluminescence in $\mathrm{ZnO}$ microwires Nanoscale 5 5294-8

[36] Medved D B 1961 Photoconductivity and chemisorption kinetics in sintered zinc oxide semiconductor J. Phys. Chem. Solids 20 255-67

[37] Chen M-W, Chen C-Y, Lien D-H, Ding Y and He J-H 2010 Photoconductive enhancement of single $\mathrm{ZnO}$ nanowire through localized Schottky effects Opt. Express 18 14836-41

[38] Moore J C and C V Thompson 2013 A phenomenological model for the photocurrent transient relaxation observed in ZnO-based photodetector devices Sensors 13 9921-40

[39] Zhu Q, Xie C, Li H, Zhang J and Zeng D 2015 Through-process analytical modeling of photoconductance spectrum for porous $\mathrm{ZnO}$ nanocrystalline film Chem. Mater. 27 2861-74

[40] Zhu Q, Xie C, Li H and Zeng D 2016 A method for modeling and deciphering the persistent photoconductance and long-term charge storage of ZnO nanorod arrays Nano Res. 9 2972-3002

[41] Rani M and Tripathi S K 2016 Electron transfer properties of organic dye sensitized $\mathrm{ZnO}$ and $\mathrm{ZnO} / \mathrm{TiO}_{2}$ photoanode for dye sensitized solar cells Renew. Sustain. Energy Rev. 61 97-107 
PAPER II 


\section{Check for updates}

Cite this: RSC Adv., 2019, 9, 30585

\title{
Graphene-based plasmonic nanocomposites for highly enhanced solar-driven photocatalytic activities
}

\author{
Rania E. Adam, (D) $\dagger^{\star a}$ Ebrahim Chalangar, (D) $\dagger^{\mathrm{ab}}$ Mahsa Pirhashemi, ${ }^{\mathrm{c}}$ Galia Pozina, \\ Xianjie Liu, ${ }^{d}$ Justinas Palisaitis, ${ }^{d}$ Håkan Pettersson, ${ }^{\text {abe }}$ Magnus Willander $^{\mathrm{a}}$ \\ and Omer Nur (D) a \\ High-efficiency photocatalysts are crucial for the removal of organic pollutants and environmental \\ sustainability. In the present work, we report on a new low-temperature hydrothermal chemical method \\ assisted by ultrasonication, to synthesize disruptive plasmonic $\mathrm{ZnO} / \mathrm{graphene/Ag/Agl} \mathrm{nanocomposites}$ \\ for solar-driven photocatalysis. The plasmonic nanocomposites were investigated by a wide range of \\ characterization techniques, confirming successful formation of photocatalysts with excellent \\ degradation efficiency. Using Congo red as a model dye molecule, our experimental results \\ demonstrated a photocatalytic reactivity exceeding $90 \%$ efficiency after one hour simulated solar \\ irradiation. The significantly enhanced degradation efficiency is attributed to improved electronic \\ properties of the nanocomposites by hybridization of the graphene and to the addition of Ag/Agl which \\ generates a strong surface plasmon resonance effect in the metallic silver further improving the \\ photocatalytic activity and stability under solar irradiation. Scavenger experiments suggest that \\ superoxide and hydroxyl radicals are responsible for the photodegradation of Congo red. Our findings \\ are important for the fundamental understanding of the photocatalytic mechanism of $\mathrm{ZnO} / \mathrm{graphene} / \mathrm{Ag} /$ \\ Agl nanocomposites and can lead to further development of novel efficient photocatalyst materials.
}

Received 12th August 2019 Accepted 19th September 2019

DOI: $10.1039 / \mathrm{c} 9 \mathrm{ra06273d}$

rsc.li/rsc-advances explored with the aim to find materials with enhanced photocatalytic properties under solar light irradiation. Zinc oxide ( $\mathrm{ZnO})$ is a remarkable photocatalyst that can be composited with other materials to improve visible light harvesting and, thus, the photocatalytic efficiency. ZnO-graphene nanocomposites are particularly interesting heterostructures with a capability of inhibiting the recombination of photogenerated charge carriers during the photocatalytic process. Graphene (GR) is a well-known 2D material consisting of carbon atoms arranged in a honeycomb lattice structure. ${ }^{11,12}$ It possesses many fundamentally interesting electronic properties such as zero bandgap, zero effective mass, high charge carrier mobility, large surface area, and high optical transparency over a very large spectral range from IR to UV, which makes it an excellent candidate for enhancing the performance of photocatalysts. ${ }^{10,11,13-15}$ It was found that GR and GR oxide can significantly improve the separation efficiency of photogenerated $\mathrm{e}^{-} / \mathrm{h}^{+}$pairs in photocatalytic processes..$^{10,11,16-20}$ For instance, Tian et al. $^{21}$ fabricated $\mathrm{ZnO}$ nanorods/reduced GR oxide nanocomposites for enhancing the photodegradation of methylene blue dye. Also, Sawant et al. ${ }^{22}$ showed an enhancement of the photodegradation capability of methylene orange and rhodamine $\mathrm{B}$ using $\mathrm{ZnO} / \mathrm{GR}$ nanosheets.

Many different metal-semiconductor nanostructures have been studied as plasmonic photocatalysts. ${ }^{6,23,24}$ Recently, Ag- 
based semiconductors exhibiting a strong surface plasmon resonance effect $(\mathrm{SPR})^{23,25-29}$ were extensively investigated as visible light photocatalysts for degradation of organic pollutants and toxic dyes. In order to further increase the photocatalytic activity of the $\mathrm{ZnO} / \mathrm{GR}$ photocatalyst under visible light irradiation, a narrow bandgap silver halide e.g. silver iodide (AgI) can be added to the nanocomposite. ${ }^{25}$ The blending of AgI with $\mathrm{ZnO}$ shifts the absorption of the nanocomposite significantly towards the visible light region. ${ }^{11,27,30}$ In addition, the excellent conductivity of AgI nanostructures can promote electron transfer that suppresses $\mathrm{e}^{-} / \mathrm{h}^{+}$recombination and, thus, enhances the interfacial charge transfer. ${ }^{25}$ Therefore, it is expected that the addition of $\mathrm{Ag} / \mathrm{AgI}$ to $\mathrm{ZnO} / \mathrm{GR}$ would result in a new nanocomposite with excellent photocatalytic performance. So far, to the best of our knowledge, there are no reports on photocatalytic properties of nanocomposites comprising Ag/ AgI and $\mathrm{ZnO} / \mathrm{GR}$ with respect to degradation of CR dye under solar light irradiation.

In the present work, we report on new plasmonic $\mathrm{ZnO} / \mathrm{GR} /$ Ag/AgI nanocomposites with highly enhanced photocatalytic capability under simulated solar light irradiation. The nanocomposites were prepared via an ultrasonic-assisted hydrothermal solution-based procedure and used for photodegradation of CR dyes. First, ZnO/GR nanocomposites were prepared through hydrothermal growth of $\mathrm{ZnO}$ nanoparticles (NPs) on GR nanosheets. ${ }^{20}$ Subsequently, Ag/AgI NPs were grown on the $\mathrm{ZnO} / \mathrm{GR}$ nanocomposites via an ultrasonic irradiation method. ${ }^{20}$ Also, pristine $\mathrm{ZnO}$ NPs and $\mathrm{ZnO} / \mathrm{Ag} / \mathrm{AgI}$ nanocomposites were prepared as reference samples. The $\mathrm{ZnO} / \mathrm{GR} / \mathrm{Ag} / \mathrm{AgI}$ nanocomposite exhibits superior photocatalytic performance compared to $\mathrm{ZnO} \mathrm{NPs}, \mathrm{ZnO} / \mathrm{GR}$, and $\mathrm{ZnO} / \mathrm{Ag} / \mathrm{AgI}$ nanocomposites. The enhanced photodegradation of CR dyes is primarily attributed to increased light absorption and separation of photogenerated charge carriers by the unique heterojunctions formed between the $\mathrm{ZnO}, \mathrm{GR}$, and $\mathrm{Ag} / \mathrm{AgI}$ counterparts. We present a detailed model of the enhanced photocatalytic mechanism based on the calculated band structure profile of the $\mathrm{ZnO} / \mathrm{GR} / \mathrm{Ag} / \mathrm{AgI}$ heterojunctions.

\section{Experimental part}

\subsection{Materials}

All the chemicals used in this work were purchased from Sigma Aldrich and used without any further purification, including the multilayer GR powder, zinc acetate dihydrate $\left(\mathrm{Zn}\left(\mathrm{CH}_{3} \mathrm{COO}\right)_{2}\right.$ $\left.\cdot 2 \mathrm{H}_{2} \mathrm{O}\right)$, potassium hydroxide $(\mathrm{KOH})$, silver nitrate $\left(\mathrm{AgNO}_{3}\right)$, and sodium iodide (NaI). Deionized (DI) water was used in all steps.

\subsection{Samples preparation}

2.2.1 ZnO/GR. The $\mathrm{ZnO} / \mathrm{GR}$ nanocomposite with a GR-toZnO weight ratio of $1: 99$ was synthesized by adding a $10 \mathrm{mg} \mathrm{L}^{-1}$ dispersion of GR powder in a zinc acetate dehydrate solution $(0.01 \mathrm{M})$ in DI water. Subsequently, $\mathrm{KOH}(0.05 \mathrm{M})$ was added dropwise to the above solution at $60{ }^{\circ} \mathrm{C}$ in an ultrasonic bath and kept for 10 minutes. The obtained $\mathrm{ZnO} / \mathrm{GR}$ nanocomposite was then washed in water and acetone and

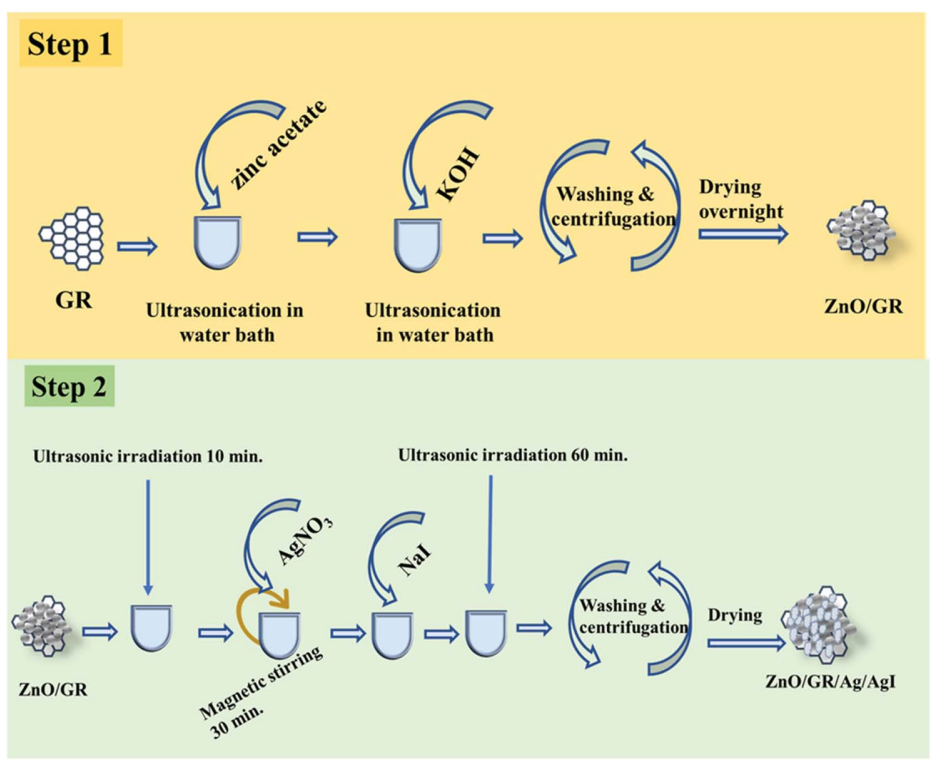

Fig. 1 Schematic diagram for preparation of $\mathrm{ZnO} / \mathrm{GR} / \mathrm{Ag} / \mathrm{Agl}$ nanocomposites. 
centrifuged at $3000 \mathrm{rpm}$ for 10 minutes three times, followed by drying in an oven at $120^{\circ} \mathrm{C}$ overnight.

2.2.2 $\mathrm{ZnO} / \mathbf{G R} / \mathbf{A g} / \mathbf{A g I}$. To prepare the $\mathrm{ZnO} / \mathrm{GR} / \mathrm{Ag} / \mathrm{AgI}$ nanocomposites, $\mathrm{Ag} / \mathrm{AgI}$ was added to the as-prepared $\mathrm{ZnO} / \mathrm{GR}$ with three different weight ratios $X=10 \%, 20 \%$, and $30 \%$ forming the corresponding nanocomposite denoted $\mathrm{ZnO} / \mathrm{GR} / \mathrm{Ag} / \mathrm{AgI}(X \%)$. First, $\mathrm{ZnO} / \mathrm{GR}$ was dispersed into $200 \mathrm{~mL}$ of DI water with ultrasonic irradiation for 10 minutes and then $\mathrm{AgNO}_{3}$ was added to the suspension and stirred for 30 minutes. Next, an aqueous solution of NaI was added dropwise and the final suspension was ultrasonicated for one hour. The nanocomposites were separated by centrifugation and washed two times with DI water and acetone to remove any residuals, and finally dried in an oven at $75{ }^{\circ} \mathrm{C}$ for 6 hours. Fig. 1 shows the preparation steps for fabricating the $\mathrm{ZnO} /$ $\mathrm{GR} / \mathrm{Ag} / \mathrm{AgI}$ nanocomposites.

\subsection{Sample characterization}

Powder X-ray diffraction (XRD) was used to study the structural properties of the prepared samples using a Philips powder diffractometer (1729 PW) equipped with a $\mathrm{Cu} \mathrm{K}(\alpha)$ radiation source at 40 $\mathrm{kV} / 40 \mathrm{~mA}$. The sample morphology was investigated with a fieldemission scanning electron microscope (FE-SEM), equipped with a Sigma 500 Gemini field emission gun operating at $10 \mathrm{kV}$. Samples for transmission electron microscopy (TEM) analysis were prepared by dispersing the obtained nanocomposites onto a lacey carbon film suspended by copper TEM grids. Scanning TEM (STEM) highangle annular dark-field imaging (HAADF), energy-dispersive X-ray spectroscopy (EDS) as well as TEM imaging were performed in the double-corrected Linköping FEI Titan ${ }^{3} 60-300$, operated at 300 $\mathrm{kV}$. The microscope is equipped with image and probe Cs correctors and a monochromated high brightness XFEG gun and an efficient high solid angle Super-X EDX detector. The chemical composition of the samples was investigated by X-ray photoelectron spectroscopy (XPS) using a Scienta ESCA-200 spectrometer equipped with a monochromatic $\mathrm{Al} \mathrm{K}(\alpha)$ X-ray source with a photon energy of $1486.6 \mathrm{eV}$. The optical properties were analyzed by UV-Visible spectrophotometry (PerkinElmer Lambda 900 system) and cathodoluminescence (CL) spectroscopy (Gatan Mono CL4 system combined with FE-SEM). We have also carried out Mott-Schottky tests using a set-up comprising a PGSTAT302N workstation and a standard three-electrode system.

\subsection{Photocatalytic experiments}

The photocatalytic experiments were performed using a solar simulator (100 W ozone-free xenon lamp with a power of $1 \mathrm{sun})$. In brief, each sample $(0.05 \mathrm{~g})$ was mixed with $100 \mathrm{~mL}$ of CR dye solution with an initial concentration of $0.02 \mathrm{~g} \mathrm{~L}^{-1}$ and $\mathrm{pH} \sim 7$ to investigate the photocatalytic activities prior to exposure. These mixtures were then stirred for 30 minutes in dark to reach the adsorption-desorption equilibrium between the photocatalyst and (a)

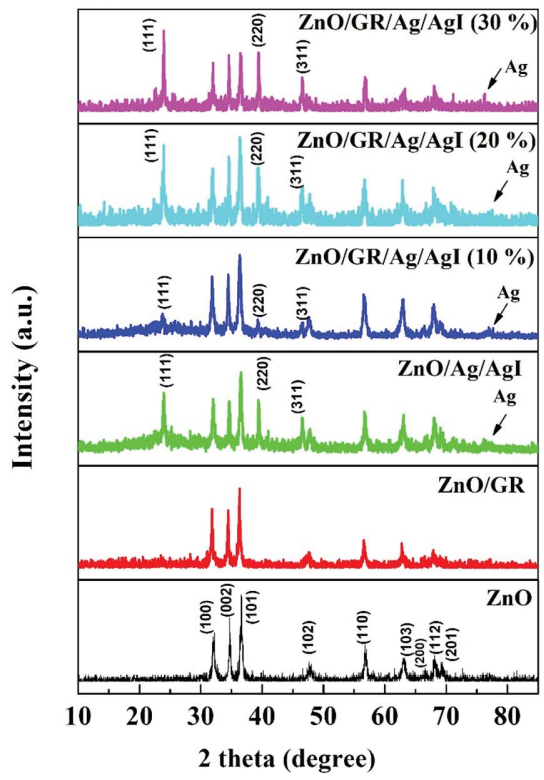

(b)

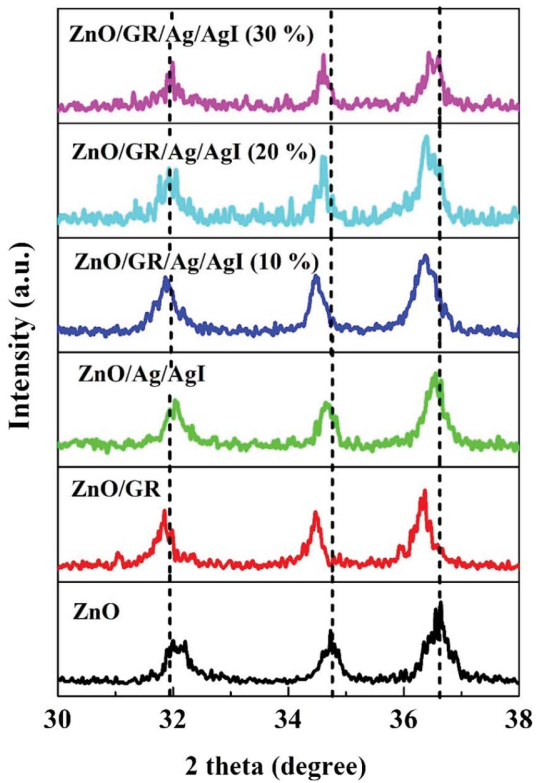

Fig. 2 (a) XRD spectra of pristine ZnO NPs, ZnO/GR, ZnO/Ag/Agl, and ZnO/GR/Ag/Agl nanocomposites with a different weight percentage of $\mathrm{Ag} / \mathrm{Agl}$. (b) High-resolution XRD patterns revealing composition-dependent diffraction peak shifts. 
the dye molecules. After a given interval of irradiation time, a small amount of the mixtures was withdrawn and centrifuged. Finally, the UV-Vis absorbance spectra of the CR dye solution were recorded by the UV-Visible spectrophotometer.

\section{Results and discussion}

\subsection{Analysis of sample characteristics}

Fig. 2 shows the XRD spectra of pristine $\mathrm{ZnO} N P s, \mathrm{ZnO} / \mathrm{GR}, \mathrm{ZnO} /$ $\mathrm{Ag} / \mathrm{AgI}$, and $\mathrm{ZnO} / \mathrm{GR} / \mathrm{Ag} / \mathrm{AgI}$ nanocomposites. It can be observed in Fig. 2(a) that the pristine ZnO NPs exhibit characteristic peaks at $2 \theta$ values of $31.8^{\circ}, 34.6^{\circ}, 36.5^{\circ}, 47.8^{\circ}, 56.8^{\circ}, 63.0^{\circ}, 66.8^{\circ}$, $67.9^{\circ}$, and $69^{\circ}$, which correspond to the crystal planes (100), (002), (101), (102), (110), (103), (200), (112), and (201), respectively, which all belong to the pure hexagonal wurtzite phase of ZnO (JCPDS no. 36-1451). These results strongly imply that the samples are of good crystalline quality and free from defects. Similar XRD diffraction peaks were in fact observed from the $\mathrm{ZnO} / \mathrm{GR}$ nanocomposites. The fact that no diffraction peaks from carbon species were observed can be attributed to the low amount of GR (1 wt\%) in the samples ${ }^{18,19}$ and to the relatively low diffraction intensity of GR. ${ }^{31}$ The presence of GR in the nanocomposites was identified by TEM and XPS analysis (see below). The addition of AgI nanoparticles to the $\mathrm{ZnO}$ and $\mathrm{ZnO} /$ GR nanocomposites show diffraction patterns at $2 \theta$ values of $23.9^{\circ}, 39.2^{\circ}$, and $46.0^{\circ}$, which could be related to the crystal planes (111), (220), and (311), respectively, of the cubic AgI structure (JCPDS no 01-0503). ${ }^{27}$ This conclusion is supported by the observed increase in the intensity of the AgI-related peaks with increasing AgI content. In addition, a small peak at $\sim 77^{\circ}$ was observed in $\mathrm{ZnO} / \mathrm{Ag} / \mathrm{AgI}$ and various $\mathrm{ZnO} / \mathrm{GR} / \mathrm{Ag} / \mathrm{AgI}$ nanocomposites, which can be assigned to the reflections of $\mathrm{Ag}^{0}$ (JCPDS no 65-2871) 27,32 produced during the sample preparation. The effect of GR and Ag/AgI on the crystal structure of the pristine $\mathrm{ZnO}$ NPs was examined by more accurate XRD measurements in the region of $30-40^{\circ}$ as shown in Fig. 2(b). A small shift of the XRD peaks towards smaller angles was not only observed for the $\mathrm{ZnO} / \mathrm{GR}$ nanocomposites compared to the pristine ZnO NPs, but in fact detected for all GR incorporated nanocomposites. This shift indicates that the lattice constants of $\mathrm{ZnO}$ and $\mathrm{Ag} / \mathrm{AgI}$ NPs have changed due to the presence of the carbonaceous material, in agreement with previous reports. ${ }^{33,34}$ The crystallite size of the NPs can be calculated by the Scherrer equation: ${ }^{35}$

$$
D=\frac{k \lambda}{\beta_{h k l} \cos \theta}
$$

Here $D$ is the crystallite size of the ZnO NPs, $\lambda$ is the X-ray wavelength, $\theta$ is the Bragg diffraction angle, $\beta$ is the full width at half maximum of the diffraction peak corresponding to the
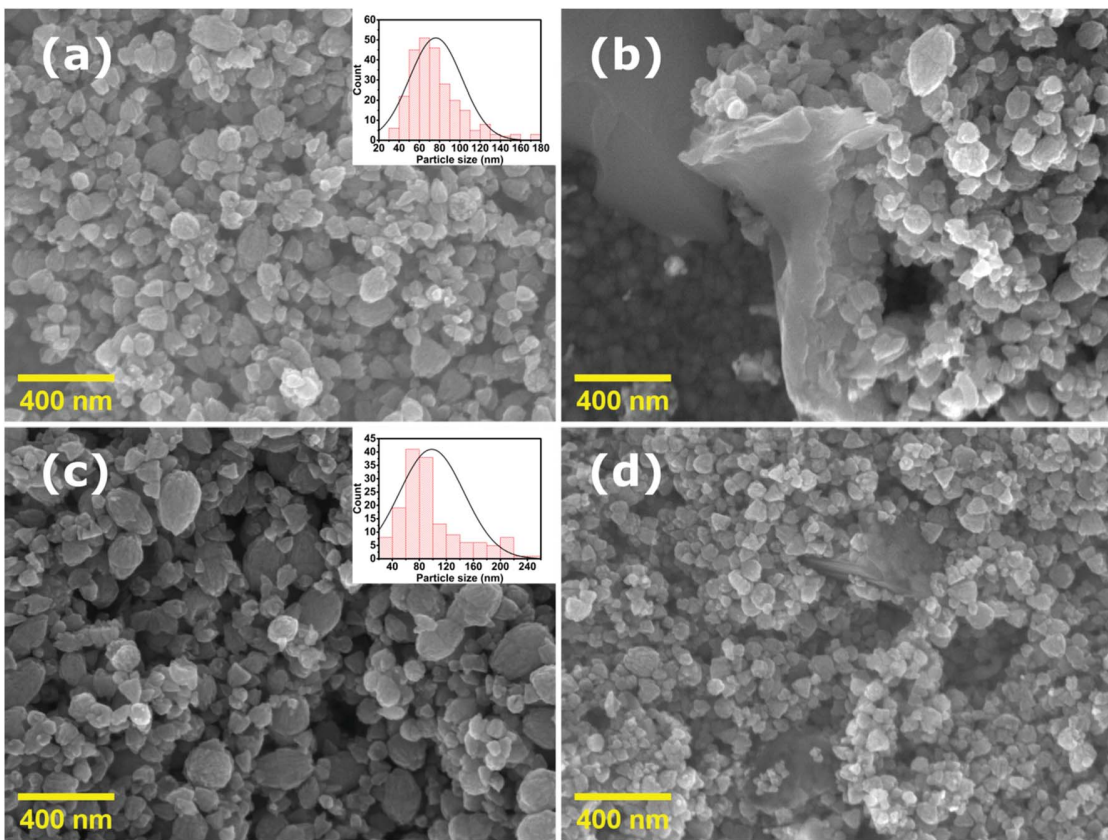

Fig. 3 FE-SEM images of (a) pristine $\mathrm{ZnO} N P s$, (b) $\mathrm{ZnO} / \mathrm{GR}$, (c) $\mathrm{ZnO} / \mathrm{Ag} / \mathrm{Agl}$, and (d) $\mathrm{ZnO} / \mathrm{GR} / \mathrm{Ag} / \mathrm{Agl}$ (20\%) nanocomposites. The insets show the size distribution of the NPS. 


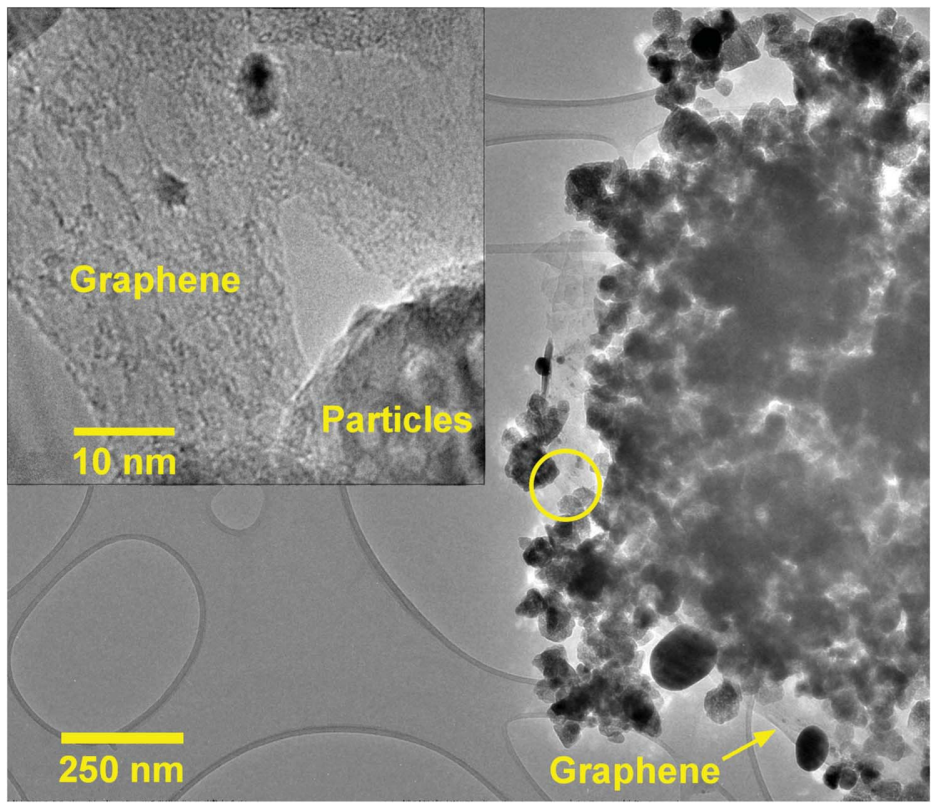

Fig. 4 TEM image of $\mathrm{ZnO} / \mathrm{GR} / \mathrm{Ag} / \mathrm{Agl}(20 \%)$ nanocomposites. The inset shows the conjunction of GR and particles ( $\mathrm{ZnO}$ or $\mathrm{Ag} / \mathrm{Agl})$ at higher magnification (region marked by the yellow circle in the main image).

(101) plane, and $k$ is the Scherrer constant equal to 0.9. It was found that the crystallite size of ZnO NPs is increased slightly after the addition of the $\mathrm{Ag} / \mathrm{AgI}$ from 17 to $20 \mathrm{~nm}$.
Fig. 3 shows FE-SEM images of the synthesized (a) pure $\mathrm{ZnO}$, (b) $\mathrm{ZnO} / \mathrm{GR}$, (c) $\mathrm{ZnO} / \mathrm{Ag} / \mathrm{AgI}$, and (d) $\mathrm{ZnO} / \mathrm{GR} / \mathrm{Ag} / \mathrm{AgI}(20 \%)$ samples. The pure ZnO NPs sample shows a uniform size

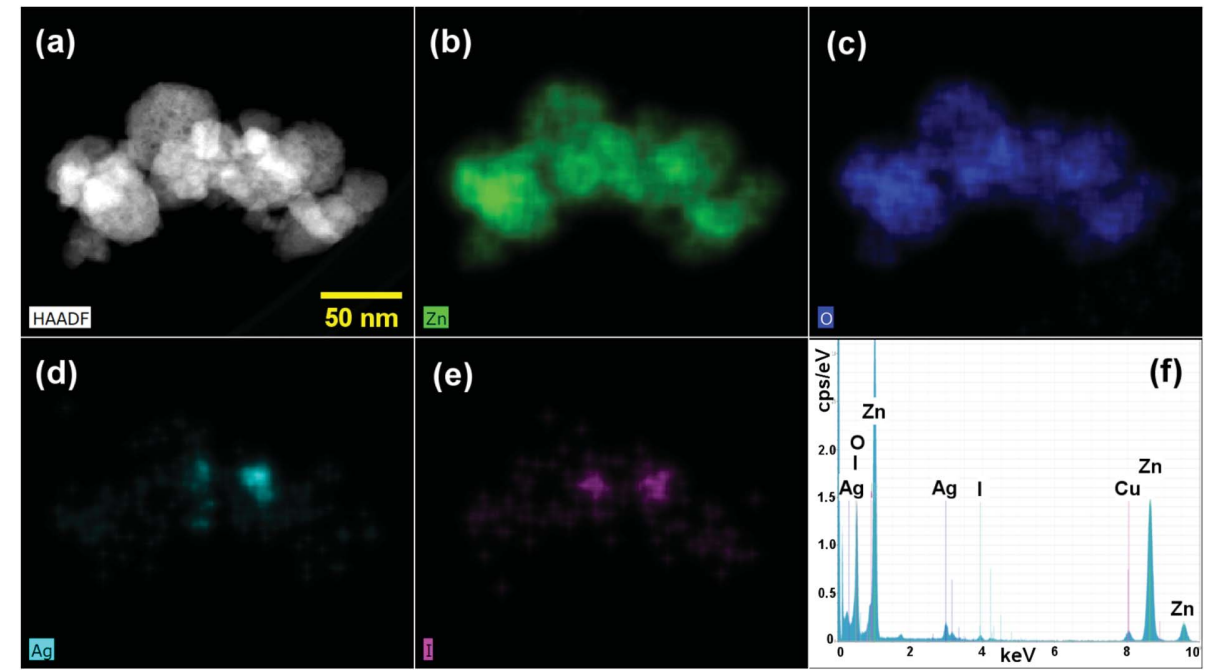

Fig. 5 (a) STEM-HAADF image of a ZnO/GR/Ag/Agl (20\%) nanocomposite, (b-e) STEM-EDS elemental maps displaying Zn, O, Ag, and I distributions, respectively, and (f) integrated EDS spectra. 
distribution as compared to the $\mathrm{ZnO} / \mathrm{Ag} / \mathrm{AgI}$ and $\mathrm{ZnO} / \mathrm{GR} / \mathrm{Ag} / \mathrm{AgI}$ $(20 \%)$ nanocomposites. The average size of the ZnO NPs was found to be $\sim 80 \mathrm{~nm}$, as estimated from the size distribution inset in (a). The average NPs size increased significantly to $\sim 100 \mathrm{~nm}$ (inset in (c)) when $\mathrm{Ag} / \mathrm{AgI}$ was added to the ZnO NPs, which reflects the formation of $\mathrm{ZnO} / \mathrm{Ag} / \mathrm{AgI}$ agglomerates. The particle size observed in the FE-SEM images is much larger than that extracted from the XRD measurements, indicating a polycrystalline structure of the NPs.

TEM investigations confirm the conjunction of NPs and GR nanoplatelets in $\mathrm{ZnO} / \mathrm{GR} / \mathrm{Ag} / \mathrm{AgI}$ (20\%) nanocomposites as shown in Fig. 4. The GR nanoplatelets act as a substructure to assemble the nanoparticles enabling an electrical bridge between them. Additionally, it was observed that the GR nanoplatelets have different size and shape caused by the ultrasonic irradiation during the synthesis procedures.

The elemental distribution of the $\mathrm{ZnO} / \mathrm{GR} / \mathrm{Ag} / \mathrm{AgI}(20 \%)$ nanocomposites were examined using STEM-HAADF imaging (Fig. 5(a)) and STEM-EDS elemental mapping (Fig. 5(b-e)). The integrated EDS spectra, extracted from EDS maps, confirmed the presence of $\mathrm{Zn}, \mathrm{O}, \mathrm{Ag}$, and I elements in the nanocomposites. The corresponding elemental EDS maps revealed that the cluster of NPs forming the nanocomposite is mainly composed of $\mathrm{ZnO}$, while Ag/AgI NPs are present in localized areas of the cluster. Carbon mapping did not display any spectral signatures from GR due to overlap with the underlying carbon support grid (not shown). It is worth noting that a better distribution of the elements in the nanocomposites provides a stronger bond between different counterparts. This leads to a more efficient generation and separation of charge carriers under solar irradiation, which in turn leads to excellent photocatalytic activities and higher photodegradation efficiency.

Furthermore, the chemical oxidation state of the elements in the $\mathrm{ZnO} / \mathrm{GR} / \mathrm{Ag} / \mathrm{AgI}(20 \%)$ nanocomposite was identified by XPS analysis. From the XPS survey scan spectrum in Fig. 6(a) we could clearly identify the existence of $\mathrm{Zn}, \mathrm{O}, \mathrm{C}, \mathrm{Ag}$, and I in the nanocomposite. The high resolution of the $\mathrm{C}$ 1s spectrum shown in Fig. 6(b) was deconvoluted into three Gaussian peaks. Besides the $\mathrm{C}-\mathrm{C}$ bond peak at $284.79 \mathrm{eV}$, which demonstrates the introduced impurities from the surface contamination, ${ }^{36}$ there are two dominant peaks at 285.57 and $288.71 \mathrm{eV}$ assigned to $\mathrm{C}-\mathrm{O}-\mathrm{C}$ and $\mathrm{C}=\mathrm{O}$ bonds, ${ }^{11,36,37}$
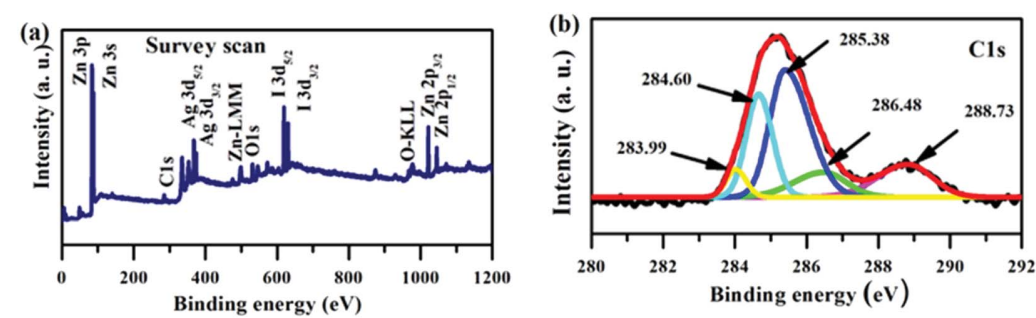

(c)

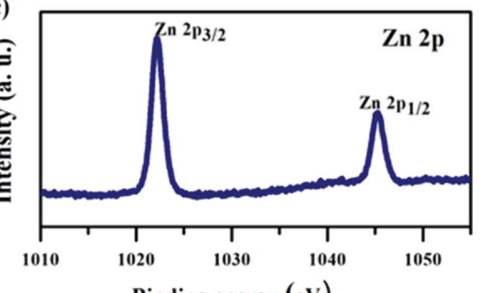

(e)

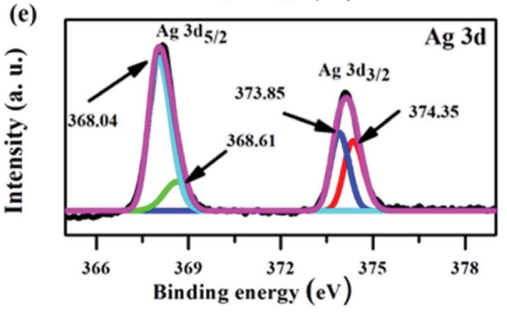

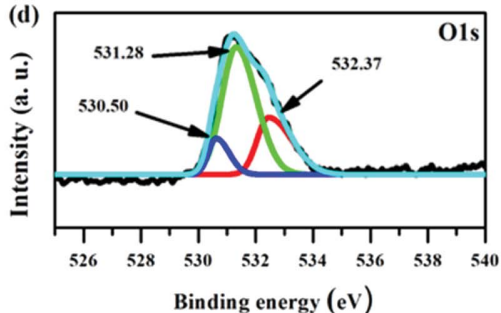

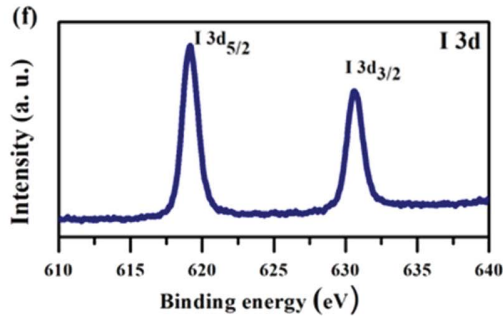

Fig. 6 XPS spectra for a ZnO/GR/Ag/Agl (20\%) nanocomposite. (a) Survey scan, and high resolution core level spectra of (b) C 1s, (c) Zn 2p, (d) O $1 \mathrm{~s}$, (e) Ag 3d, and (f) I 3d. 
respectively. As shown in Fig. 6(c), the XPS core level spectrum of Zn $2 \mathrm{p}$ is composed of two peaks centered at 1022.16 and $1045.23 \mathrm{eV}$, which are ascribed to the binding energy of $\mathrm{Zn} 2 \mathrm{p}_{3 / 2}$ and $\mathrm{Zn} 2 \mathrm{p}_{1 / 2}$ respectively. ${ }^{38}$ The core-level spectrum of $\mathrm{O} 1 \mathrm{~s}$ is presented in Fig. 6(d) and it is divided into two Gaussian fitted peaks. The peak centered at $530.50 \mathrm{eV}$ corresponds to oxygen in the ZnO NPs lattice in the nanocomposite structure..$^{37,39,40}$ The peak at $532.37 \mathrm{eV}$ can be ascribed to oxygen bonded to carbon atoms (the functional group present on GR) and also to the hydroxyl group adhering to the surface of nanocomposite. ${ }^{41,42}$ The XPS spectrum of $\mathrm{Ag} 3 \mathrm{~d}$ is shown in Fig. 6(e). The main peaks at 368.13 and $374.06 \mathrm{eV}$ are assigned to $\mathrm{Ag} 3 \mathrm{~d}_{5 / 2}$ and $\mathrm{Ag} 3 \mathrm{~d}_{3 / 2}$, respectively. The $\mathrm{Ag} 3 \mathrm{~d}_{5 / 2}$ is further divided into two peaks at 367.82 and $368.28 \mathrm{eV}$ and the $\mathrm{Ag} 3 \mathrm{~d}_{3 / 2}$ peak is also divided into two peaks at 373.79 and $374.32 \mathrm{eV}$. The low energy peaks at 367.82 and $373.79 \mathrm{eV}$ are attributed to $\mathrm{Ag}^{+}$, whereas the peaks at 368.28 and $374.32 \mathrm{eV}$ are related to metallic $\mathrm{Ag}^{0}{ }^{0}{ }^{27,43}$ Accordingly, metallic $\mathrm{Ag}^{0}$ is produced at the surface of the $\mathrm{ZnO} / \mathrm{GR} /$ $\mathrm{Ag} / \mathrm{AgI}(20 \%)$ photocatalyst during the sample preparation, an observation consistent with the XRD results shown in Fig. 2(a). The chemical state of the I element, which is present in the form of $\mathrm{I}^{-}$ ions in the nanocomposite, is revealed in Fig. 6(f). Two clearly resolved peaks related to $\mathrm{I} 3 \mathrm{~d}_{5 / 2}$ and $\mathrm{I} 3 \mathrm{~d}_{3 / 2}$ are located at 619.02 and $630.58 \mathrm{eV}$, respectively. ${ }^{30}$

The optical characteristics of the nanocomposites were examined by UV-Vis spectroscopy and CL measurements. The UV-Vis absorbance spectra of $\mathrm{ZnO} \mathrm{NPs}, \mathrm{ZnO} / \mathrm{GR}, \mathrm{ZnO} /$ $\mathrm{Ag} / \mathrm{AgI}$, and $\mathrm{ZnO} / \mathrm{GR} / \mathrm{Ag} / \mathrm{AgI}$ nanocomposites with different weight percent of $\mathrm{Ag} / \mathrm{AgI}$ are shown in Fig. 7(a). It is clear that pure ZnO NPs exhibit a strong interband absorption edge around $400 \mathrm{~nm}$. The weak absorbance in the visible region is attributed to the presence of native point defects. ${ }^{29}$ The addition of GR to ZnO slightly redshifts the absorbance edge. This redshift can be explained by the influence of GR on the surface electric charge in the $\mathrm{ZnO} / \mathrm{GR}$ photocatalyst. ${ }^{10,44}$ In the case of $\mathrm{ZnO} / \mathrm{GR} / \mathrm{Ag} / \mathrm{AgI}$ nanocomposites, a broader absorbance is observed in the visible region $(\lambda>$ $400 \mathrm{~nm}$ ) which increases with the weight percent of $\mathrm{Ag} / \mathrm{AgI}$. Therefore, the blending of $\mathrm{Ag} / \mathrm{AgI}$ into the nanocomposite can increase its photocatalytic performance under solar irradiation. The observed absorbance peak at $\lambda=430 \mathrm{~nm}$ is assigned to the surface plasmon absorption of the $\mathrm{Ag} / \mathrm{AgI}$ NPs, indicating the presence of $\mathrm{Ag}^{0}$ at the surface of the nanocomposites. ${ }^{45}$ As a result, due to contributions from different counterparts, the fabricated plasmonic nanocomposite displays the highest optical absorbance, which is beneficial for the photocatalytic performance under solar irradiation. Fig. 7(b) shows CL spectra of $\mathrm{ZnO}, \mathrm{ZnO} / \mathrm{GR}$, $\mathrm{ZnO} / \mathrm{Ag} / \mathrm{AgI}$, and $\mathrm{ZnO} / \mathrm{GR} / \mathrm{Ag} / \mathrm{AgI}(20 \%)$ samples, measured at room temperature. Interestingly, all samples exhibit a broad emission band covering the whole visible region with a luminescence peak centered at $\sim 600 \mathrm{~nm}$. This band can be attributed to radiative transitions associated with intrinsic deep levels such as oxygen vacancies and interstitials $\left(\mathrm{V}_{\mathrm{O}}, \mathrm{O}_{\mathrm{i}}\right)$, as well as to the presence of hydroxyl groups at the surface of the ZnO NPs. ${ }^{46-48}$ In addition, the observed peak at $\lambda=430 \mathrm{~nm}$ is attributed to surface plasmonenhanced luminescence, induced by the Ag/AgI NPs, in agreement with the UV-Vis spectrum in Fig. 7(a). The $\mathrm{ZnO} /$ GR/Ag/AgI (20\%) nanocomposite exhibits broader band luminescence than that of the pure $\mathrm{ZnO}$ NPs and $\mathrm{ZnO} / \mathrm{GR}$, again consistent with the results presented in Fig. 7(a). Evidently, the presence of $\mathrm{Ag} / \mathrm{AgI}$ has a significant positive effect on the emission properties in the visible region, which in turn is expected to improve the photocatalytic capability of the nanocomposite.

\subsection{Photocatalytic performance}

3.2.1 Photodegradation of toxic wastewater. We studied the photocatalytic activities of $\mathrm{ZnO}, \mathrm{ZnO} / \mathrm{GR}, \mathrm{ZnO} / \mathrm{Ag} / \mathrm{AgI}$ and $\mathrm{ZnO} / \mathrm{GR} / \mathrm{Ag} / \mathrm{AgI}$ samples through the degradation of $\mathrm{CR}$ dye under simulated solar irradiation. Fig. $8(\mathrm{a}-\mathrm{f})$ shows the recorded UV-Vis absorbance of the CR dye mixed with the nanocomposite in $15 \mathrm{~min}$ steps of up to $60 \mathrm{~min}$ irradiation. First, the nanocomposite/CR dye was kept in dark for $30 \mathrm{~min}$ to reach the adsorption-desorption equilibrium state between the photocatalysts and CR molecules. Then the light was turned on, and the absorbance spectra were recorded after each $15 \mathrm{~min}$ step for all samples. It is well-known that carbon-based materials have
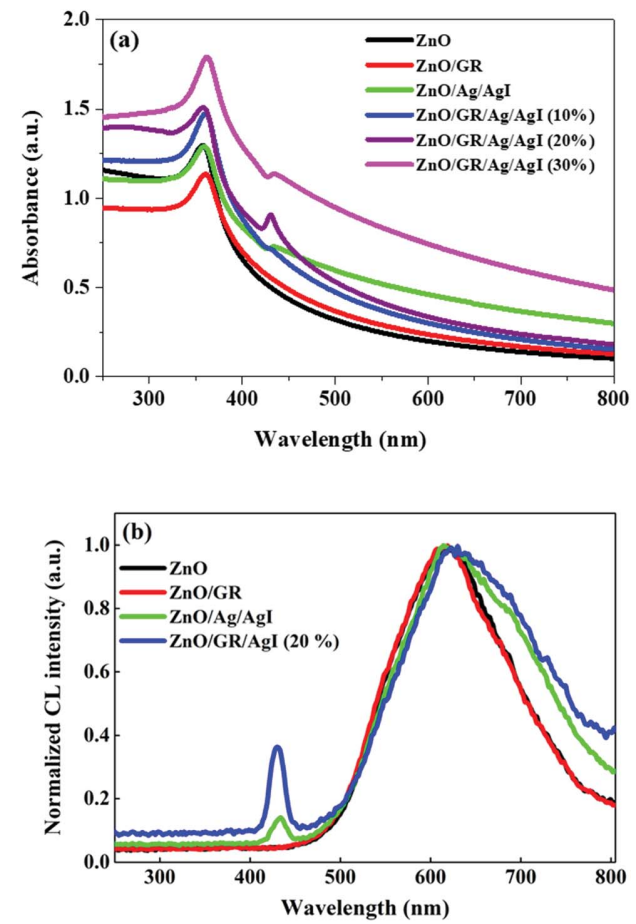

Fig. 7 (a) UV-Vis absorbance spectra of pristine $\mathrm{ZnO} N \mathrm{NP}, \mathrm{ZnO} / \mathrm{GR}$, $\mathrm{ZnO} / \mathrm{Ag} / \mathrm{Agl}$, and $\mathrm{ZnO} / \mathrm{GR} / \mathrm{Ag} / \mathrm{Agl}$ nanocomposites with a different weight percentage of $\mathrm{Ag} / \mathrm{Agl}$, and (b) corresponding $\mathrm{CL}$ spectra. 

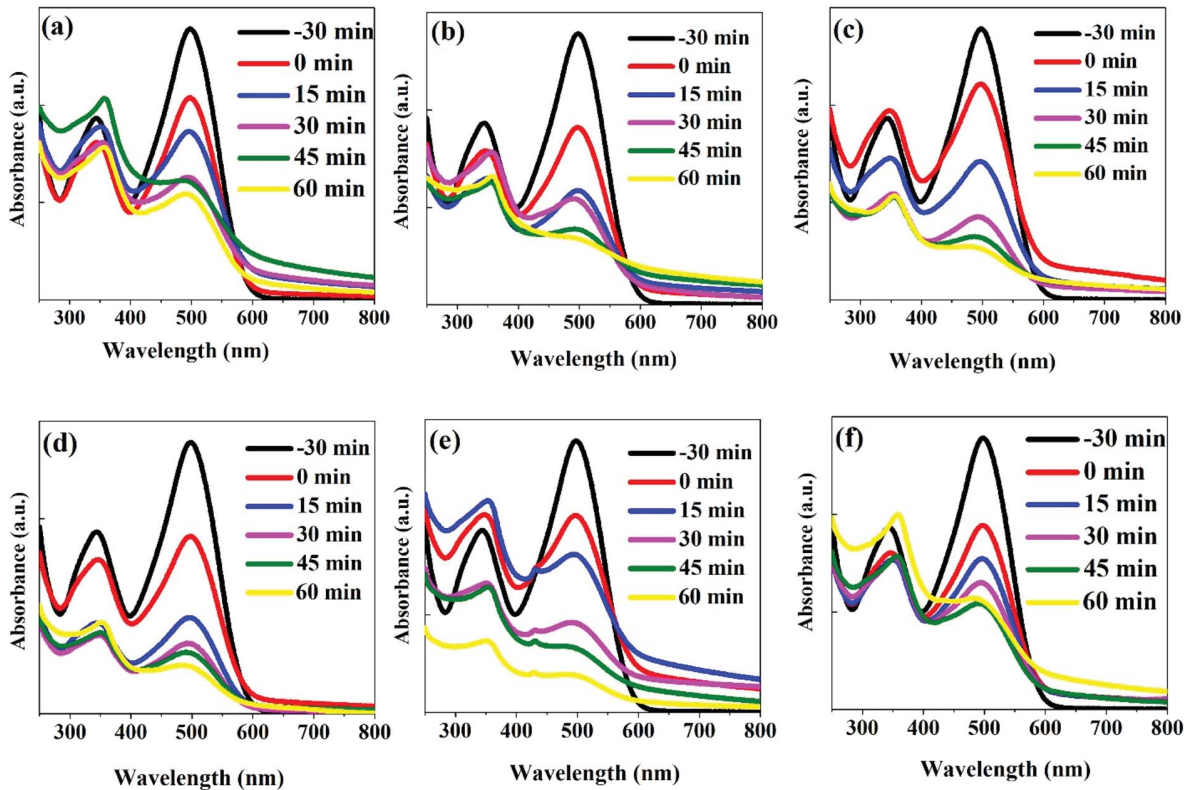

Fig. 8 UV-Vis absorbance spectra of CR dyes recorded after different photodegradation time intervals using (a) ZnO, (b) ZnO/GR, (c) ZnO/Ag/ Agl, (d) ZnO/GR/Ag/Agl (10\%), (e) ZnO/GR/Ag/Agl (20\%), and (f) ZnO/GR/Ag/Agl (30\%) photocatalysts. The spectra marked by "-30 min" were recorded for the $\mathrm{CR}$ only. The spectra marked by "0 $\mathrm{min}$ " were recorded for the samples kept in darkness for 30 min (no irradiation).

an excellent adsorption capacity. This plays a key role in the present nanocomposites where the enhanced dye adsorption on the surface of the photocatalysts strongly increases their photocatalytic performance. ${ }^{49}$ With increasing irradiation time, the main absorbance peak at $497 \mathrm{~nm}$ related to $\mathrm{CR}$ reduces progressively, verifying the degradation of the CR dye. After $60 \mathrm{~min}$ irradiation, the absorbance peak for the $\mathrm{ZnO} / \mathrm{GR} / \mathrm{Ag} / \mathrm{AgI}$ (20\%) nanocomposite has almost disappeared (Fig. 8(e)). In contrast, the variation of the absorbance peaks for the $\mathrm{ZnO}$, $\mathrm{ZnO} / \mathrm{GR}$, and $\mathrm{ZnO} / \mathrm{Ag} / \mathrm{AgI}$ samples were small as shown in Fig. 8(a, b, and c), respectively. The enhanced photocatalytic performance of $\mathrm{ZnO} / \mathrm{GR} / \mathrm{Ag} / \mathrm{AgI}$ nanocomposites can be assigned to the synergistic effect of $\mathrm{ZnO} / \mathrm{GR}$ and $\mathrm{Ag} / \mathrm{AgI}$, and the improved charge transfer ability of the photo-generated carriers at their interfaces as discussed in detail below.

The photodegradation efficiency (\%) was calculated according to eqn (1) and the results are shown in Fig. 9.

$$
\text { Degradation }(\%)=\frac{A_{0}-A}{A_{0}} \times 100
$$

where $A_{0}$ is the initial absorbance of the dye, and $A$ is the absorbance after a specific irradiation time.

Fig. 9(a) displays the calculated degradation efficiency of the $\mathrm{ZnO}, \mathrm{ZnO} / \mathrm{GR}, \mathrm{ZnO} / \mathrm{Ag} / \mathrm{AgI}$, and $\mathrm{ZnO} / \mathrm{GR} / \mathrm{Ag} / \mathrm{AgI}$ samples under solar irradiation. In the absence of any photocatalyst, the photolysis of the $\mathrm{CR}$ is negligible, which shows that this pollutant has high stability during extended periods of irradiation. In contrast, the usage of $\mathrm{ZnO}$ NPs and $\mathrm{ZnO} / \mathrm{GR}$ nanocomposites enhance the photocatalytic performance. In particular, the incorporation of GR leads to a significantly higher photodegradation efficiency of $75 \%$ compared to $58 \%$ for pure $\mathrm{ZnO}$ NPs, an effect primarily attributed to inhibition of the recombination of photogenerated $\mathrm{e}^{-} / \mathrm{h}^{+}$pairs during the photocatalytic reaction. However, in comparison to pure $\mathrm{ZnO}$ NPs, and $\mathrm{ZnO} / \mathrm{GR}$, the plasmonic $\mathrm{ZnO} / \mathrm{Ag} / \mathrm{AgI}$ and $\mathrm{ZnO} / \mathrm{GR} / \mathrm{Ag} /$ AgI nanocomposites show superior photocatalytic activities for CR photodegradation. This important result originates first of all from the addition of $\mathrm{Ag} / \mathrm{AgI}$ which increases the absorption of photons, and thus, the number of photogenerated $\mathrm{e}^{-} / \mathrm{h}^{+}$ pairs in the visible region of the solar spectrum. Interestingly, the photocatalytic performance of the plasmonic nanocomposites depends strongly on the Ag/AgI weight ratio. The $\mathrm{ZnO} / \mathrm{GR} / \mathrm{Ag} / \mathrm{AgI}$ (20\%) nanocomposite exhibits the highest photodegradation efficiency reaching $90 \%$ after $60 \mathrm{~min}$ of irradiation. However, with increasing $\mathrm{Ag} / \mathrm{AgI}$ weight ratio up to $30 \%$, the photodegradation efficiency decreased to only $60 \%$ after 60 minutes of irradiation. This drop in efficiency can be explained by the excess of $\mathrm{Ag} / \mathrm{AgI}$ acting as efficient recombination centers. Moreover, the $\mathrm{Ag} / \mathrm{AgI}$ species might cover the active sites on the nanocomposite surface and thereby passivate 
the effective junctions between the counterparts which lead to a reduced charge separation efficiency in $\mathrm{ZnO} / \mathrm{GR} / \mathrm{Ag} / \mathrm{AgI}(30 \%)$ sample. A specific content of $\mathrm{Ag} / \mathrm{AgI}$ is thus crucial for optimizing the photocatalytic performance of plasmonic $\mathrm{ZnO} / \mathrm{GR} /$ Ag/AgI nanocomposites.

The photodegradation of the CR dye may follow two different processes of direct mineralization or stepwise $\mathrm{N}$-deethylation. The direct mineralization involves gaseous end-products such as $\mathrm{CO}_{2}, \mathrm{NO}_{3}$, and $\mathrm{NO}_{x}$, whereas the stepwise $N$-deethylation leads to a destruction of the conjugated structure of the CR dye which results in a complete degradation. In our photodegradation experiments, the absorption of the $\mathrm{CR}$ is decreasing with time without any shift in the absorbance spectrum after $60 \mathrm{~min}$ of irradiation (Fig. 8(e)). This suggests that the $\mathrm{CR}$ dye was directly mineralized, producing endproducts of $\mathrm{CO}_{2}, \mathrm{NO}_{3}$, and $\mathrm{NO}_{x}{ }^{15,50,51}$

To further reveal the photoreactivity mechanisms involved in photodegradation of the CR dye with the $\mathrm{ZnO} / \mathrm{GR} / \mathrm{Ag} / \mathrm{AgI}(20 \%)$ nanocomposite, trapping experiments were performed to identify the reactive species that are involved in the photocatalytic mechanisms. For this purpose, ethylenediaminetetraacetic acid (EDTA), benzoquinone (BQ) and 2-propanol were used as scavengers for $\mathrm{h}^{+}$, superoxide radicals, and hydroxyl radicals, respectively. ${ }^{52-55}$ As shown in Fig. 9(b), the photodegradation process was suppressed with the addition of $\mathrm{BQ}$ due to the quenching of the superoxide radicals, indicating that the superoxide radicals are the main reactive species involved in the photodegradation of the CR dye. Also, it was observed that the addition of 2-propanol showed partial inhabitation of the degradation of the CR, suggesting that the hydroxyl radicals also play an important role for the photocatalytic performance. Surprisingly, the addition of EDTA did not have any noticeable effect at the beginning of the degradation process. However, the quenching of holes due to the addition of the EDTA gradually increased after $30 \mathrm{~min}$ of irradiation.

To further investigate the photocatalytic stability and reusability of the $\mathrm{ZnO} / \mathrm{GR} / \mathrm{Ag} / \mathrm{AgI}(20 \%)$ nanocomposite, recycling tests of the efficiency for photodegradation of CR were performed. After four successive degradation experiments (Fig. 9(c)), only a small reduction in the efficiency was observed, indicating an excellent stability of the nanocomposite.

To rule out any effects of photosensitization of the CR dye under visible light, the photocatalytic performance of the various nanocomposites in this work was investigated for colourless phenol $\left(0.02 \mathrm{~g} \mathrm{~L}^{-1}\right)$, well known to be insensitive to visible light. A mixture of $100 \mathrm{~mL}$ of a phenol solution with
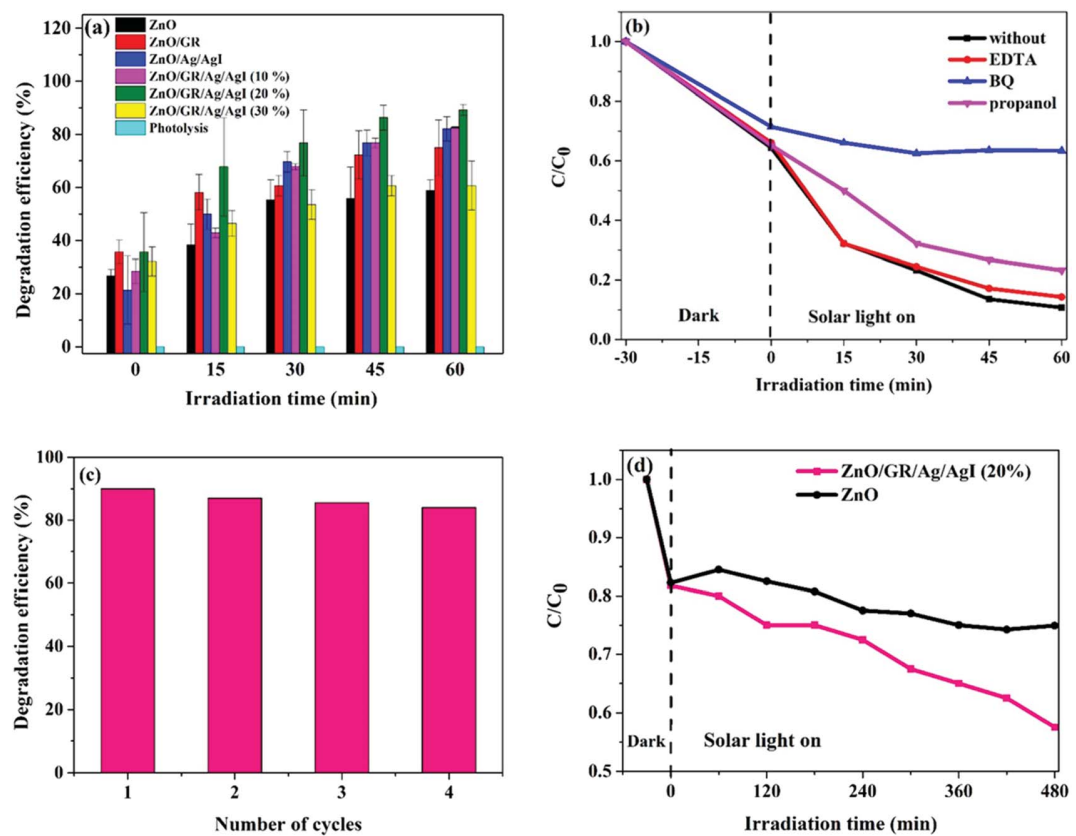

Fig. 9 (a) Photodegradation efficiency of $\mathrm{CR}$ dyes using $\mathrm{ZnO}, \mathrm{ZnO} / \mathrm{GR}, \mathrm{ZnO} / \mathrm{Ag} / \mathrm{Agl}$ and $\mathrm{ZnO} / \mathrm{GR} / \mathrm{Ag} / \mathrm{Agl}$ photocatalysts with different weight percentage of $\mathrm{Ag} / \mathrm{Agl}$, (b) photocatalytic activities of CR with $\mathrm{ZnO} / \mathrm{GR} / \mathrm{Ag} / \mathrm{Agl}(20 \%)$ nanocomposite in the presence of different scavengers, (c) recycling of $\mathrm{ZnO} / \mathrm{GR} / \mathrm{Ag} / \mathrm{Agl}(20 \%)$ nanocomposite during degradation of $\mathrm{CR}$, and (d) photodegradation of phenol with $\mathrm{ZnO}$ and $\mathrm{ZnO} / \mathrm{GR} / \mathrm{Ag} / \mathrm{Agl}$ (20\%) nanocomposite. 
$0.05 \mathrm{~g}$ of the respective nanocomposite was stirred for $30 \mathrm{~min}$ in dark to reach the adsorption-desorption equilibrium. Then UVVis absorbance spectra of the different mixtures were recorded in $60 \mathrm{~min}$ time intervals. The absorption peak of phenol at $270 \mathrm{~nm}$ was used to calculate the concentration of phenol at each time (Fig. 9(d)). It is obvious that the concentration of phenol is decreasing gradually with irradiation time, indicating the decomposition of phenol. In comparison to pure ZnO NPs, the $\mathrm{ZnO} / \mathrm{GR} / \mathrm{Ag} / \mathrm{AgI}(20 \%)$ nanocomposite shows a superior efficiency for photodegradation of phenol. This result also implies that the effect of dye sensitization on the degradation efficiency is small for both phenol and CR.

To develop a deeper understanding of the photocatalytic mechanisms, the apparent reaction constant for each photocatalyst was investigated according to the Langmuir-Hinshelwood's pseudo-first order kinetics model ${ }^{56}$ shown in eqn (2) below.

$$
K_{\text {app }}=\frac{\ln \left[\frac{C_{0}}{C(t)}\right]}{t},
$$

where $K_{\text {app }}$ is the apparent reaction constant $\left(\mathrm{min}^{-1}\right), t$ is the irradiation time (min), $C_{0}$ is the initial concentration $\mathrm{mol} \mathrm{L}^{-1}$ of the dye, and $C(t)$ is the concentration $\mathrm{mol} \mathrm{L}^{-1}$ of the dye after irradiation time $t$. The plot of $\ln \left[\frac{C_{0}}{C(t)}\right]$ versus irradiation time is shown in Fig. 10(a) and found to be in excellent agreement with the Langmuir-Hinshelwood model. The degradation rate constant of CR is found to be $105 \times 10^{-4}, 160 \times 10^{-4}, 208 \times$ $10^{-4}, 208 \times 10^{-4}, 266 \times 10^{-4}$, and $110 \times 10^{-4} \mathrm{~min}^{-1}$ for $\mathrm{ZnO}$, $\mathrm{ZnO} / \mathrm{GR}, \mathrm{ZnO} / \mathrm{Ag} / \mathrm{AgI}, \mathrm{ZnO} / \mathrm{GR} / \mathrm{Ag} / \mathrm{AgI}$ (10\%), $\mathrm{ZnO} / \mathrm{GR} / \mathrm{Ag} / \mathrm{AgI}$ (20\%), and $\mathrm{ZnO} / \mathrm{GR} / \mathrm{Ag} / \mathrm{AgI}(30 \%)$, respectively. It is clear that the degradation rate constant for $\mathrm{ZnO} / \mathrm{GR} / \mathrm{Ag} / \mathrm{AgI}(20 \%)$ is superior to those of the other GR-based nanocomposites.

In Table 1 we show a comparison of the degradation efficiency of different reported GR-based photocatalysts. Summarized, this survey demonstrates that the present plasmonic $\mathrm{ZnO} /$ GR/Ag/AgI (20\%) nanocomposite has very high photodegradation efficiency with respect to the irradiation time of the photocatalytic reaction and with reasonable degradation rate constant.

3.2.2 Proposed photocatalytic mechanism. To experimentally probe the electronic band structure of $\mathrm{ZnO} / \mathrm{Gr} / \mathrm{Ag} / \mathrm{AgI}$ nanocomposites, Mott-Schottky analysis of both $\mathrm{ZnO}$ and AgI semiconductors were performed to determine the conduction and valence band edge energies $\left(E_{\mathrm{CB}}\right.$ and $\left.E_{\mathrm{VB}}\right) .^{57,58}$ As readily observed in Fig. 11(a and b), both the $\mathrm{ZnO}$ and AgI samples exhibit capacitance $v s$. potential characteristics consistent with n-type semiconductors. The flat band potentials can be determined from the extrapolation of $1 / C^{2}$ to zero, which found to be $-0.44 \mathrm{~V}$ and $-0.52 \mathrm{~V} v$ s. $\mathrm{Ag} / \mathrm{AgCl}$ (i.e., $-0.24 \mathrm{~V}$ and $-0.32 \mathrm{~V} v s$. $\mathrm{NHE}$ ) for ZnO and AgI semiconductors, respectively. It is worth noted that the flat band potential for n-type semiconductor is placed at $0.1 \mathrm{eV}$ more positive potential than the $E_{\mathrm{CB}}$. Accordingly, $E_{\mathrm{CB}}$ for the $\mathrm{ZnO}$ and $\mathrm{AgI}$ was obtained to be -0.34 and
$-0.42 \mathrm{eV}$, respectively. In addition, considering the band gap values and based on Mullikan theory calculations, ${ }^{59}$ the $E_{\mathrm{VB}}$ of $\mathrm{ZnO}$ and AgI was computed to be $+2.86 \mathrm{eV}$ and $+2.37 \mathrm{eV}$, respectively.

In Fig. 12 we show a schematic of the proposed photocatalytic mechanism deduced from the combined results of the different measurements and analysis methods discussed in this work. In the presence of solar light, $\mathrm{ZnO}$ and AgI simultaneously play a key role in absorbing the solar light. Especially AgI, with its smaller bandgap than ZnO, can efficiently increase the absorption in the visible light region. ${ }^{27}$ When solar light impinges on the $\mathrm{ZnO} / \mathrm{GR} / \mathrm{Ag} / \mathrm{AgI}$ photocatalyst surface, electrons, and holes are generated in the conduction band (CB) and valence band (VB) in $\mathrm{ZnO}$ and AgI, respectively. Owing to the band offsets, the electrons residing in the $\mathrm{CB}$ of $\mathrm{AgI}$ migrate to the $\mathrm{CB}$ of $\mathrm{ZnO}$ and, in a likewise manner, holes in the $\mathrm{VB}$ of $\mathrm{ZnO}$ move to the $\mathrm{VB}$ of AgI. This leads to an efficient charge carrier separation and transportation which mitigates the undesired recombination of charge carriers. In addition, the plasmonic Ag NPs act as efficient generators of electrons and holes when
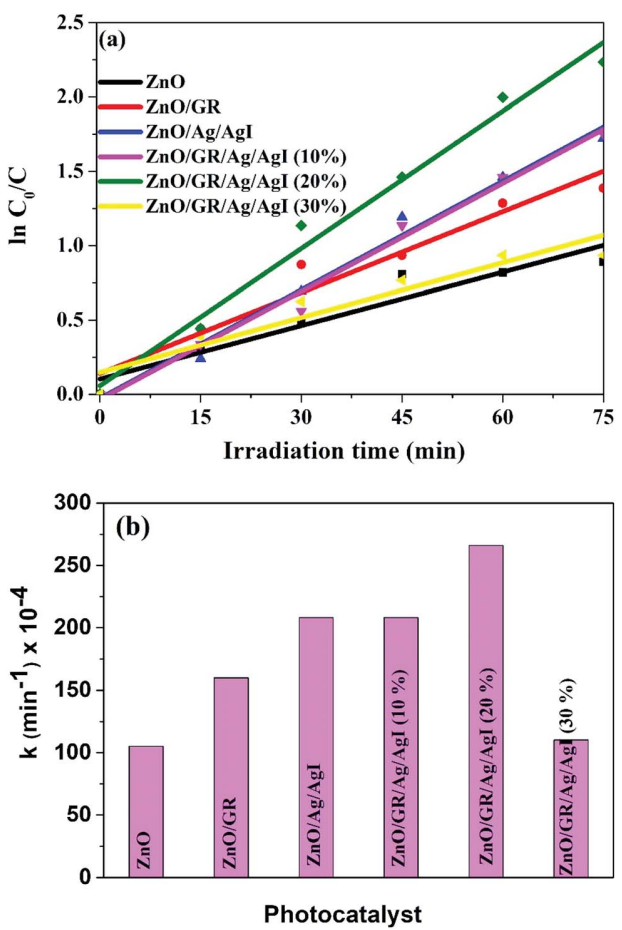

Fig. 10 (a) $\ln \left(C_{0} / C\right)$ versus irradiation time, and (b) degradation rate constant of $\mathrm{CR}$ dyes using $\mathrm{ZnO}, \mathrm{ZnO} / \mathrm{GR}, \mathrm{ZnO} / \mathrm{Ag} / \mathrm{Agl}$ and $\mathrm{ZnO} / \mathrm{GR}$ $\mathrm{Ag} / \mathrm{Agl}$ photocatalysts with a different weight percentage of $\mathrm{Ag} / \mathrm{Agl}$. 
Table 1 Comparison of state-of-the-art GR-based nanocomposites for photodegradation of organic dyes

\begin{tabular}{|c|c|c|c|c|c|c|}
\hline Photocatalyst & Synthesis method & Light source & Dye & $\begin{array}{l}\text { Degradation min/ } \\
\text { efficiency }\end{array}$ & $\begin{array}{l}K_{\text {app }} \\
\left(10^{-2} \min ^{-1}\right)\end{array}$ & Year \\
\hline $\begin{array}{l}\mathrm{AgI}-\mathrm{mesoTiO}_{2} / \\
4 \mathrm{wt} \% \mathrm{RGO}\end{array}$ & Hummers and ultrasound & $150 \mathrm{~W}$ tungsten lamp & $\begin{array}{l}\text { Methylene } \\
\text { orange }\end{array}$ & $90 / 92 \%$ & - & $\begin{array}{l}2016 \text { (ref. } \\
11)\end{array}$ \\
\hline P25/1 wt $\%$ GR & Modified Hummers method & $500 \mathrm{~W}$ xenon lamp & $\begin{array}{l}\text { Methylene } \\
\text { blue }\end{array}$ & $65 / 65 \%$ & - & $\begin{array}{l}\text { 2010 (ref. } \\
60)\end{array}$ \\
\hline $\mathrm{ZnSe} / 1 \mathrm{wt} \% \mathrm{GR}$ & Modified Hummers method & $\begin{array}{l}\text { Solar simulator with a power of } \\
1 \text { sun }\end{array}$ & $\begin{array}{l}\text { Methylene } \\
\text { blue }\end{array}$ & $240 / 99.5 \%$ & 1.27 & $\begin{array}{l}2015 \text { (ref. } \\
61 \text { ) }\end{array}$ \\
\hline $\begin{array}{l}\mathrm{Ag} / \mathrm{Ag}_{2} \mathrm{CO}_{3}-1 \mathrm{wt} \% \\
\mathrm{RGO}\end{array}$ & $\begin{array}{l}\text { Physical mixing and chemical } \\
\text { bath deposition }\end{array}$ & $350 \mathrm{~W}$ xenon lamp & $\begin{array}{l}\text { Methylene } \\
\text { orange }\end{array}$ & $15 / 7 \%$ & 1.80 & $\begin{array}{l}\text { 2016 (ref. } \\
62 \text { ) }\end{array}$ \\
\hline $\begin{array}{l}\mathrm{BiVO}_{4} / 5.5 \mathrm{wt} \% \\
\mathrm{RGO} / \mathrm{Bi}_{2} \mathrm{O}_{3}\end{array}$ & Chemical bath deposition & $150 \mathrm{~W}$ metal halide lamp & Bisphenol A & $240 / 60 \%$ & 0.031 & $\begin{array}{l}\text { 2017 (ref. } \\
63 \text { ) }\end{array}$ \\
\hline $\mathrm{Ag} / \mathrm{ZnO} / 1 \mathrm{wt} \% \mathrm{GR}$ & $\begin{array}{l}\text { Low-temperature microwave- } \\
\text { assisted solution }\end{array}$ & $300 \mathrm{~W}$ high-pressure Hg lamp & $\begin{array}{l}\text { Methylene } \\
\text { orange }\end{array}$ & $80 / 99.6 \%$ & 0.5 & $\begin{array}{l}2015 \text { (ref. } \\
64 \text { ) }\end{array}$ \\
\hline $1.78 \mathrm{wt} \% \mathrm{RGO}-\mathrm{ZnO}$ & Chemical bath deposition & $400 \mathrm{~W} \mathrm{Hg}$ lamp & Rhodamine B & $60 / 61.5 \%$ & - & $\begin{array}{l}2015 \text { (ref. } \\
65 \text { ) }\end{array}$ \\
\hline $3 \mathrm{wt} \% \mathrm{GR} / \mathrm{ZnO}$ & Hydrolysis deposition & 3 W LED lamp & X3B & $45 / 80 \%$ & 0.34 & $\begin{array}{l}2015 \text { (ref. } \\
66 \text { ) }\end{array}$ \\
\hline $\mathrm{ZnO} / 10 \mathrm{wt} \% \mathrm{RGO}$ & Hummers and arc discharge & $90 \mathrm{~W}$ halogen light & $\begin{array}{l}\text { Methylene } \\
\text { orange }\end{array}$ & $90 / 100 \%$ & 0.47 & $\begin{array}{l}2015 \text { (ref. } \\
67)\end{array}$ \\
\hline $1 \mathrm{wt} \% \mathrm{GR} / \mathrm{ZnO}$ & Hydrothermal & $250 \mathrm{~W}$ mercury lamp & $\begin{array}{l}\text { Methylene } \\
\text { blue }\end{array}$ & $450 / 90 \%$ & - & $\begin{array}{l}\text { 2016 (ref. } \\
68 \text { ) }\end{array}$ \\
\hline $\mathrm{Ag}_{2} \mathrm{CrO}_{4}-1 \mathrm{wt} \% \mathrm{GR}$ & Precipitation & $300 \mathrm{~W}$ xenon lamp & $\begin{array}{l}\text { Methylene } \\
\text { blue }\end{array}$ & $15 / 50 \%$ & 2.80 & $\begin{array}{l}2015 \text { (ref. } \\
69)\end{array}$ \\
\hline $\begin{array}{l}\mathrm{ZnO} / 1 \mathrm{wt} \% \mathrm{GR} / \mathrm{Ag} / \\
\mathrm{AgI}\end{array}$ & $\begin{array}{l}\text { Ultrasonic-assisted } \\
\text { hydrothermal }\end{array}$ & $\begin{array}{l}\text { Solar simulator ( } 100 \mathrm{~W} \text { xenon } \\
\text { lamp with a power of } 1 \text { sun) }\end{array}$ & Congo red & $60 / 90 \%$ & 2.66 & $\begin{array}{l}\text { Current } \\
\text { work }\end{array}$ \\
\hline
\end{tabular}
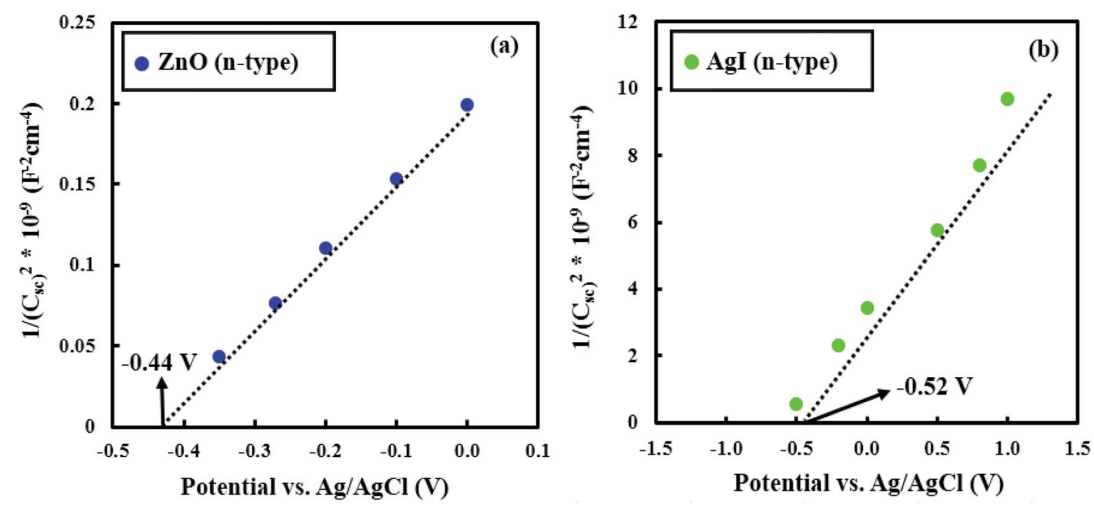

Fig. 11 Mott-Schottky plots for (a) ZnO and (b) Agl samples.

excited by visible light via the SPR effect. These generated electrons are subsequently injected into the $\mathrm{CB}$ of AgI and $\mathrm{ZnO}$, respectively. ${ }^{15,37}$ Subsequently, the photogenerated electrons are transferred from the $\mathrm{CB}$ of $\mathrm{ZnO}$ to the GR. The GR plays a dual role of electron acceptor and transporter, which improve the photocatalytic behavior of the nanocomposite under solar irradiation. The efficient transfer of electrons and the reduction of the recombination rate at the surface of the photocatalyst strongly enhance the photocatalytic efficiency. The trapped electrons react with oxygen molecules to produce superoxide radicals that subsequently get converted into hydroxyl radicals through multi-electron reduction reactions. Finally, these active species can react with pollutant (dye) molecules and decompose them into $\mathrm{H}_{2} \mathrm{O}, \mathrm{CO}_{2}$, and mineral compounds. The photoinduced holes can directly oxidize the adsorbed dye molecules to produce degraded products. As a result, the high photodegradation efficiency of $\mathrm{ZnO} / \mathrm{GR} / \mathrm{Ag} / \mathrm{AgI}$ nanocomposites is related to the uniform distribution of the nanoparticles at the surface of the GR nanosheets, and strong synergy effects 


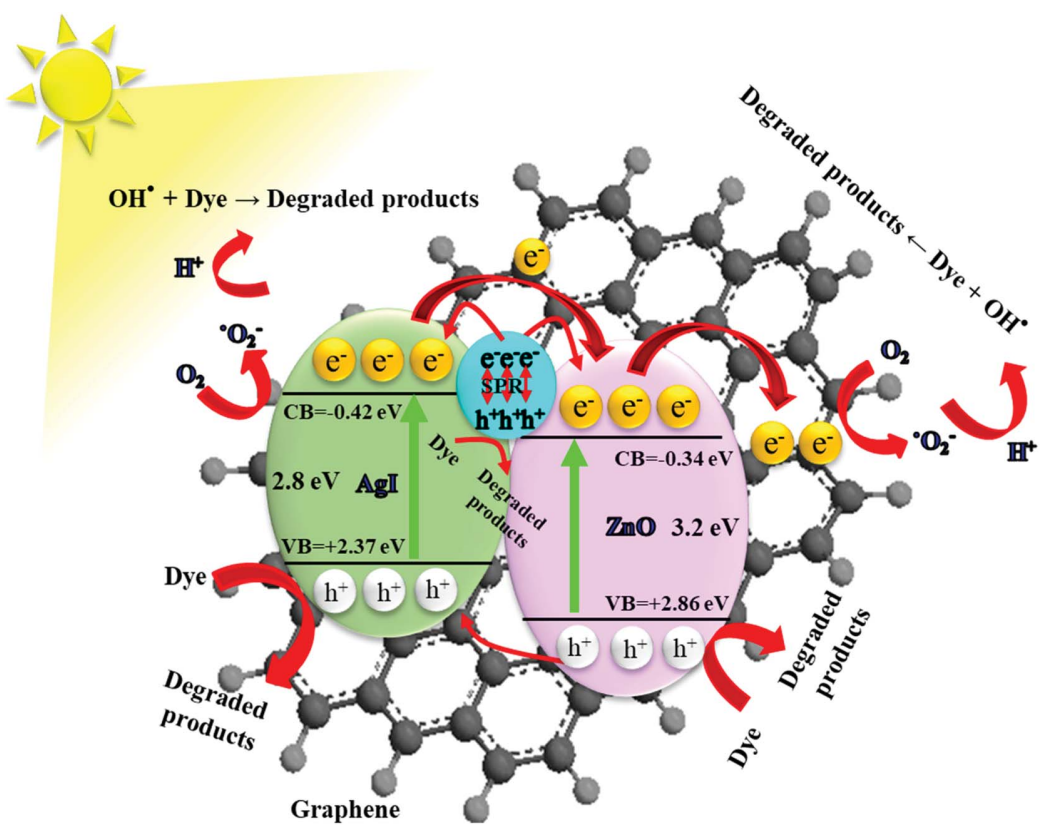

Fig. 12 Schematic diagram showing the energy band structure and possible $\mathrm{e}^{-} / \mathrm{h}^{+}$pair separation and charge carrier transport mechanisms in $\mathrm{ZnO} / \mathrm{GR} / \mathrm{Ag} / \mathrm{Agl}$ nanocomposites.

between GR, ZnO NPs, and Ag/AgI that favours interfacial charge transfer and prevents $\mathrm{e}^{-} / \mathrm{h}^{+}$recombination.

\section{Conclusion}

In summary, we have reported on a novel low-temperature chemical route, assisted by ultrasonication, to prepare $\mathrm{ZnO} /$ GR/Ag/AgI nanocomposites for state-of-the-art photocatalytic performance for degradation of $\mathrm{CR}$ and phenol. The prepared plasmonic nanocomposites have been characterized by various techniques to unravel their structural, morphological, electrical, optical and photocatalytic properties. The synthesized $\mathrm{ZnO} / \mathrm{GR} / \mathrm{Ag} / \mathrm{AgI}$ (20\%) nanocomposite exhibits the highest photodegradation efficiency $(90 \%)$ and the highest degradation constant rate $\left(308 \times 10^{-4} \mathrm{~min}^{-1}\right)$ for degradation of CR dye. The key advantages of the optimized nanocomposites include increased light absorption in the visible range due to a strong plasmonic resonance effect involving metallic $\mathrm{Ag}^{\mathrm{O}}$, and to the improved electronic transfer due to hybridization with graphene. In addition, the nanoparticles in the plasmonic nanocomposite facilitate an effective charge separation of the photoexcited charge carriers, mitigating $\mathrm{e}^{-} / \mathrm{h}^{+}$pair recombination. The scavenger experiments suggest that superoxide and hydroxyl radicals are responsible for the photodegradation of CR. The recycling test confirms a sufficient durability of the nanocomposite. We believe that the proposed plasmonic nanocomposites are promising for high photocatalytic performance applications such as removal of organic and inorganic pollutants, photoelectrochemistry and water oxidation.

\section{Conflicts of interest}

There are no conflicts of interest to declare.

\section{Acknowledgements}

The authors acknowledge the Department of Science and Technology (ITN) at Campus Norrköping, Linköping University, Sweden for partial financial support. The Knut and Alice Wallenberg Foundation is acknowledged for support of the Electron Microscopy Laboratory at Linköping University.

\section{References}

1 S. P. Lonkar, V. V. Pillai and S. M. Alhassan, Sci. Rep., 2018, 8, 13401.

2 D. Barreca, G. Carraro, A. Gasparotto, C. Maccato, T. Altantzis, C. Sada, K. Kaunisto, T.-P. Ruoko and S. Bals, Adv. Mater. Interfaces, 2017, 4, 1700161.

3 H. Zhang, G. Chen and D. W. Bahnemann, J. Mater. Chem., 2009, 19, 5089. 
4 H. Wang, X. Liu and S. Han, CrystEngComm, 2016, 18, 19331943.

5 S. Kumar, A. Kumar, A. Kumar, R. Balaji and V. Krishnan, ChemistrySelect, 2018, 3, 1919-1932.

6 A. Kumar, V. Sharma, S. Kumar, A. Kumar and V. Krishnan, Surf. Interfaces, 2018, 11, 98-106.

7 W. Zhong, S. Shen, S. Feng, Z. Lin, Z. Wang and B. Fang, CrystEngComm, 2018, 20, 7851-7856.

8 Y. Liu, S. Shen, J. Zhang, W. Zhong and X. Huang, Appl. Surf. Sci., 2019, 478, 762-769.

9 G. Liao, Y. Gong, L. Zhang, H. Gao, G.-J. Yang and B. Fang, Energy Environ. Sci., 2019, 12, 2080-2147.

$10 \mathrm{~J} . \mathrm{Xu}, \mathrm{Y}$. Cui, Y. Han, M. Hao and X. Zhang, RSC Adv., 2016, 6, 96778-96784.

11 R. Vinoth, P. Karthik, C. Muthamizhchelvan, B. Neppolian and M. Ashokkumar, Phys. Chem. Chem. Phys., 2016, 18, 5179-5191.

12 A. Kumar, K. L. Reddy, S. Kumar, A. Kumar, V. Sharma and V. Krishnan, ACS Appl. Mater. Interfaces, 2018, 10, 1556515581.

13 H. Sun and S. Wang, Energy Fuels, 2014, 28, 22-36.

14 S. Lee, D. A. Reddy and T. Kyu Kim, RSC Adv., 2016, 6, 3718037188.

15 J. Choi, D. A. Reddy, M. J. Islam, R. Ma and T. K. Kim, J. Alloys Compd., 2016, 688, 527-536.

16 N. Zhang, Y. Zhang and Y.-J. Xu, Nanoscale, 2012, 4, 57925813.

17 Q. Xiang, J. Yu and M. Jaroniec, Chem. Soc. Rev., 2012, 41, 782-796.

18 B. Li, T. Liu, Y. Wang and Z. Wang, J. Colloid Interface Sci., 2012, 377, 114-121.

19 B. Li and H. Cao, J. Mater. Chem., 2011, 21, 3346-3349.

20 E. Chalangar, H. Machhadani, S.-H. Lim, K. F. Karlsson, O. Nur, M. Willander and H. Pettersson, Nanotechnology 2018, 29, 415201.

21 Z. Tian, S. Bai, K. Cao and J. Li, Mater. Express, 2016, 6, 437443.

22 S. Y. Sawant and M. Hwan Cho, RSC Adv., 2015, 5, 9778897797.

23 K. L. Reddy, S. Kumar, A. Kumar and V. Krishnan, J. Hazard. Mater., 2019, 367, 694-705.

24 A. Kumar, K. Kumar and V. Krishnan, Mater. Lett., 2019, 245, 45-48.

25 D. A. Reddy, S. Lee, J. Choi, S. Park, R. Ma, H. Yang and T. K. Kim, Appl. Surf. Sci., 2015, 341, 175-184.

26 G. Li, Y. Wang and L. Mao, RSC Adv., 2014, 4, 53649-53661.

27 M. Pirhashemi and A. Habibi-Yangjeh, Ceram. Int., 2017, 43, 13447-13460.

28 N. Güy and M. Özacar, J. Photochem. Photobiol., A, 2019, 370, 1-11.

29 G. Liao, J. Fang, Q. Li, S. Li, Z. Xu and B. Fang, Nanoscale, 2019, 11, 7062-7096

30 W. Jiang, C. An, J. Liu, S. Wang, L. Zhao, W. Guo and J. Liu, Dalton Trans., 2013, 43, 300-305.

31 J. Yang, X. Zhao, X. Shan, H. Fan, L. Yang, Y. Zhang and X. Li, J. Alloys Compd., 2013, 556, 1-5.
32 M. Pirhashemi and A. Habibi-Yangjeh, J. Colloid Interface Sci., 2017, 491, 216-229.

33 B. Saravanakumar, R. Mohan and S.-J. Kim, Mater. Res. Bull., 2013, 48, 878-883.

34 D. Fu, G. Han, Y. Chang and J. Dong, Mater. Chem. Phys., 2012, 132, 673-681.

35 A. Monshi, M. R. Foroughi and M. R. Monshi, World J. Nano Sci. Eng., 2012, 02, 154-160.

36 B. Qiu, Y. Zhou, Y. Ma, X. Yang, W. Sheng, M. Xing and J. Zhang, Sci. Rep., 2015, 5, 8591.

37 S. Lu, H. Wang, J. Zhou, X. Wu and W. Qin, Nanoscale, 2017, 9, 1184-1192.

38 V. Etacheri, R. Roshan and V. Kumar, ACS Appl. Mater. Interfaces, 2012, 4, 2717-2725.

39 H. Alnoor, G. Pozina, V. Khranovskyy, X. Liu, D. Iandolo, M. Willander and O. Nur, J. Appl. Phys., 2016, 119, 165702.

40 D. K. Mishra, J. Mohapatra, M. K. Sharma, R. Chattarjee, S. K. Singh, S. Varma, S. N. Behera, S. K. Nayak and P. Entel, J. Magn. Magn. Mater., 2013, 329, 146-152.

41 H. Moussa, E. Girot, K. Mozet, H. Alem, G. Medjahdi and R. Schneider, Appl. Catal., B, 2016, 185, 11-21.

42 A. Kumar, L. Rout, L. S. K. Achary, S. Keshari Mohanty and P. Dash, New J. Chem., 2017, 41, 10568-10583.

43 K. Dai, J. Lv, L. Lu, C. Liang, L. Geng and G. Zhu, Mater. Chem. Phys., 2016, 177, 529-537.

44 H. N. Tien, V. H. Luan, L. T. Hoa, N. T. Khoa, S. H. Hahn, J. S. Chung, E. W. Shin and S. H. Hur, Chem. Eng. J., 2013, 229, 126-133.

45 C. Hu, T. Peng, X. Hu, Y. Nie, X. Zhou, J. Qu and H. He, J. Am. Chem. Soc., 2010, 132, 857-862.

46 M. R. Phillips, O. Gelhausen and E. M. Goldys, Phys. Status Solidi A, 2004, 201, 229-234.

47 S. Choi, M. R. Phillips, I. Aharonovich, S. Pornsuwan, B. C. C. Cowie and C. Ton-That, Adv. Opt. Mater., 2015, 3, 821-827.

48 V. Khranovskyy, V. Lazorenko, G. Lashkarev and R. Yakimova, J. Lumin., 2012, 132, 2643-2647.

49 B. Neppolian, A. Bruno, C. L. Bianchi and M. Ashokkumar, Ultrason. Sonochem., 2012, 19, 9-15.

50 J. Choi, D. A. Reddy and T. K. Kim, Ceram. Int., 2015, 41, 13793-13803.

51 D. Amaranatha Reddy, R. Ma, M. Y. Choi and T. K. Kim, Appl. Surf. Sci., 2015, 324, 725-735.

52 T. T. H. Tran, H. Kosslick, A. Schulz and Q. L. Nguyen, Adv. Nat. Sci.: Nanosci. Nanotechnol., 2017, 8, 015011.

53 L. Hou, H. Zhang and X. Xue, Sep. Purif. Technol., 2012, 84, 147-152.

54 A. L. Teel and R. J. Watts, J. Hazard. Mater., 2002, 94, 179189.

55 D. Amaranatha Reddy, J. Choi, S. Lee, R. Ma and T. Kyu Kim, RSC Adv., 2015, 5, 67394-67404.

56 Y. Li, X. Li, J. Li and J. Yin, Water Res., 2006, 40, 1119-1126.

57 N. Sedaghati, A. Habibi-Yangjeh, M. Pirhashemi and S. Vadivel, J. Photochem. Photobiol., A, 2019, 384, 112066.

58 S. Feizpoor, A. Habibi-Yangjeh, I. Ahadzadeh and K. Yubuta, Adv. Powder Technol., 2019, 30, 1183-1196.

59 A. H. Nethercot, Phys. Rev. Lett., 1974, 33, 1088-1091. 
60 H. Zhang, X. Lv, Y. Li, Y. Wang and J. Li, ACS Nano, 2010, 4, 380-386.

61 S. H. Hsieh, W. J. Chen and T. H. Yeh, Appl. Surf. Sci., 2015, 358, 63-69.

62 S. Song, B. Cheng, N. Wu, A. Meng, S. Cao and J. Yu, Appl. Catal., B, 2016, 181, 71-78.

63 P. Qiu, B. Park, J. Choi, M. Cui, J. Kim and J. Khim, J. Alloys Compd., 2017, 706, 7-15.

64 P. Dou, F. Tan, W. Wang, A. Sarreshteh, X. Qiao, X. Qiu and J. Chen, J. Photochem. Photobiol., A, 2015, 302, 17-22.
65 T. N. Reddy, J. Manna and R. K. Rana, ACS Appl. Mater. Interfaces, 2015, 35, 19684-19690.

66 X. Wu, L. Wen, K. Lv, K. Deng, D. Tang, H. Ye, D. Du, S. Liu and M. Li, Appl. Surf. Sci., 2015, 358, 130-136.

67 A. A. Ashkarran and B. Mohammadi, Appl. Surf. Sci., 2015, 342, 112-119.

68 E. Rokhsat and O. Akhavan, Appl. Surf. Sci., 2016, 371, 590595.

69 D. Xu, B. Cheng, S. Cao and J. Yu, Appl. Catal., B, 2015, 164, 380-388. 
PAPER

III 


\title{
Synthesis of Vertically Aligned ZnO Nanorods Using Sol-gel Seeding and Colloidal Lithography Patterning
}

\author{
Ebrahim Chalangar ${ }^{1,2}$, Omer Nur ${ }^{1}$, Magnus Willander ${ }^{1}$, Anders Gustafsson ${ }^{3}$ and Håkan Pettersson ${ }^{1,2,3^{*}}$ (D)
}

\begin{abstract}
Different $\mathrm{ZnO}$ nanostructures can be grown using low-cost chemical bath deposition. Although this technique is cost-efficient and flexible, the final structures are usually randomly oriented and hardly controllable in terms of homogeneity and surface density. In this work, we use colloidal lithography to pattern (100) silicon substrates to fully control the nanorods' morphology and density. Moreover, a sol-gel prepared ZnO seed layer was employed to compensate for the lattice mismatch between the silicon substrate and $\mathrm{ZnO}$ nanorods. The results show a successful growth of vertically aligned $\mathrm{ZnO}$ nanorods with controllable diameter and density in the designated openings in the patterned resist mask deposited on the seed layer. Our method can be used to fabricate optimized devices where vertically ordered $\mathrm{ZnO}$ nanorods of high crystalline quality are crucial for the device performance.
\end{abstract}

Keywords: Zinc oxide, Nanorod arrays, Vertical growth, Colloidal lithography, Nanofabrication, Sol-gel, Seed layer, Chemical bath deposition

\section{Introduction}

The ability to realize a desirable precise nanomaterial architecture with respect to dimensions, arrangement, and density of the nanostructures is crucial for most advanced nanodevices. One viable route to accomplish this is to combine a top-bottom patterning technique with a self-assembling bottom-up growth method.

Zinc oxide $(\mathrm{ZnO})$, one of the most widely studied semiconductors, shows an excellent potential for selfassembled optoelectronic nanodevices. Among the key properties of $\mathrm{ZnO}$ in this context can be mentioned, a simple growth procedure, a wide direct bandgap of 3.2$3.4 \mathrm{eV}$, a large exciton binding energy of $60 \mathrm{meV}$ and a high mechanical and thermal stability [1]. Different hightemperature growth techniques such as chemical vapor deposition (CVD) [2], pulsed laser deposition (PLD) [3]

\footnotetext{
*Correspondence: hakan.pettersson@hh.se

Department of Science and Technology, Physics, Electronics and Mathematics, Linköping University, Norrköping, Sweden

Full list of author information is available at the end of the article
}

and vapor-liquid-solid (VLS) growth [4], as well as lowtemperature growth techniques including electrodeposition [5] and chemical bath deposition (CBD) [6] have been used to grow a plenitude of $\mathrm{ZnO}$ nanostructures. Among all these growth techniques, CBD is more favorable due to its simplicity, cost-effectiveness and large-area applicability.

One-dimensional (1D) wurtzite $\mathrm{ZnO}$ nanorods $(\mathrm{ZnO}$ NRs) have attracted huge interest in the last two decades due to their interesting fundamental electronic and mechanical properties, as well as to their great promise for novel electronics [6], photonics [7], electrochemical [8] and clean-tech applications. The growth of ZnO NRs using CBD is highly dependent on the crystallographic surface quality of the substrate. The two most frequently used substrates to this date are i) single crystal lattice-matched substrates (single-crystalline $\mathrm{ZnO}$ [9], $\mathrm{Al}_{2} \mathrm{O}_{3}$ [10] and $\mathrm{GaN}$ [11]) with a thin heteroepitaxial $\mathrm{ZnO}$ film, and ii) non-epitaxial substrates precoated with a textured seed layer to provide suitable nucleation sites [12]. While single-crystalline substrates 
generally result in significantly higher NR growth quality, the high substrate cost limits their application. Conversely, the growth of NRs on low-cost non-epitaxial substrates, supplied with a textured polycrystalline seed layer, leads to a randomly oriented NR growth.

In many high-performance device applications, a precise engineering of the NR surface density, lateral ordering, and vertical alignment is of great importance. Growth of NRs by CBD offers several straightforward ways to engineer the NR architecture on the substrate by controlling growth parameters such as solution concentration [13], temperature [14], $\mathrm{pH}$ [15], and deposition duration. Unfortunately, changing any of these growth parameters typically also causes undesired changes in the NR growth result. For this reason, up to now, substrate patterning is the most effective way to control the position, density and alignment of $\mathrm{ZnO}$ NRs. In the vast majority of reported studies, patterning of the substrate includes the following steps: substrate preparation and cleaning, deposition of a mask resist layer, opening holes in the resist layer by lithography for selective area growth, and CBD growth of NRs in the holes.

During the past decade, various efforts have been made to grow ordered vertical $\mathrm{ZnO} \mathrm{NR}$ arrays. In the majority of these studies, using the same CBD technique, the authors have focused on two key processing steps: preparation of appropriate substrates, and usage of different patterning techniques, e.g., electron-beam lithography (EBL), laser interference lithography (LIL), and nanoimprint lithography (NIL). Wang et al. demonstrated non-epitaxial growth of vertically aligned $\mathrm{ZnO} \mathrm{NRs}$ on EBL-patterned polycrystalline $\mathrm{ZnO}$-coated $\mathrm{Si}$ and $\mathrm{GaN}$ substrates [11]. Later, they reported on perfectly aligned heteroepitaxial $\mathrm{ZnO} \mathrm{NR}$ growth on $\mathrm{GaN}$ substrates patterned by LIL [16]. In an effort to replace the expensive $\mathrm{ZnO}$ and $\mathrm{GaN}$ substrates with low-cost Si or glass substrates, they successfully used a 30- $\mu \mathrm{m}$-thick textured $\mathrm{ZnO}$ layer as a flat (0001) $\mathrm{ZnO}$ seed layer. In a series of articles, Volk et al. successfully performed homoepitaxial growth of $\mathrm{ZnO}$ NRs on single-crystalline $\mathrm{ZnO}$ substrates patterned by EBL [10, 17-19]. The impact of different patterned substrates [10], a Zn-terminated versus O-terminated surface of the $\mathrm{ZnO}$ substrate [17], a sputtered polycrystalline $\mathrm{ZnO}$ thin film [18], and a $\mathrm{ZnO}$ seed layer deposited by atomic layer deposition (ALD) [19] on the quality of CBD-grown $\mathrm{ZnO}$ NRs have been deeply investigated. In a different approach, PLD was used for heteroepitaxial growth of a $\mathrm{ZnO}$ seed layer on a singlecrystalline $\mathrm{Al}_{2} \mathrm{O}_{3}$ (0001) substrate, followed by NIL patterning [20]. More details about the $\mathrm{Zn}$ concentration window in CBD and its effect on the $\mathrm{ZnO}$ NR morphology were discussed in the same article. Selective area growth of $\mathrm{ZnO} \mathrm{NRs}$ on different substrates, including $\mathrm{Si}$
(111), GaAs (111) and InP (111), using EBL patterning and electrochemical deposition has also been reported [21].

In all the reviewed references, the growth of $\mathrm{ZnO}$ NRs on a single-crystalline $\mathrm{ZnO}$ substrate results in the highest crystal quality, while a coating with a thin polycrystalline $\mathrm{ZnO}$ layer on other expensive single-crystalline substrates leads to excellent vertically aligned $\mathrm{ZnO}$ NR growth. Employing sophisticated patterning techniques, e.g., EBL, for selective growth of $\mathrm{ZnO} \mathrm{NRs}$ also has an essential impact on the final product cost and, consequently, on the potential application. To overcome demanding complexity issues related to pattern definition and expensive substrates in the fabrication of vertically aligned $\mathrm{ZnO} \mathrm{NR}$ arrays, we propose to combine a simply prepared, highly uniform thin $\mathrm{ZnO}$ NP film on $\mathrm{Si}$ substrates with colloidal lithography $(\mathrm{CL})$ patterning.

A sol-gel technique, described in [22], can be used to prepare a highly uniform, dense and thin seed layer of polycrystalline $\mathrm{ZnO}$ NPs on virtually any substrate, including $\mathrm{Si}$ or glass. In contrast to the spin-coating of a $\mathrm{ZnO}$ NP dispersion on a substrate, which usually leads to non-continuous dispersed islands of NP clusters [23], the sol-gel method results in a thin (tens of nm) continuous and highly uniform layer of ZnO NPs. For selective area CBD growth, a polymer resist layer is subsequently spin-coated on the $\mathrm{ZnO}$ seed layer and patterned by CL. Besides being a low-cost lithography method, CL is also suitable for large wafer-scale area patterning, high throughput, and compatibility with any substrate, offering excellent control over feature size and pitch [24-26].

Figure 1 shows a schematic of the processing and growth steps used in the present work. After dip-coating the Si substrate with a ZnO NP seed layer and spin-coating with a resist, respectively, a monolayer of polystyrene nanobeads (PS-NBs) with a diameter of $140 \mathrm{~nm}$ was deposited on the resist mask and covered by a thermally evaporated $30 \mathrm{~nm}$ thick $\mathrm{Al}$ film. After evaporation, the Al-coated PS-NBs were removed by tape stripping, leaving behind open nanoholes in the Al film. Dry etching of the resist in the holes down to the $\mathrm{ZnO} \mathrm{NP}$ seed layer finalized the preparation of the growth mask. After $\mathrm{CL}, \mathrm{CBD}$ was used to grow vertically aligned $\mathrm{ZnO} \mathrm{NRs}$ with well-controlled diameter, length and surface density. While CL and CBD have previously been deployed to grow $\mathrm{ZnO} \mathrm{NRs}$ on micrometer-sized areas on ITO glass substrates, resulting in large uncontrolled batches of grown NRs [27], the present study is, to the best of our knowledge, the first demonstrated selective growth of single vertically aligned $\mathrm{ZnO}$ NRs in CL-patterned masks on sol-gel prepared Si substrates. The results show a uniform distribution of nanoholes on a large wafer-scale area 


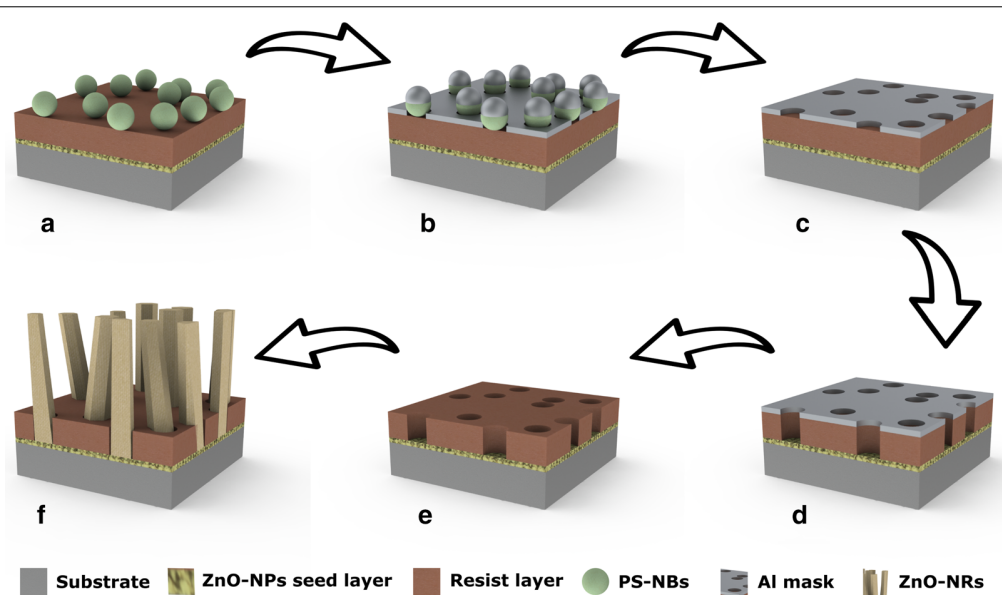

Fig. 1 Schematic of the fabrication process steps to synthesize vertically aligned ZnO NR arrays. a A substrate coated with a ZnO NP seed layer, a polymer resist layer and PS-NBs. b Thermal evaporation of an Al mask. c Removing the Al-coated PS-NBs by tape stripping. $\mathbf{d}$ Dry etching of the resist. e Wet etching of the Al mask. $\mathbf{f}$ Selective $\mathrm{ZnO}$ NR growth in the holes of the patterned resist template

and a homogeneous growth of vertical ZnO NRs using a facile and controllable CBD technique.

\section{Methods}

\section{Materials}

All the chemicals used in this work were purchased from Sigma-Aldrich and used without any further purification. The suspension of PS-NBs with a mean diameter of $140 \pm 5 \mathrm{~nm}$ in water was purchased from microParticles GmbH, Germany.

\section{Preparation of the ZnO NP Seed Layer}

The ZnO NP sol-gel solution was prepared according to [22] by dissolving $8.25 \mathrm{~g}$ of zinc acetate $\left(\mathrm{Zn}\left(\mathrm{CH}_{3} \mathrm{COO}\right)_{2} \cdot 2 \mathrm{H}_{2} \mathrm{O}\right)$ and $2.26 \mathrm{ml}$ of monoethanolamine (ethanolamine) in $100 \mathrm{ml}$ of pure ethanol with final concentrations of $375 \mathrm{mM}$. The solution was stirred on a hotplate at $60{ }^{\circ} \mathrm{C}$ for $10 \mathrm{~h}$ and then at room temperature overnight. A two-inch, highly doped n-type Si (100) wafer was cleaned by sequential ultrasonication in acetone, isopropanol, and DI water, followed by drying on a hotplate at $120{ }^{\circ} \mathrm{C}$ for $5 \mathrm{~min}$. The cleaned $\mathrm{Si}$ substrate was dipped into the $\mathrm{ZnO} \mathrm{NP}$ sol-gel solution and pulled out at a constant speed of $30 \mathrm{~mm} / \mathrm{s}$ using a computer-controlled dip-coater. Finally, it was annealed on a hotplate at $300{ }^{\circ} \mathrm{C}$ for $10 \mathrm{~min}$ to remove the residual organics and improve the
ZnO NP crystallinity. The dip-coating and the annealing steps were repeated two times to increase the layer homogeneity and surface coverage.

\section{Patterning the Seeded Sample by $\mathrm{CL}$}

The CL was carried out on a double-layer resist, consisting of a sacrificial PMMA (poly(methyl methacrylate), $M_{W}=950 \mathrm{k}$ ) electron-beam resist and a positive MICROPOSIT S1805 photoresist. First, $0.3 \mathrm{ml}$ of a $4 \mathrm{wt} \%$ solution of PMMA in toluene was spin coated on the seeded sample at $4000 \mathrm{rpm}$ for $30 \mathrm{~s}$, followed by soft baking at $170{ }^{\circ} \mathrm{C}$ for $10 \mathrm{~min}$. Subsequently, $0.3 \mathrm{ml}$ of S1805 was spin coated on the sample at $4000 \mathrm{rpm}$ for $30 \mathrm{~s}$, followed by soft baking at $110{ }^{\circ} \mathrm{C}$ for $90 \mathrm{~s}$ and hard baking at $145{ }^{\circ} \mathrm{C}$ for $5 \mathrm{~min}$. Next, the sample was treated by UV-ozone for $5 \mathrm{~min}$, improving the surface hydrophilicity, and then $1 \mathrm{ml}$ of a $0.2 \mathrm{wt} \%$ solution of PDDA (poly(diallyldimethylammonium), $\quad M_{W}=(200-350) \mathrm{k}$ ) in DI water was pipetted on the sample surface. After $1 \mathrm{~min}$. of settling, the sample was thoroughly rinsed with DI water and blow-dried with $\mathrm{N}_{2}$. The positively charged monolayer of PDDA guarantees electrostatic adhesion of negatively charged PS-NBs to the surface in the next step. Afterward, $1 \mathrm{ml}$ of a $0.1 \mathrm{wt} \%$ suspension of PS-NBs in DI water was dropped on the sample surface. After $1 \mathrm{~min}$. of settling, the sample was gently rinsed with DI water, and blow-dried with $\mathrm{N}_{2}$. A $30 \mathrm{~nm}$ thick $\mathrm{Al}$ layer was thermally evaporated on the beaded sample as a metal etch mask. 
After evaporation, the Al-coated PS-NBs were removed by tape stripping using acrylic silicon-free Ultron 1009R tape (Fig. 1c). Reactive-ion etching (RIE, pressure 150 mTorr, $\mathrm{O}_{2}$ flow $40 \mathrm{sccm}$, RF power $60 \mathrm{~W}$ and etching time $90 \mathrm{~s}$ ) was employed to etch the resist layer down to the $\mathrm{ZnO}$ NP seed layer (Fig. 1d). Finally, to wet-etch the Al metal mask (Fig. 1e) without etching the exposed $\mathrm{ZnO}$ NP seed layer, a particular solution of potassium hydroxide $(\mathrm{KOH})$ and potassium hexacyanoferrate III $\left(\mathrm{K}_{3} \mathrm{Fe}(\mathrm{CN})_{6}\right)$ in DI water with a concentration of $30 \mathrm{mM}$ and $50 \mathrm{mM}$ respectively was used [28]. The wet etching was conducted at room temperature for $1 \mathrm{~min}$. Scanning electron microscopy (SEM) and atomic force microscopy (AFM) images of the fabrication steps are provided in Additional file 1: Figures S1 and S2.

\section{CBD of ZnO NRs on the CL-Patterned Sample}

A solution of $50 \mathrm{mM}$ zinc nitrate hexahydrate $\left(\mathrm{Zn}\left(\mathrm{NO}_{3}\right)_{2} \cdot 6 \mathrm{H}_{2} \mathrm{O}\right)$ and $50 \mathrm{mM}$ hexamethylenetetramine (HMT) in $100 \mathrm{ml}$ DI water was prepared to grow the $\mathrm{ZnO}$ NRs. The patterned seeded substrates were kept upside down in the growth solution for $2 \mathrm{~h}$ at $95^{\circ} \mathrm{C}$ in an oven. After the growth was completed, the samples were cooled to room temperature, removed from the solution, and rinsed with DI water (Fig. 1f).

\section{Results and Discussion ZnO Seed Layer}

The growth of well-aligned vertical $\mathrm{ZnO}$ NRs with high crystallinity relies on the quality of the seed layer. For this, we developed smooth seed layers with large grain sizes and optimal crystal orientation. The prepared $\mathrm{ZnO}$ NP seed layer was investigated with respect to surface roughness and crystal structure using AFM and X-ray diffraction (XRD) analysis. The AFM images in Fig. 2 show significant improvement in the homogeneity and the smoothness of the seed layer after repeating the dipcoating, as explained above. After a single-step dip-coating of a Si substrate in the sol-gel solution, a $21 \pm 5 \mathrm{~nm}$ thick $\mathrm{ZnO}$ NP layer with an RMS roughness of $1.2 \mathrm{~nm}$ (Fig. 2a) was formed on the Si surface. Repeating the dipcoating resulted in a smoother $40 \pm 5 \mathrm{~nm}$ thick $\mathrm{ZnO} \mathrm{NP}$ layer with an RMS roughness of $0.9 \mathrm{~nm}$ at a grain boundary (Fig. 2b). Each dip-coating step was followed by an annealing treatment at $300{ }^{\circ} \mathrm{C}$ for $10 \mathrm{~min}$ that sintered the NPs together into bigger crystal grain sizes.

Three factors mainly influence the crystal grain boundaries observed in AFM images: (1) the thickness of the $\mathrm{ZnO}$ seed layer that is proportional to the number of dip-coating steps, (2) the temperature and the duration of subsequent annealing steps, and (3) impurities or dopants present in the crystal structure. It has been shown that larger grain sizes are achieved by increasing the thickness of the $\mathrm{ZnO}$ layers [29, 30]. Also, annealing at a higher temperature and for a longer time leads to better sintering of the small particles and increased grain sizes. In addition, unintentional impurities present during the synthesis process, or added dopants to the $\mathrm{ZnO}$ seed layer results in smaller grain sizes [31]. Although our samples are not intentionally doped, the presence of monoethanolamine in the sol-gel solution most likely introduces impurities during the annealing, causing smaller grain sizes. The crystal grain zones are comparably larger than the $140 \mathrm{~nm}$ diameter PS-NBs used for the CL. Consequently, the probability of ending up with grown $\mathrm{ZnO} \mathrm{NRs}$ in holes on top of a single crystal grain without a grain boundary is increased.

To grow vertically aligned $\mathrm{ZnO}$ NRs, a (002)-oriented $\mathrm{ZnO}$ seed layer is desirable. The XRD results in Fig. 3 show the crystal structure and orientation of one- and

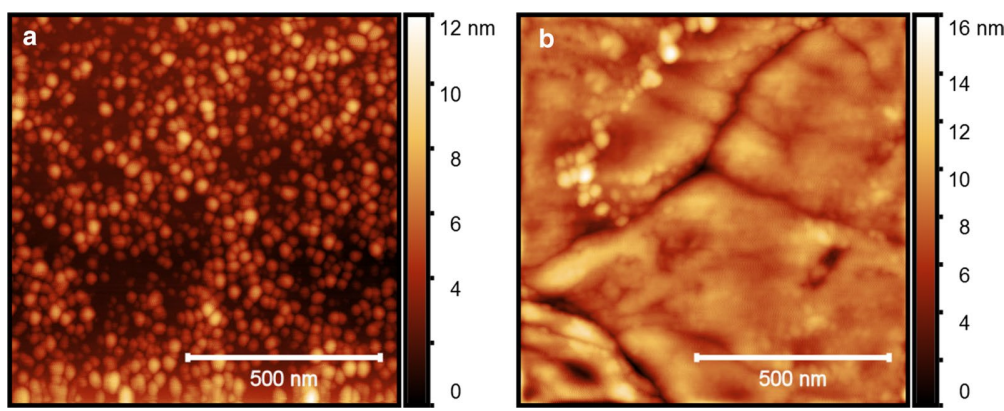

Fig. 2 AFM images of ZnO NP seed layers prepared on top of a Si wafer by a sol-gel method after $\mathbf{a}$ one- and $\mathbf{b}$ two-step dip-coatings, respectively, followed by an annealing step at $300^{\circ} \mathrm{C}$ after each coating. A polynomial background has been subtracted from the AFM images 


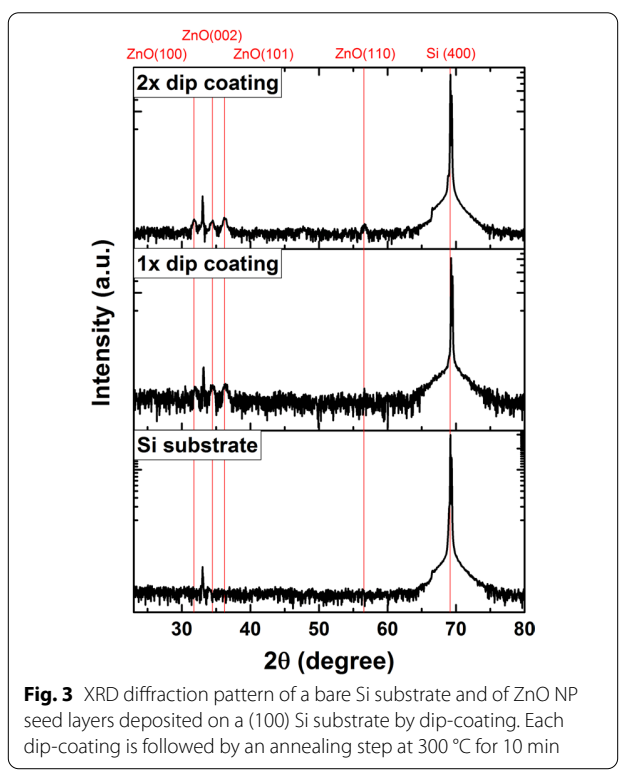

two-step dip-coated seed layers. In both samples, the polycrystalline $\mathrm{ZnO}$ seed layer exhibits crystal plane orientations of (100), (002) and (101). The self-forming process of polycrystalline ZnO NPs has previously been studied in more detail $[5,32]$. Applying the second dipcoating and annealing treatment increased the ratio between the integrated XRD peak areas produced by the (100) and (002) planes in agreement with a previous report [29]. Although repeating the dip-coating process improves the seed layer smoothness and grain size, the crystal structure thus becomes less favorable for $\mathrm{ZnO} N R$ growth by the increased presence of (100) planes.

\section{CL patterning and ZnO NRs Growth}

The deposition of the $\mathrm{ZnO}$ seed layers was directly followed by CL-patterning of selected areas. Figure 4a shows an SEM image of a CL-patterned resist layer after RIE etching and Al removal. A uniform, large-area CL patterning is clearly demonstrated on the $\mathrm{ZnO}$-seeded Si substrate, with a nanohole surface density of 4.2 nanohole $/ \mu \mathrm{m}^{2}$ and a diameter distribution centered around $190 \mathrm{~nm}$ (Fig. 4b). The final nanohole diameter was found to be $36 \%$ larger than the diameter of the PS-NBs, which is attributed to the $90 \mathrm{~s}$ RIE of the resist layer. The diameter of the nanoholes can simply be tuned by choosing a different PS-NB size. Here, we chose an optimized $140 \mathrm{~nm}$ bead size to ensure a single $\mathrm{ZnO}$ NR growth in each nanohole by CBD. Using a smaller PS-NB size makes the CBD growth more difficult due to the insufficient flow of the growth solution into the hydrophobic patterned resist. Using larger diameter PS-NBs instead
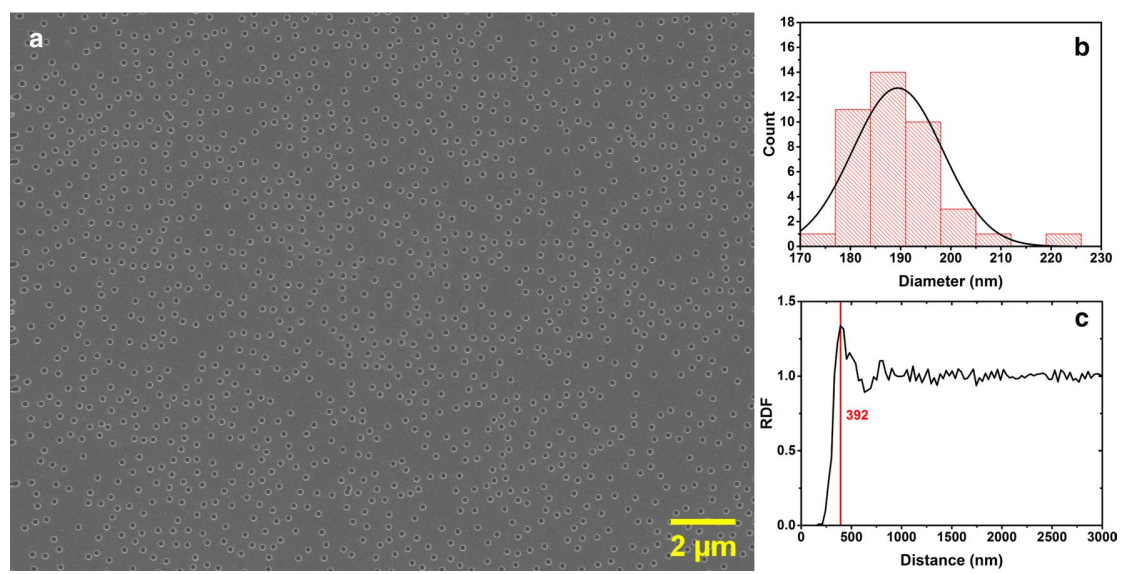

Fig. 4 a SEM image of a CL-patterned resist layer using 140-nm-diameter PS-NBs on a ZnO NP-seeded Si substrate, after RIE etching and Al wet etching. $\mathbf{b}$ Distribution of the diameter of the etched nanoholes in the resist layer. $\mathbf{c}$ The radial distribution function of the patterned nanoholes, with an average nanohole neighboring separation of $392 \mathrm{~nm}$ 
results in undesired growth of clusters of $\mathrm{ZnO}$ NRs in each nanohole $[21,27,33]$.

In addition to the nanohole feature size, the pitch of the pattern can also be tuned by controlling the average distance between the dispersed PS-NBs. Negatively charged PS-NBs are distributed on the surface by electrostatic net repulsive forces, which can be controlled by screening the electrostatic repulsion forces between the PSNBs. This has been done by adding a controlled amount of salt into the PS-NB suspension, as previously reported at length in literature $[25,26]$. Here we instead considered the effect of PS-NB concentration on the nanohole surface distribution. In addition to the $0.1 \mathrm{wt} \%$ PS-NB suspension mentioned above, three lower concentrations of $0.02 \mathrm{wt} \%, 0.01 \mathrm{wt} \%$ and $0.003 \mathrm{wt} \%$ were used to fabricate CL-patterned samples. Additional file 1: Figure S4 shows that a reduction of the NB concentration to the mentioned values results in nanohole surface densities of $3.2,1.5$, and 0.4 nanohole $/ \mu \mathrm{m}^{2}$, respectively. Interestingly, the radial distribution function (RDF) of the nanoholes for the different samples (Fig. 4c and Additional file 1: S4d) shows a short-range order in the distribution of the nanoholes due to the net force between the PS-NBs in the CL process. From the extracted primary peak positions for different PS-NBs concentrations, it was extracted that the average nanohole separation to its neighbor counterintuitively reduces with decreasing PS-NB concentration to $392 \mathrm{~nm}, 374 \mathrm{~nm}, 336 \mathrm{~nm}$, and $298 \mathrm{~nm}$, respectively. From this can be concluded that lower PS-NB concentrations result in a less uniform nanohole distribution, as visible in the SEM images in Additional file 1: Figure S4.

To investigate the growth mechanism of $\mathrm{ZnO}$ NRs on the CL-patterned substrates more in detail, a study of the growth rate versus growth time was carried out. Figure $5 \mathrm{a}$, and $5 \mathrm{~b}$ show the early stages of the $\mathrm{ZnO} \mathrm{NR}$ growth after $5 \mathrm{~min}$ and $25 \mathrm{~min}$, respectively. In the beginning, the exposed opened seeded holes form a wetting layer template for the growth solution with random crystal orientation, providing nucleation sites for $\mathrm{CBD}$ of $\mathrm{ZnO}$ NRs. Multiple ZnO NRs epitaxially grow from the nuclei sites along random directions (Fig. 5a). The nanohole walls restrict the growth of NRs with large deviation angles, and only those few NRs with a near-vertical direction will continue to grow, as seen in Fig. 5b.

By continuing the CBD process, the nanoholes are filled up by short off-direction NR stubs and only a few NRs grow out from the patterned resist layer. At a high enough growth temperature $\left(95{ }^{\circ} \mathrm{C}\right.$ in our experiment), a few near-vertical $\mathrm{ZnO} \mathrm{NRs}$ in each nanohole merge together, forming a single NR growing out from each opening in agreement with a previous report [11]. The
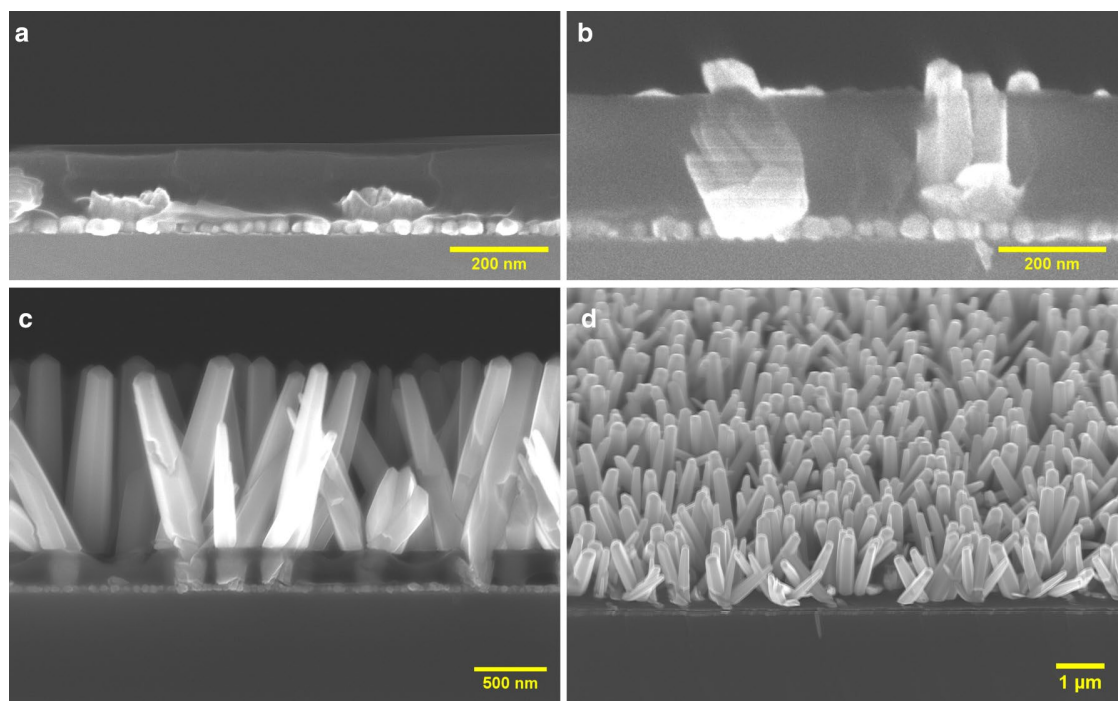

Fig. 5 Cross-sectional SEM images of CBD-grown ZnO NRs on CL-patterned ZnO NP seed layers on (100) Si substrates after a growth time of a 5 min, b 25 min, $\mathbf{c} 2$ h and $\mathbf{d} 2$ h at $45^{\circ}$ tilt 


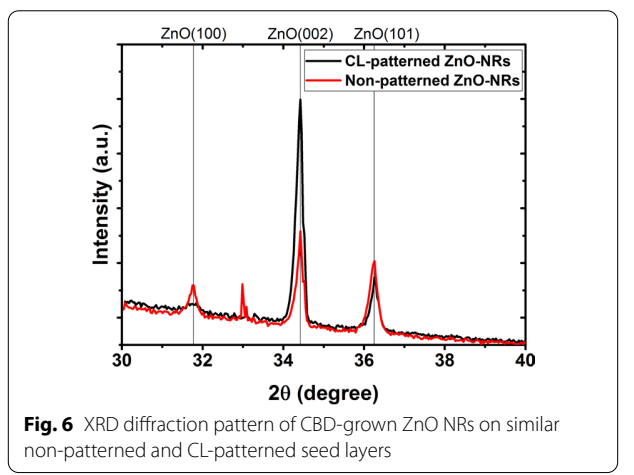

optimal ZnO NR array obtained on a large wafer-scale sample is shown in Fig. $5 \mathrm{c}$ and $5 \mathrm{~d}$.

To quantify the vertical NR alignment, we performed an XRD analysis of ZnO NR growth on similar nonpatterned and CL-patterned seed layers. Figure 6 shows that the $\mathrm{ZnO}(002)$ reflection is dominant for the CLpatterned sample, indicating a better $\mathrm{ZnO} \mathrm{NR}$ c-axis alignment. In contrast, the non-patterned sample shows more pronounced $\mathrm{ZnO}$ (100) and (101) reflections resulting from a poor vertical alignment. In addition, a statistical analysis of the SEM images of CL-patterned (Fig. 5c) and non-patterned samples (Additional file 1: Figure S3a) was carried out. The distributions of the deviation angle from the surface normal in Additional file 1: Figures S3b and $\mathrm{S} 3 \mathrm{c}$ show a mean deviation angle of $18^{\circ}$ for the nonpatterned sample and $13^{\circ}$ for the CL-patterned sample, respectively. This result provides further support for the conclusion that ZnO NR growth on CL-patterned samples yields better vertical alignment compared to nonpatterned samples.

Two additional CL-patterned samples, using 107-nmand 320-nm-diameter PS-NBs, were prepared and examined by SEM (images added in Additional file 1: Figure S5). The small 107-nm-diameter PS-NBs resulted in a poor and inhomogeneous CBD of $\mathrm{ZnO}$ NRs while the bigger 320-nm-diameter PS-NBs led to rather uniform, but multiple star-shaped and randomly aligned $\mathrm{ZnO}$ NRs. This result strongly indicates that choosing an optimal nanohole size, depending on the diameter of the $\mathrm{NRs}$, is crucial to grow a single, vertically aligned $\mathrm{ZnO}$ NR in each nanohole.

A chemical analysis of the final optimal CBD-grown $\mathrm{ZnO}$ NR array was done using dispersive $\mathrm{X}$-ray spectroscopy (EDS). The spatial EDS maps (Additional file 1: Figure S6) show clear presence of the expected elements $\mathrm{O}$, $\mathrm{Zn}, \mathrm{Si}$ and $\mathrm{C}$. The thin $\mathrm{ZnO}$ seed layer was not identified due to the spatial resolution limit.

Finally, in Fig. 7, we show spatially resolved cathodoluminescence mapping of a single ZnO NR. The cathodoluminescence study was performed in a dedicated SEM at room temperature, operating at $5 \mathrm{keV}$ with a probe current of $25 \mathrm{pA}$. The luminescence was recorded by a CCD detector in hyperspectral mode, where a full spectrum is recorded in each pixel of the images. An average cathodoluminescence intensity spectra (Fig. 7a), and

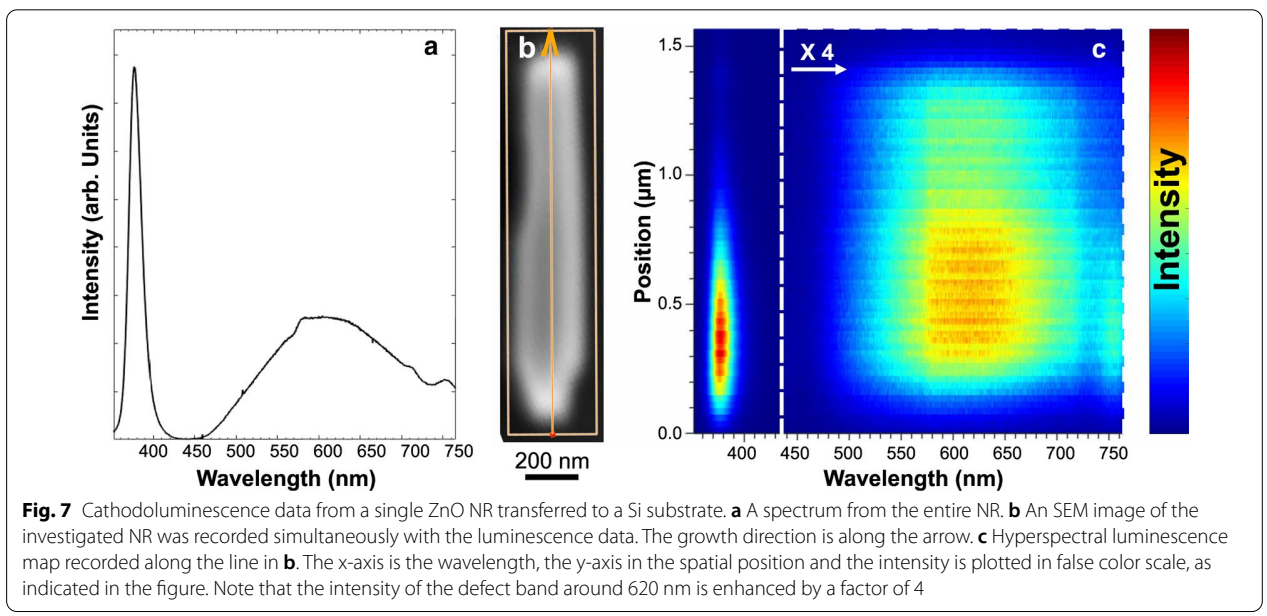


hyperspectral maps along a line (Fig. 7b), is presented as a false-color image in Fig. 7c. Interestingly, the strong near-band-edge emission, with a peak at $380 \mathrm{~nm}$, was observed in the bottom segment of the NR. It becomes weaker further up, where instead strong deep-level recombination results in a broad emission band with a 620 -nm peak position. The overall top view cathodoluminescence in Additional file 1: Figure S7, also shows the two spectral features luminescence in a larger area.

It is well known that the deep-level emission is due to the native point-defect levels of $\mathrm{Zn}$ and $\mathrm{O}$ vacancies in the crystal structure. The non-uniform spatial distribution of the deep-level emission thus indicates an inhomogeneous defect distribution along the NRs, with higher defect density in stronger emission regions. This local defect variation can be attributed to the change of the growth parameters, e.g., precursor concentrations, during the CBD process, as previously reported in the literature $[34,35]$.

As frequently reported in the literature, the vertical growth of $\mathrm{ZnO}$ NRs is claimed only by demonstrating top-view SEM images or integrated XRD diffraction patterns. But more accurate cross-sectional imaging typically shows that growth on non-patterned substrates, in the early phase, results in a compact, textured $\mathrm{ZnO}$ layer with vertical alignment at the top of the short NRs [36]. The individual NRs are more or less indistinguishable from one another, leaving no open paths to the substrate (Additional file 1: Figure S3a). In contrast, growth on patterned substrates results in NR arrays with open space in between. Evidently, the nanofabrication technique demonstrated here is capable of synthesizing a bottom-up, density-controlled, substrate-independent, and selective growth of single $\mathrm{ZnO}$ NRs with high quality. Because of the intrinsic behavior of epitaxial growth on a polycrystalline seed layer, the final NR arrays do not exhibit a perfect vertical alignment. However, a significant improvement in vertical alignment is readily observed compared to non-patterned samples (Additional file 1: Figure S3a). Further in-depth investigations are needed to further improve and control the critical crystal orientation of the seed layer.

\section{Conclusions}

In summary, we realized an almost vertical growth of ZnO NRs on CL-pattered (100) Si substrates, precoated with a $\mathrm{ZnO} \mathrm{NP}$ seed layer. The seed layer was uniformly deposited using a sol-gel technique by dip-coating the $\mathrm{Si}$ substrates in the sol-gel solution. Our findings show that two-step dip-coating enhances the smoothness and the crystal grain size of the seed layer, leading to a better NR alignment. Moreover, a selective area nanohole patterned resist template with tunable diameter and pitch was fabricated on the seeded substrates using CL. Subsequently, we grew a density-controlled array of single $\mathrm{ZnO}$ NRs in the patterned nanoholes by CBD and investigated them by XRD and cathodoluminescence with respect to crystal quality. Also, the $\mathrm{ZnO} \mathrm{NR}$ growth stages were studied by SEM after different growth time intervals. Our demonstrated nanofabrication technique, offering simplicity, uniformity over large wafer-scale areas, and controllable growth of vertical $\mathrm{ZnO}$ NRs can be used to fabricate high-performance devices.

\section{Abbreviations}

ZnO NRs: Zinc oxide nanorods; NPs: Nanoparticles; CBD: Chemical bath deposition; CL: Colloidal lithography; PS-NBs: Polystyrene nanobeads; DI: Deionized water; RIE: Reactive-ion etching; SEM: Scanning electron microscopy; EDS: Energy-dispersive X-ray spectroscopy; AFM: Atomic force microscopy; XRD: $X$-ray diffraction; RMS: Root mean square; RDF: Radial distribution function.

\section{Supplementary Information}

The online version contains supplementary material available at https://doi. org/10.1186/s11671-021-03500-7.

Additional file 1: Figure S1: SEM images after different colloidal lithography steps. Figure S2: AFM image and surface line profile of dry-etched resist layer. Figure S3: SEM image of CBD-grown ZnO-NRs and vertical alignment distribution plots. Figure S4: Nanohole surface distributions on CL-patterned resist layers. Figure S5: SEM images of CBD-grown ZnO-NRs on CL-patterned ZnO-NP seed layers. Figure S6: EDS mapping of CBDgrown ZnO-NRs. Figure S7: Top-view cathodoluminescence mapping of ZnO-NR sample in Fig. S6.

Acknowledgements

Not applicable.

\section{Authors' Contributions}

EC and HP conceived the research idea and analyzed the data. EC prepared and characterized all samples and wrote the original draft. AG conducted the cathodoluminescence measurements. HP supervised the research and acquired funding. HP, AG, ON and MW read and revised the manuscript and discussed the research results. All authors read and approved the final manuscript.

\section{Funding}

Open access funding provided by Lund University. This research was funded by the ÅForsk Foundation (project number 19-725), with additional financial support from Halmstad University, Linköping University and Lund University. The detector used for the cathodoluminescence was financed by the Crafoord Foundation.

\section{Availability of Data and Materials}

All data relevant for the reproduction of the results presented in this work are included in this published article or in its supplementary information (SI) file.

\section{Declarations}

Competing interests

The authors declare that they have no competing interests.

\section{Author details}

'Department of Science and Technology, Physics, Electronics and Mathematics, Linköping University, Norrköping, Sweden. ${ }^{2}$ School of Information Technology, Halmstad University, 30118 Halmstad, Sweden. ${ }^{3}$ Solid State Physics and NanoLund, Lund University, Box 118, 22100 Lund, Sweden. 
Received: 20 November 2020 Accepted: 21 February 2021 Published online: 12 March 2021

\section{References}

References
1. Kołodziejczak-Radzimska A, Jesionowski T (2014) Zinc oxide-from synthesis to application: a review. Materials 7:2833-2881. https://doi. org/10.3390/ma7042833

2. Müller R, Huber F, Gelme O et al (2019) Chemical vapor deposition growth of zinc oxide on sapphire with methane: initial crystal formation process. Cryst Growth Des 19:4964-4969. https://doi.org/10.1021/acs. cgd. 9 b00181

3. Rahman $F$ (2019) Zinc oxide light-emitting diodes: a review. OE 58:010901. https://doi.org/10.1117/1.OE.58.1.010901

4. Gomez JL, Tigli O (2013) Zinc oxide nanostructures: from growth to application. J Mater Sci 48:612-624. https://doi.org/10.1007/s1085 3-012-6938-5

5. Skompska M, Zarębska K (2014) Electrodeposition of ZnO nanorod arrays on transparent conducting substrates - a review. Electrochim Acta 127:467-488. https://doi.org/10.1016/jelectacta.2014.02.049

6. Xu S, Wang ZL (2011) One-dimensional ZnO nanostructures: solution growth and functional properties. Nano Res 4:1013-1098. https://doi. org/10.1007/s12274-011-0160-7

7. Cui J (2012) Zinc oxide nanowires. Mater Charact 64:43-52. https://doi. org/10.1016/j.matchar.2011.11.017

8. Adam RE, Chalangar E, Pirhashemi M et al (2019) Graphene-based plasmonic nanocomposites for highly enhanced solar-driven photocatalytic activities. RSC Adv 9:30585-30598. https://doi.org/10.1039/C9RA06273D

9. Consonni V, Sarigiannidou E, Appert E et al (2014) Selective area growth of well-ordered ZnO nanowire arrays with controllable polarity. ACS Nano 8:4761-4770. https://doi.org/10.1021/nn500620t

10. Erdélyi R, Nagata T, Rogers DJ et al (2011) Investigations into the impact of the template layer on $\mathrm{ZnO}$ nanowire arrays made using low temperature wet chemical growth. Cryst Growth Des 11:2515-2519. https://doi. org $/ 10.1021 / \mathrm{cg} 2002755$

11. Xu S, Wei Y, Kirkham M et al (2008) Patterned growth of vertically aligned $\mathrm{ZnO}$ nanowire arrays on inorganic substrates at low temperature withou catalyst. J Am Chem Soc 130:14958-14959. https://doi.org/10.1021/ja806 952j

12. Greene LE, Law M, Tan DH et al (2005) General route to vertical ZnO nanowire arrays using textured $\mathrm{ZnO}$ seeds. Nano Lett 5:1231-1236. https ://doi.org/10.1021/nl050788p

13. Yilmaz C, Unal U (2016) Effect of $\mathrm{Zn}(\mathrm{NO} 3) 2$ concentration in hydrothermal-electrochemical deposition on morphology and photoelectrochemical properties of ZnO nanorods. Appl Surf Sci 368:456-463. https:// doi.org/10.1016/j.apsusc.2016.01.253

14. Amin G, Asif MH, Zainelabdin A et al (2011) Influence of $\mathrm{pH}$, precursor concentration, growth time, and temperature on the morphology of $\mathrm{ZnO}$ nanostructures grown by the hydrothermal method. J Nanomater 2011:5:5. https://doi.org/10.1155/2011/269692

15. Verrier C, Appert E, Chaix-Pluchery O et al (2017) Effects of the $\mathrm{pH}$ on the formation and doping mechanisms of $\mathrm{ZnO}$ nanowires using aluminum nitrate and ammonia. Inorg Chem 56:13111-13122. https://doi. org/10.1021/acs.inorgchem.7b01916

16. Wei Y, Wu W, Guo Ret al (2010) Wafer-scale high-throughput ordered growth of vertically aligned $\mathrm{ZnO}$ nanowire arrays. Nano Lett 10:3414 3419. https://doi.org/10.1021/nl1014298

17. Volk J, Nagata T, Erdélyi R et al (2009) Highly uniform epitaxial ZnO nanorod arrays for nanopiezotronics. Nanoscale Res Lett 4:699-704. https ://doi.org/10.1007/s11671-009-9302-1

18. Szabó Z, Volk J, Fülöp E et al (2013) Regular ZnO nanopillar arrays by nanosphere photolithography. Photonics Nanostruct Fundam Appl 11:1-7. https://doi.org/10.1016/j.photonics.2012.06.009

19. Szabó Z, Cora I, Horváth Z et al (2018) Hierarchical oxide nanostructures fabricated with atomic layer deposition and hydrothermal growth. Nano Struct Nano Objects 13:100-108. https://doi.org/10.1016/j.nanos 0.2017 .12 .006
20. Sakai D, Nagashima K, Yoshida H et al (2019) Substantial narrowing on the width of "concentration window" of hydrothermal $\mathrm{ZnO}$ nanowires via ammonia addition. Sci Rep 9:1-10. https://doi.org/10.1038/s41598-01950641-y

21. Pham HT, Nguyen TD, Tran DQ, Akabori M (2017) Structural, optical and electrical properties of well-ordered $\mathrm{ZnO}$ nanowires grown on ( $\left.\begin{array}{lll}1 & 1 & 1\end{array}\right)$ oriented $\mathrm{Si}$, GaAs and InP substrates by electrochemical deposition method. Mater Res Express 4:055002. https://doi.org/10.1088/2053-1591/aa6c9a

22. Parize R, Garnier JD, Appert E et al (2018) Effects of polyethylenimine and its molecular weight on the chemical bath deposition of $\mathrm{ZnO}$ nanowires. ACS Omega 3:12457-12464. https://doi.org/10.1021/acsomega.8b01641

23. Alnoor H, Pozina G, Khranovskyy V et al (2016) Influence of ZnO seed layer precursor molar ratio on the density of interface defects in low temperature aqueous chemically synthesized ZnO nanorods/GaN lightemitting diodes. J Appl Phys 119:165702. https://doi.org/10.1063/1.49475 93

24. Fredriksson $\mathrm{H}$, Alaverdyan $\mathrm{Y}$, Dmitriev A et al (2007) Hole-mask colloidal lithography. Adv Mater 19:4297-4302. https://doi.org/10.1002/ adma. 200700680

25. Hanarp P, Sutherland D, Gold J, Kasemo B (1999) Nanostructured model biomaterial surfaces prepared by colloidal lithography. Nanostruct Mater 12:429-432. https://doi.org/10.1016/50965-9773(99)00151-8

26. Cesaria M, Taurino A, Manera MG et al (2019) Gold nanoholes fabricated by colloidal lithography: novel insights into nanofabrication, short-range correlation and optical properties. Nanoscale 11:8416-8432. https://doi. org/10.1039/C8NR09911A

27. Chen K, Thang DD, Ishii S et al (2015) Selective patterned growth of ZnO nanowires/nanosheets and their photoluminescence properties. Opt Mater Express: OME 5:353-360. https://doi.org/10.1364/OME.5.000353

28. Vellekoop MJ, Visser CCO, Sarro PM, Venema A (1990) Compatibility of zinc oxide with silicon IC processing. Sens Actuators A 23:1027-1030. https://doi.org/10.1016/0924-4247(90)87083-U

29. Nian Q, Callahan M, Look D et al (2015) Highly transparent conductive electrode with ultra-low HAZE by grain boundary modification of aqueous solution fabricated alumina-doped zinc oxide nanocrystals. APL Mater 3:062803. https://doi.org/10.1063/1.4915489

30. Kumar M, Kanjilal A, Som T (2013) Effect of grain-boundaries on electrical properties of $\mathrm{n}$-ZnO:Al/p-Si heterojunction diodes. AIP Adv 3:092126. https://doi.org/10.1063/1.4823480

31. Bandyopadhyay S, Paul GK, Roy R et al (2002) Study of structural and electrical properties of grain-boundary modified ZnO films prepared by sol-gel technique. Mater Chem Phys 74:83-91. https://doi.org/10.1016/ S0254-0584(01)00402-3

32. Zhang Z, Meng G, Xu Q et al (2010) Aligned ZnO nanorods with tunable size and field emission on native si substrate achieved via simple electrodeposition. J Phys Chem C 114:189-193. https://doi.org/10.1021/jp908 7223

33. Pea M, Maiolo L, Pilloton R et al (2014) ZnO nanowires strips growth: template reliability and morphology study. Microelectron Eng 121:147-152. https://doi.org/10.1016/j.mee.2014.04.045

34. Watanabe K, Nagata T, Oh S et al (2016) Arbitrary cross-section SEMcathodoluminescence imaging of growth sectors and local carrier concentrations within micro-sampled semiconductor nanorods. Nat Commun 7:10609. https://doi.org/10.1038/ncomms10609

35. Consonni V, Lord AM (2021) Polarity in ZnO nanowires: a critical issue for piezotronic and piezoelectric devices. Nano Energy 83:105789. https:// doi.org/10.1016/j.nanoen.2021.105789

36. Zhou Q, Wen JZ, Zhao P, Anderson WA (2017) Synthesis of verticallyaligned zinc oxide nanowires and their application as a photocatalyst. Nanomaterials 7:9. https://doi.org/10.3390/nano7010009

\section{Publisher's Note}

Springer Nature remains neutral with regard to jurisdictional claims in published maps and institutional affiliations. 


\section{Paper IV}

"Nanopatterned reduced graphene oxide/Al-doped $\mathrm{ZnO}$ seed layer for vertical growth of single $\mathrm{ZnO}$ nanorods on various substrates" has been removed for copyright reasons. For more details about these see:

http://urn.kb.se/resolve?urn=urn:nbn:se:liu:diva-174835 


\section{FACULTY OF SCIENCE AND ENGINEERING}

Linköping Studies in Science and Technology, Dissertation No. 2130

Department of Science and Technology, 2021

Linköping University

SE-581 83 Linköping, Sweden

www.liu.se

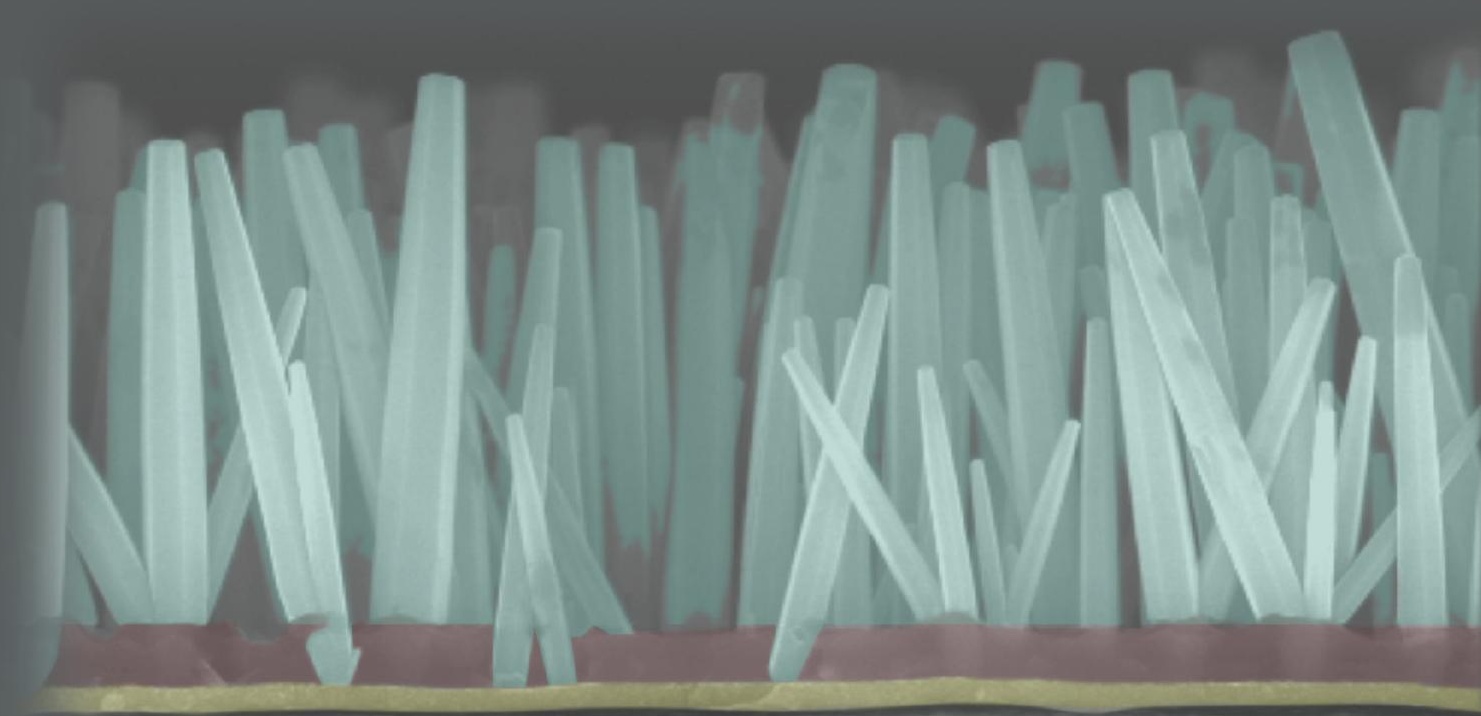

